\title{
Lectures on AKSZ Sigma Models for Physicists
}

\author{
Noriaki IKEDA $\left.\right|^{a}$ \\ Department of Mathematical Sciences, Ritsumeikan University \\ Kusatsu, Shiga 525-8577, Japan
}

January 31, 2017

\begin{abstract}
This is an introductory review of topological field theories (TFTs) called AKSZ sigma models. The AKSZ construction is a mathematical formulation for the construction and analysis of a large class of TFTs, inspired by the Batalin-Vilkovisky formalism of gauge theories. We begin by considering a simple two-dimensional topological field theory and explain the ideas of the AKSZ sigma models. This construction is then generalized and leads to a mathematical formulation of a general topological sigma model. We review the mathematical objects, such as algebroids and supergeometry, that are used in the analysis of general gauge structures. The quantization of the Poisson sigma model is presented as an example of a quantization of an AKSZ sigma model.
\end{abstract}

${ }^{a}$ E-mail: nikeda@se.ritsumei.ac.jp 


\section{Contents}

1 Introduction $\quad 2$

2 Topological Field Theory in Two Dimensions 4

2.1 Two-Dimensional Abelian BF Theory . . . . . . . . . . . . . . . 5

2.2 Deformation of Two-Dimensional Abelian BF Theory . . . . . . . . . . . . 8

2.3 Poisson Sigma Model . . . . . . . . . . . . . . . . . . . 10

2.4 Superfield Formalism . . . . . . . . . . . . . . . . . . 14

3 Abelian BF Theories for $i$-Form Gauge Fields in Higher Dimensions 16

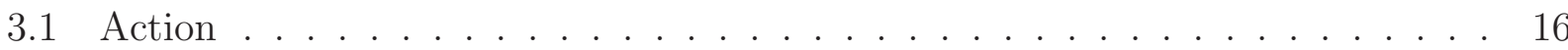

3.2 BV Formalism . . . . . . . . . . . . . . . . . . . 18

3.3 Superfield Formalism . . . . . . . . . . . . . . . . . . . . . . 19

4 QP-manifolds $\quad 21$

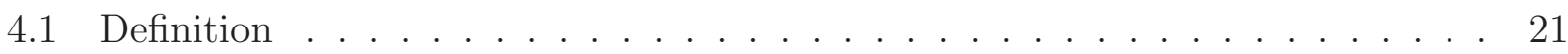

4.2 Notation . . . . . . . . . . . . . . . . . . . 22

5 Examples of QP-Manifolds $\quad 23$

5.1 Lie Algebra and Lie Algebroid as QP-manifold of degree $n$. . . . . . . . 23

5.1 .1 Lie Algebra . . . . . . . . . . . . . . . . 23

5.1 .2 Lie Algebroid . . . . . . . . . . . . . . . . . . . 24

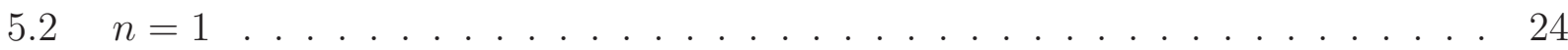

5.2 .1 Poisson Structure . . . . . . . . . . . . . . . . 25

5.2 .2 Complex Structure . . . . . . . . . . . . . . . 25

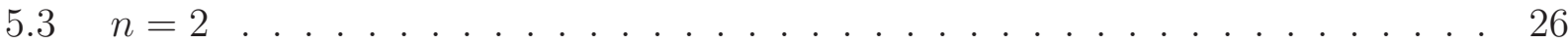

5.3.1 Courant Algebroid ....................... 26

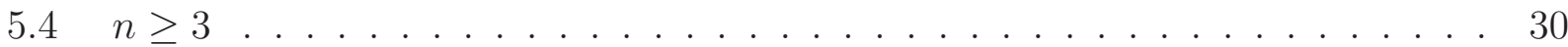

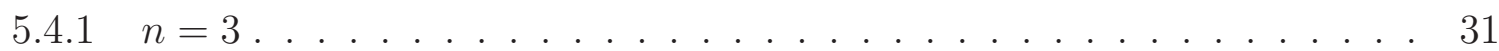

5.4.2 Higher Dorfman Bracket . . . . . . . . . . . . . . . 32

5.4 .3 Nonassociative Example . . . . . . . . . . . . . . . . 33

6 AKSZ Construction of Topological Field Theories 34 
8 AKSZ Sigma Models in Local Coordinates 40

9 Examples of AKSZ Sigma Models 44

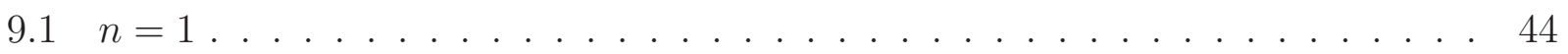

9.1.1 The Poisson Sigma Model . . . . . . . . . . . . . . . . . . . . . . 44

$9.1 .2 \quad$ B-Model . . . . . . . . . . . . . . . . . . . . . . . . 44

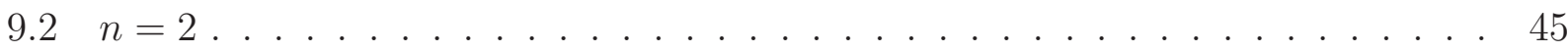

9.2.1 The Courant Sigma Model . . . . . . . . . . . . . . . . . . . . 45

$9.2 .2 \quad$ Chern-Simons Gauge Theory . . . . . . . . . . . . . . . . . . . . . 46

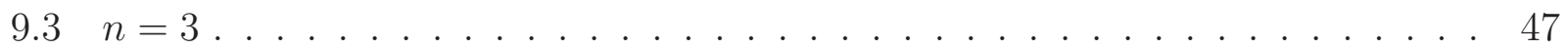

9.3.1 AKSZ Sigma Model in 4 Dimensions . . . . . . . . . . . . . . . 47

9.3.2 Topological Yang-Mills Theory _. . . . . . . . . . . . . . 47

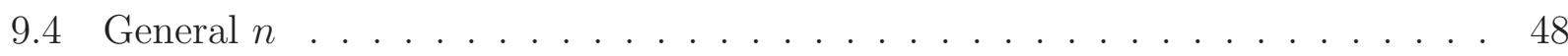

9.4.1 Nonabelian BF Theories in $n+1$ Dimensions $\ldots \ldots \ldots . . \ldots 48$

9.4.2 Nonassociative Topological Field Theory . . . . . . . . . . . . . . . 48

10 AKSZ Sigma Models with Boundary 49

$10.1 n=2$ : WZ-Poisson Sigma Model . . . . . . . . . . . . . . 49

10.2 General Structures of AKSZ Sigma Models with Boundary . . . . . . . . . 53

10.3 Canonical Transformation of Q-structure Function . . . . . . . . . . 55

10.4 From Twist to Boundary Terms . . . . . . . . . . . . . . . . . 56

11 Topological Strings from AKSZ Sigma Models

11.1 A-Model . . . . . . . . . . . . . . . . . . . . . . . 57

11.2 B-Model . . . . . . . . . . . . . . . . . . . . . . . . . . . . . . . . . . . . . . . 5

$\begin{array}{ll}12 \text { Quantization } & 60\end{array}$

12.1 Poisson Sigma Model on a Disc . . . . . . . . . . . . . . . . . . 60 60

12.1.1 Deformation Quantization .................. . . . 60

12.1.2 Path Integrals . . . . . . . . . . . . . . . . . . 61 61

12.1.3 BV Quantization ... . . . . . . . . . . . . . . . 662 
12.1.4 Boundary Conditions . . . . . . . . . . . . . . . . . 63

12.1.5 Propagators . . . . . . . . . . . . . . . . . 65

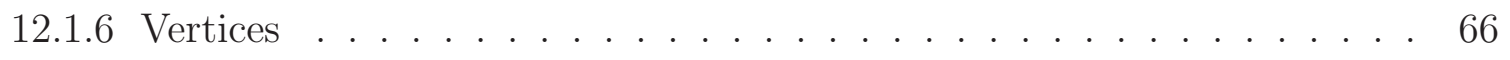

12.1.7 Renormalization of Tadpoles . . . . . . . . . . . . . . 66

12.1.8 Correlation Functions of Observables on the Boundary . . . . . . . . 67

12.1.9 Associativity and Equivalence . . . . . . . . . . . . . . 68

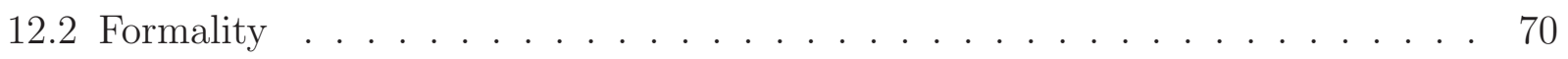

12.2.1 Differential Graded Lie Algebras . . . . . . . . . . . . . . 70

12.2.2 Maurer-Cartan Equations of Poisson Bivector Fields . . . . . . . . . . 70

12.2.3 Hochschild Complex of Polydifferential Operators . . . . . . . . . . 71

12.2.4 Morphisms of Two Differential Graded Lie Algebras . . . . . . . . . . . 72

12.2.5 $L_{\infty}$-Algebras and $L_{\infty}$-Morphisms . . . . . . . . . . . . . . . 73

12.2.6 Correspondence to $n=1$ AKSZ Sigma Model . . . . . . . . . . 75

13 Comments and Future Outlook $\quad 76$

A Appendix: Formulas in Graded Differential Calculus $\quad 78$

A.1 Basic definitions . . . . . . . . . . . . . . . . . . . . . . . 79

A.2 Cartan formulas . . . . . . . . . . . . . . . . . . . . 80

A.3 Differential forms . . . . . . . . . . . . . . . . . . . . . 80

A.3.1 Exterior derivatives . . . . . . . . . . . . . . . . 81

A.4 Graded symplectic form and Poisson bracket . . . . . . . . . . . . . 82

\section{Introduction}

This lecture note will present basics of so-called AKSZ (Alexandrov-Kontsevich-SchwarzZaboronsky) sigma models. Though there are several reviews which present mathematical aspects of AKSZ construction and AKSZ sigma models [100, 42, 121], in this lecture, we will introduce these theories by the physics language and explain mathematical foundations gently. Thus, mathematical rigor will sometimes be sacrificed.

An AKSZ sigma model is a type of topological field theory (TFT). TFT was proposed by Witten [149, 150] as a special version of a quantum field theory. After that, a mathemati- 
cal definition has been provided [6]. Apart from it, this theory has been formulated by the (BRST and) Batalin-Vilkovisky (BV) formalism [13, 132, 133] of gauge theories. The AKSZ construction [5, 35] is a reformulation of a TFT in this direction. Purpose of the latter formulation is to analyze classical and quantum aspects of topological field theories by the action principle and the physical quantization technique, which is fundamental to the formulation of a gauge theory, and to apply them to various physical and mathematical problems.

The AKSZ construction is a powerful formulation since a large class of TFTs are constructed and unified by this construction. These include known TFTs, such as the A-model, the B-model [151], BF theory [68], Chern-Simons theory [149], topological Yang-Mills theory [149], Rozansky-Witten theory [127], the Poisson sigma model [76, 69, 129], the Courant sigma model [72, 66, 124], and Schwarz-type TFTs [132, 133]. Moreover, we find that the AKSZ sigma models contain more TFTs, which, for instance, have the structure of Lie algebroids, Courant algebroids, homotopy Lie algebras, or their higher generalizations.

We start this lecture note by explaining the simplest example to introduce idea of the AKSZ construction, which is the two-dimensional abelian BF theory, First, we express this theory using the BV formalism. Next, deformation theory is used to find the most general consistent interaction term that satisfies physical properties. As a result, we obtain the Poisson sigma model, an important nontrivial two-dimensional topological sigma model of AKSZ type. As another example, we also consider the BV formalism of an abelian BF theory in higher dimensions. From the analysis of these models, we identify the mathematical components of the AKSZ construction, a QP-manifold.

In the next section, we explain the basic mathematical notion, a QP-manifold, a differential graded symplectic manifold. It is a triple consisting of a graded manifold, a graded Poisson structure, and a coboundary operator called homological vector field.

Based on the QP-manifold structure, we construct a sigma model as a map between two graded manifolds, from $\mathcal{X}$ to $\mathcal{M}$, which is the AKSZ construction. We discuss that structures of the target space and gauge symmetries of AKSZ sigma models are derived from this QPmanifold. We analyze the gauge symmetries of general forms of AKSZ sigma models, which are deformations of abelian BF theories, and we will find that the infinitesimal gauge symmetry algebras of these models are not Lie algebras. This analysis leads us to the introduction of Lie algebroids and their generalizations as gauge symmetries of AKSZ sigma models. The 
finite versions of these gauge symmetries corresponding to Lie groups are groupoids. These mathematical objects which are not so familiar to physicists are explained by using local coordinate expressions.

In the last part of this lecture note, two important applications of AKSZ sigma models are discussed. One is the derivation of topological strings. The A-model and the B-model are derived by gauge-fixing AKSZ sigma models in two dimensions [5]. The other application is the deformation quantization on a Poisson manifold. The quantization of the Poisson sigma model on a disc provides a star product formula on the target space [93, 33]. The second application is also a prototype of the quantization of AKSZ sigma models; although such quantizations have been successfully carried out in only a few cases, this example is one such case.

This lecture note is organized as follows. In Section 2, the BV formalism of an abelian BF theory in two dimensions is considered and an interaction term is determined by deformation theory. This theory is reconstructed by the superfield formalism. In Section 3, an abelian $\mathrm{BF}$ theory in higher dimensions is constructed by the BV formalism and reformulated by the superfield formalism. In Section 4, a QP-manifold, which is the mathematical object for the AKSZ construction is defined. In Section 5, important examples are listed. In Section 6, the AKSZ construction is defined and explained. In Section 7, we use deformation theory to obtain general consistent interaction terms for general AKSZ sigma models. In Section 8, we express an AKSZ sigma model in local coordinates. In Section 9, we provide some examples of AKSZ sigma models. In Section 10, we analyze AKSZ sigma models on an open manifold. In Sections 11 and 12, we discuss two important applications, review the derivation of the A-model and the B-model and present a deformation quantization on a Poisson manifold from the quantization of the Poisson sigma model. Section 13 is devoted to discussing related works and areas of future investigation.

\section{Topological Field Theory in Two Dimensions}

We begin by explaining the concept of the AKSZ construction by providing a simple example. We consider an abelian BF theory in two dimensions and discuss its Batalin-Vilkovisky formalism. A consistent interaction term is introduced by using deformation theory. Finally, we 
present a mathematical construction of its interacting theory by using the AKSZ construction.

\subsection{Two-Dimensional Abelian BF Theory}

The simplest topological field theory is a two-dimensional abelian BF theory. Let $\Sigma$ be a manifold in two dimensions with a local coordinate $\sigma^{\mu}(\mu=0,1)$ and suppose that $\Sigma$ has no boundary. Here, we will take the Euclidean signature.

Let $A_{\mu i}(\sigma)$ be a gauge field and let $\phi^{i}(\sigma)$ be a scalar field, where $i=1,2, \cdots, d$ is an index on $d$-dimensional target space. The action is as follows:

$$
S_{A}=-\frac{1}{2} \int_{\Sigma} d^{2} \sigma \epsilon^{\mu \nu} F_{0 \mu \nu i} \phi^{i}=\int_{\Sigma} d^{2} \sigma \epsilon^{\mu \nu} A_{\mu i} \partial_{\nu} \phi^{i},
$$

where $F_{0 \mu \nu i}=\partial_{\mu} A_{\nu i}-\partial_{\nu} A_{\mu i}$ is the field strength. Note that the boundary integral vanishes. The gauge symmetry of this theory is $U(1)$ :

$$
\delta_{0} A_{\mu i}=\partial_{\mu} \epsilon_{i}, \quad \delta_{0} \phi^{i}=0,
$$

where $\epsilon_{i}(\sigma)$ is a gauge parameter.

Let us consider the following problem. We add terms to $S_{A}$ and deform the gauge symmetry $\delta_{0}$ as follows:

$$
\begin{aligned}
& S=S_{A}+S_{I}, \\
& \delta=\delta_{0}+\delta_{1} .
\end{aligned}
$$

We search for the consistent $S$ and $\delta$. The new action $S$ and the new modified gauge symmetry $\delta$ must satisfy the following two consistency conditions: The action is gauge invariant, that is, $\delta S=0$; and the gauge symmetry algebra is closed, at least under the equations of motion, $\left[\delta_{\epsilon}, \delta_{\epsilon^{\prime}}\right] \approx \delta_{\left[\epsilon, \epsilon^{\prime}\right]}$. Note that $\delta S=0$ must be satisfied without the equations of motion, but it is sufficient to satisfy the closedness condition for the gauge algebra, $\left[\delta_{\epsilon}, \delta_{\epsilon^{\prime}}\right]=\delta_{\left[\epsilon, \epsilon^{\prime}\right]}$ along the orbit of the equations of motion.

In order to construct a consistent field theory, physical conditions are imposed on $S$ : It is required to be diffeomorphism invariant, local and unitary. Two actions are equivalent if they become classically the same action when there is local replacement of the fundamental fields. That is, if two actions coincide, $\tilde{S}(\tilde{\Phi})=S(\Phi)$, under a local redefinition of the fields, $\tilde{\Phi}=f(\Phi)$, then they are equivalent. Moreover, we regard two theories as equivalent if they 
have the same gauge symmetry, i.e. $\delta_{1}=0$. As required by a local field theory, we have a Lagrangian $\mathcal{L}$ such that $S=\int_{\Sigma} d^{2} \sigma \mathcal{L}$, where $\mathcal{L}$ is a function of the local fields. We assume that $\mathcal{L}$ is at most a polynomial with respect to a gauge field $A_{\mu i}$.

The problem is to determine the most general $S_{I}$ under the assumptions discussed above. In order to unify the conditions $\delta S=0$ and $\left[\delta_{\epsilon}, \delta_{\epsilon^{\prime}}\right]=\delta_{\left[\epsilon, \epsilon^{\prime}\right]}+$ (equations of motion), we use the BV formalism to formulate the theory. This is the most general method for obtaining a consistent gauge theory.

Let us apply the BV formalism to this abelian BF theory [63, 52]. First, a gauge parameter $\epsilon_{i}$ is replaced by the Faddeev-Popov (FP) ghost $c_{i}$, which is a Grassmann-odd scalar field. $b$ The ghost numbers of the fields $\Phi \in\left\{A_{\mu i}, \phi^{i}, c_{i}\right\}$, gh $\Phi$, are defined as $\operatorname{gh} A_{\mu i}=\operatorname{gh} \phi^{i}=0$ and $\operatorname{gh} c_{i}=1$. The gauge transformation $\delta_{0}$ is changed to a BRST transformation such that $\delta_{0}^{2}=0$ by replacement of the gauge parameter with the FP ghost. This condition imposes $\delta_{0} c_{i}=0$.

For each of the fields $\Phi$, we introduce an antifield $\Phi^{*} \in\left\{A^{* \mu i}, \phi_{i}^{*}, c^{* i}\right\}$. Compared to the corresponding field, the antifield has the opposite Grassmann properties but the same spin. The ghost numbers of the antifields are defined by the equation gh $\Phi+\operatorname{gh} \Phi^{*}=-1$. For ghost number $-1, A^{* \mu i}$ is a vector and $\phi_{i}^{*}$ is a scalar field. $c^{* i}$ is a scalar field of ghost number -2 .

Table 1: Ghost number and form degree of fields and antifields

\begin{tabular}{|c|c|c|c|c|}
\hline form degree ghost number & -2 & -1 & 0 & 1 \\
\hline $\begin{array}{l}0 \\
1\end{array}$ & $c^{* i}$ & $\begin{array}{r}\phi_{i}^{*} \\
A^{* \mu i}\end{array}$ & $\begin{array}{r}\phi^{i} \\
A_{\mu i}\end{array}$ & $c_{i}$ \\
\hline
\end{tabular}

Next, an odd Poisson bracket, called the antibracket, is introduced as $\left\{\Phi(\sigma), \Phi^{*}\left(\sigma^{\prime}\right)\right\}=$ $-\left\{\Phi^{*}\left(\sigma^{\prime}\right), \Phi(\sigma)\right\}=\delta^{2}\left(\sigma-\sigma^{\prime}\right)$. It is written as

$$
\{F, G\} \equiv \sum_{\Phi} \int_{\Sigma} d^{2} \sigma\left(F \frac{\overleftarrow{\partial}}{\partial \Phi(\sigma)} \frac{\vec{\partial}}{\partial \Phi^{*}\left(\sigma^{\prime}\right)} G-F \frac{\overleftarrow{\partial}}{\partial \Phi^{*}(\sigma)} \frac{\vec{\partial}}{\partial \Phi\left(\sigma^{\prime}\right)} G\right) \delta^{2}\left(\sigma-\sigma^{\prime}\right)
$$

where the differentiation is the functional differentiation, and $F \frac{\overleftarrow{\partial}}{\partial \Phi(\sigma)}=(-1)^{(\operatorname{gh} F-\operatorname{gh} \Phi)(\operatorname{gh} \Phi)} \frac{\partial F}{\partial \Phi(\sigma)}$ denotes right derivative and $\frac{\vec{\partial}}{\partial \Phi^{*}\left(\sigma^{\prime}\right)} F=\frac{\partial F}{\partial \Phi^{*}\left(\sigma^{\prime}\right)}$ denotes left derivative. The antibracket is

\footnotetext{
${ }^{b}$ This is the Faddeev-Popov method of the quantization of a gauge theory.
} 
graded symmetric and it satisfies the graded Leibniz rule and the graded Jacobi identity:

$$
\begin{aligned}
& \{F, G\}=-(-1)^{(\operatorname{gh} F+1)(\operatorname{gh} G+1)}\{G, F\}, \\
& \{F, G H\}=\{F, G\} H+(-1)^{(\operatorname{gh} F+1) \operatorname{gh} G} G\{F, H\}, \\
& \{F G, H\}=F\{G, H\}+(-1)^{\operatorname{gh} G(\operatorname{gh} H+1)}\{F, H\} G, \\
& (-1)^{(\operatorname{gh} F+1)(\operatorname{gh} H+1)}\{F,\{G, H\}\}+\text { cyclic permutations }=0,
\end{aligned}
$$

where $F, G$, and $H$ are functions of $\Phi$ and $\Phi^{*}$.

Finally, the BV action $S^{(0)}$ is constructed as follows:

$$
S^{(0)}=S_{A}+(-1)^{\operatorname{gh} \Phi} \int_{\Sigma} \Phi^{*} \delta_{0} \Phi+O\left(\Phi^{* 2}\right),
$$

where $O\left(\Phi^{* 2}\right)$ is determined order by order to satisfy $\left\{S^{(0)}, S^{(0)}\right\}=0$, which is called the classical master equation. In the abelian BF theory, the BV action is defined by adding ghost terms as follows:

$$
S^{(0)}=\int_{\Sigma} d^{2} \sigma \epsilon^{\mu \nu} A_{\mu i} \partial_{\nu} \phi^{i}+\int_{\Sigma} d^{2} \sigma A^{* \nu i} \partial_{\nu} c_{i},
$$

and $O\left(\Phi^{* 2}\right)=0$. It is easily confirmed that $S^{(0)}$ satisfies the classical master equation.

The BRST transformation in the BV formalism is

$$
\delta_{0} F\left[\Phi, \Phi^{*}\right]=\left\{S^{(0)}, F\left[\Phi, \Phi^{*}\right]\right\},
$$

which coincides with the gauge transformation on fields $\Phi$. The explicit BRST transformations are

$$
\begin{aligned}
& \delta_{0} A_{\mu i}=\partial_{\mu} c_{i}, \quad \delta_{0} A^{* \mu i}=\epsilon^{\mu \nu} \partial_{\nu} \phi^{i}, \\
& \delta_{0} \phi^{*}{ }_{i}=\epsilon^{\mu \nu} \partial_{\mu} A_{\nu i}, \quad \delta_{0} c^{* i}=-\partial_{\mu} A^{* \mu i},
\end{aligned}
$$

and zero for all other fields. The classical master equation, $\left\{S^{(0)}, S^{(0)}\right\}=0$, guarantees two consistency conditions: gauge invariance of the action and closure of the gauge algebra. Gauge invariance of the action is proved as $\delta_{0} S^{(0)}=\left\{S^{(0)}, S^{(0)}\right\}=0$. Closure of the gauge symmetry algebra is proved as $\delta_{0}^{2} F=\left\{S^{(0)},\left\{S^{(0)}, F\right\}\right\}=\frac{1}{2}\left\{\left\{S^{(0)}, S^{(0)}\right\}, F\right\}=0$ by using the Jacobi identity. 


\subsection{Deformation of Two-Dimensional Abelian BF Theory}

The deformation theory of a gauge theory is a systematic method for obtaining a new gauge theory from a known one [11, 8, 62. Deformation theory within the BV formalism locally determines all possible nontrivial consistent interaction terms $S_{I}$.

We consider the deformation of a $\mathrm{BV}$ action $S^{(0)}$ to $S$ as follows:

$$
S=S^{(0)}+g S^{(1)}+g^{2} S^{(2)}+\cdots,
$$

under the fixed antibracket $\{-,-\}$, where $g$ is a deformation parameter. Consistency requires the classical master equation, $\{S, S\}=0$, on the resulting action $S$. Moreover, we require an equivalence relation, that is, $S^{\prime}$ is equivalent to $S$ if and only if $S^{\prime}=S+\{S, T\}$, where $T$ is the integral of a local term in the fields and antifields. This condition corresponds to the physical equivalence discussed in the previous subsection. $S^{(n)}(n=1,2, \cdots)$ is determined order by order by solving the expansions of the classical master equation with respect to $g^{n}$. Invariance, locality and unitarity (the physical conditions discussed in the previous subsection) are required in order for the resulting action to be physically consistent. From these requirements, $S$ is diffeomorphism invariant on $\Sigma$, it is the integral of a local function (Lagrangian) $\mathcal{L}$ on $\Sigma$, and it has ghost number 0 .

We substitute equation (2.3) into the classical master equation $\{S, S\}=0$. At order $g^{0}$, we obtain $\left\{S^{(0)}, S^{(0)}\right\}=0$. This equation is already satisfied, since it is the classical master equation of the abelian BF theory.

At order $g^{1}$, we obtain

$$
\left\{S^{(0)}, S^{(1)}\right\}=\delta_{0} S^{(1)}=0 .
$$

From the assumption of locality, $S^{(1)}$ is an integral of a 2 -form $\mathcal{L}^{(1)}$ such that $S^{(1)}=\int_{\Sigma} \mathcal{L}^{(1)}$. Thus, equation (2.4) requires that $\delta_{0} \mathcal{L}^{(1)}$ be a total derivative. Then, the following equations are obtained by repeating the same arguments for the descent terms:

$$
\begin{aligned}
& \delta_{0} \mathcal{L}^{(1)}+d a_{1}=0, \\
& \delta_{0} a_{1}+d a_{0}=0, \\
& \delta_{0} a_{0}=0,
\end{aligned}
$$


where $a_{1}$ is a 1 -form of ghost number $1, a_{0}$ is a 0 -form of ghost number 2 . $a_{0}$ can be determined as

$$
a_{0}=-\frac{1}{2} f^{i j}(\phi) c_{i} c_{j}
$$

up to $\delta_{0}$ exact terms. Here, $f^{i j}(\phi)$ is an arbitrary function of $\phi$ such that $f^{i j}(\phi)=-f^{j i}(\phi)$. Note that terms including the metric on $\Sigma$ and terms including differentials $\partial_{\mu}$ can be dropped, since those terms are $\delta_{0}$ exact up to total derivatives. If we solve the descent equation, then

$$
a_{1}=f^{i j} A_{i} c_{j}-\frac{1}{2} \frac{\partial f^{i j}}{\partial \phi^{k}} A^{+k} c_{i} c_{j},
$$

up to BRST exact terms, and finally $\mathcal{L}^{(1)}$ is uniquely determined as

$$
\begin{aligned}
\mathcal{L}^{(1)}= & \frac{1}{2} f^{i j}\left(A_{i} A_{j}-2 \phi_{i}^{+} c_{j}\right)+\frac{\partial f^{i j}}{\partial \phi^{k}}\left(\frac{1}{2} c^{+k} c_{i} c_{j}+A^{+k} A_{i} c_{j}\right) \\
& -\frac{1}{4} \frac{\partial^{2} f^{i j}}{\partial \phi^{k} \partial \phi^{l}} A^{+k} A^{+l} c_{i} c_{j}
\end{aligned}
$$

up to BRST exact terms [84]. Here, $A_{i} \equiv d \sigma^{\mu} A_{\mu i}, A^{+i} \equiv d \sigma^{\mu} \epsilon_{\mu \nu} A^{* \nu i}, \phi_{i}^{+} \equiv * \phi_{i}^{*}$, and $c^{+i} \equiv * c^{* i}$, where $*$ is the Hodge star on $\Sigma$. From the definition of the BRST transformations, we have ${ }^{c}$

$$
\begin{aligned}
& \delta_{0} A_{i}=d c_{i}, \quad \delta_{0} \phi^{+}{ }_{i}=d A_{i}, \\
& \delta_{0} A^{+i}=-d \phi^{i}, \quad \delta_{0} c^{+i}=d A^{+i} .
\end{aligned}
$$

At order $g^{2}$, the master equation is $\left\{S^{(1)}, S^{(1)}\right\}+2\left\{S^{(0)}, S^{(2)}\right\}=0$. From the assumption of locality, $S^{(2)}$ is an integral of a local function $\mathcal{L}^{(2)}$ of fields and antifields. Since $\delta_{0}(\Psi) \propto \partial_{\mu}(*)$ for all the fields and antifields up to $\delta_{0}$ exact terms, $\left\{S^{(0)}, S^{(2)}\right\}=\int d \mathcal{L}^{(2)}=0$ if there is no boundary term. The condition $\left\{S^{(0)}, S^{(2)}\right\}=0$ for $S^{(2)}$ is the same as the condition for $S^{(1)}$. This means that if $S^{(1)}$ is redefined as $S^{(1) \prime}=S^{(1)}+g S^{(2)}, S^{(2)}$ can be absorbed into $S^{(1)}$. d

Continuing this procedure order by order, we obtain all the consistency conditions:

$$
\begin{aligned}
& \left\{S^{(1)}, S^{(1)}\right\}=0, \\
& S^{(n)}=0, \quad(n=2,3, \cdots) .
\end{aligned}
$$

${ }^{c}$ In the Lorentzian signature, the transformations of $A^{+}$and $c^{+}$have opposite sign.

${ }^{d}$ This is because $S_{0}$ is the action of the abelian BF theory. This equation will not be satisfied for a different $S_{0}$. 
Substituting equation (2.5) into $\left\{S^{(1)}, S^{(1)}\right\}=0$, we obtain the following condition on $f^{i j}(\phi)$ :

$$
\frac{\partial f^{i j}}{\partial \phi^{m}}(\phi) f^{m k}(\phi)+\frac{\partial f^{j k}}{\partial \phi^{m}}(\phi) f^{m i}(\phi)+\frac{\partial f^{k i}}{\partial \phi^{m}}(\phi) f^{m j}(\phi)=0 .
$$

We have found the general solution for the deformation of the two-dimensional abelian BF theory [84]. The complete BV action is as follows:

$$
\begin{aligned}
S= & S^{(0)}+g S^{(1)} \\
= & \int_{\Sigma}\left(A_{i} d \phi^{i}+A^{+i} d c_{i}+g\left(\frac{1}{2} f^{i j}\left(A_{i} A_{j}-2 \phi_{i}^{+} c_{j}\right)\right.\right. \\
& \left.\left.\quad+\frac{\partial f^{i j}}{\partial \phi^{k}}\left(\frac{1}{2} c^{+k} c_{i} c_{j}+A^{+k} A_{i} c_{j}\right)-\frac{1}{4} \frac{\partial^{2} f^{i j}}{\partial \phi^{k} \partial \phi^{l}} A^{+k} A^{+l} c_{i} c_{j}\right)\right) .
\end{aligned}
$$

Here, $f^{i j}(\phi)$ satisfies identity (2.7). If we set $\Phi^{*}=0$, we have the following non-BV action:

$$
\begin{aligned}
S & =\int_{\Sigma} d^{2} \sigma\left(\epsilon^{\mu \nu} A_{\mu i} \partial_{\nu} \phi^{i}+\frac{1}{2} \epsilon^{\mu \nu} f^{i j}(\phi) A_{\mu i} A_{\nu j}\right) \\
& =\int_{\Sigma}\left(A_{i} d \phi^{i}+\frac{1}{2} f^{i j}(\phi) A_{i} A_{j}\right),
\end{aligned}
$$

where $g$ is absorbed by redefinition of $f$. This action is called the Poisson sigma model or nonlinear gauge theory in two dimensions. [76, 69, 128, 129]

Theorem 2.1 The deformation of a two-dimensional abelian BF theory is the Poisson sigma model. 84

This model is considered to be the simplest nontrivial AKSZ sigma model.

\subsection{Poisson Sigma Model}

In this subsection, we list the properties of the Poisson sigma model (2.9).

In special cases, the theory reduces to well-known theories. If $f^{i j}(\phi)=0$, then the theory reduces to the abelian $\mathrm{BF}$ theory:

$$
S_{A}=\int_{\Sigma} d^{2} \sigma \epsilon^{\mu \nu} A_{\mu i} \partial_{\nu} \phi^{i}=\frac{1}{2} \int_{\Sigma} d^{2} \sigma \epsilon^{\mu \nu} \phi^{i} F_{0 \mu \nu i}
$$

If $f^{i j}(\phi)$ is a linear function, $f^{i j}(\phi)=f^{i j}{ }_{k} \phi^{k}$, equation (2.7) is equivalent to the Jacobi identity of the structure constants $f^{i j}{ }_{k}$ of a Lie algebra. The resulting theory is a nonabelian 
BF theory:

$$
S_{N A}=\int_{\Sigma} d^{2} \sigma\left(\epsilon^{\mu \nu} A_{\mu i} \partial_{\nu} \phi^{i}+\frac{1}{2} \epsilon^{\mu \nu} f^{i j} \phi^{k} A_{\mu i} A_{\nu j}\right)=\int_{\Sigma} d^{2} \sigma \epsilon^{\mu \nu} \phi^{i} F_{\mu \nu i}
$$

where $F_{\mu \nu i}=\partial_{\mu} A_{\nu i}-\partial_{\nu} A_{\mu i}+f^{j k}{ }_{i} A_{\mu j} A_{\nu k}$, and this action has the following gauge symmetry:

$$
\delta \phi^{i}=-f^{i j}{ }_{k} \phi^{k} \epsilon_{j}, \quad \delta A_{\mu i}=\partial_{\mu} \epsilon_{i}+\frac{1}{2} f^{j k}{ }_{i} A_{\mu j} \epsilon_{k}
$$

Next, we analyze the symmetry of the Poisson sigma model. The Poisson sigma model has the following gauge symmetry:

$$
\begin{aligned}
& \delta \phi^{i}=-f^{i j}(\phi) \epsilon_{j} \\
& \delta A_{\mu i}=\partial_{\mu} \epsilon_{i}+\frac{1}{2} \frac{\partial f^{j k}(\phi)}{\partial \phi^{i}} A_{\mu j} \epsilon_{k}
\end{aligned}
$$

under the condition given by equation (2.7). In fact, we can directly prove that the requirement $\delta S=0$ under the gauge transformation (2.10) is equivalent to equation (2.7). In the Hamiltonian formalism, the constraints are

$$
G^{i}=\partial_{1} \phi^{i}+f^{i j}(\phi) A_{1 j}
$$

which satisfy the algebra defined by the following Poisson bracket:

$$
\left\{G^{i}(\sigma), G^{j}\left(\sigma^{\prime}\right)\right\}_{P B}=-\frac{\partial f^{i j}}{\partial \phi^{k}} G^{k}(\sigma) \delta\left(\sigma-\sigma^{\prime}\right) .
$$

We can also derive the gauge transformation (2.10) generated by the charge constructed from the constraints $G^{i}(\sigma)$. The gauge algebra has the following form:

$$
\begin{aligned}
& {\left[\delta\left(\epsilon_{1}\right), \delta\left(\epsilon_{2}\right)\right] \phi^{i}=\delta\left(\epsilon_{3}\right) \phi^{i}} \\
& {\left[\delta\left(\epsilon_{1}\right), \delta\left(\epsilon_{2}\right)\right] A_{\mu i}=\delta\left(\epsilon_{3}\right) A_{\mu i}+\epsilon_{1 j} \epsilon_{2 k} \frac{\partial f^{j k}}{\partial \phi^{i} \partial \phi^{l}}(\phi) \epsilon_{\mu \nu} \frac{\delta S}{\delta A_{\nu l}}}
\end{aligned}
$$

where $\epsilon_{1}$ and $\epsilon_{2}$ are gauge parameters, and $\epsilon_{3 i}=\frac{\partial f^{j k}}{\partial \phi^{i}}(\phi) \epsilon_{1 j} \epsilon_{2 k}$. Equation (2.11) for $A_{\mu i}$ shows that the gauge algebra is open. Therefore, this theory cannot be quantized by the BRST formalism and it requires the BV formalism.

This model is a sigma model from a two-dimensional manifold $\Sigma$ to a target space $M$, based on a map $\phi: \Sigma \longrightarrow M$. If equation (2.7) is satisfied on $f^{i j}(\phi)$, then $\{F(\phi), G(\phi)\}_{P B} \equiv$ 
$f^{i j}(\phi) \frac{\partial F}{\partial \phi^{i}} \frac{\partial G}{\partial \phi^{j}}$ defines a Poisson bracket on a target space $M$, since equation (2.7) is the Jacobi identity of this Poisson bracket. $e^{e}$

Conversely, assume that the Poisson bracket on $M$ is given by $\{F(\phi), G(\phi)\}_{P B}=f^{i j}(\phi) \frac{\partial F}{\partial \phi^{2}} \frac{\partial G}{\partial \phi^{j}}$. Then, equation (2.7) is derived from the Jacobi identity and the action given in equation (2.9), which is constructed by this $f^{i j}(\phi)$, is gauge invariant. From this property, the action $S$ is called the Poisson sigma model.

The algebraic structure of the gauge algebra is not a Lie algebra but a Lie algebroid over the cotangent bundle $T^{*} M$. [103]

Definition 2.2 A Lie algebroid over a manifold $M$ is a vector bundle $E \rightarrow M$ with a Lie algebra structure on the space of the sections $\Gamma(E)$ defined by the bracket $\left[e_{1}, e_{2}\right]$, for $e_{1}, e_{2} \in$ $\Gamma(E)$ and a bundle map (the anchor) $\rho: E \rightarrow T M$ satisfying the following properties:

$$
\begin{aligned}
& 1,\left[\rho\left(e_{1}\right), \rho\left(e_{2}\right)\right]=\rho\left(\left[e_{1}, e_{2}\right]\right), \\
& 2,\left[e_{1}, F e_{2}\right]=F\left[e_{1}, e_{2}\right]+\left(\rho\left(e_{1}\right) F\right) e_{2},
\end{aligned}
$$

where $e_{1}, e_{2} \in \Gamma(E), F \in C^{\infty}(M)$ and the bracket $[-,-]$ on the r.h.s. of equation (2.12) is the Lie bracket on the vector fields.

Let us consider the expressions of a Lie algebroid in local coordinates. Let $x^{i}$ be a local coordinate on a base manifold $M$, and let $e_{a}$ be a local basis on the fiber of $E$. The two operations of a Lie algebroid are expressed as

$$
\rho\left(e_{a}\right) F(x)=\rho_{a}^{i}(x) \frac{\partial F(x)}{\partial x^{i}}, \quad\left[e_{a}, e_{b}\right]=f_{a b}^{c}(x) e_{c}
$$

where $i, j, \cdots$ are indices on $M, a, b, \cdots$ are indices of the fiber of the vector bundle $E$, and $\rho_{a}^{i}(x)$ and $f_{a b}^{c}(x)$ are local functions. Then, equations (2.12) and (2.13) are written as

$$
\begin{aligned}
& \rho^{m}{ }_{a} \frac{\partial \rho_{b}^{i}}{\partial \phi^{m}}-\rho^{m}{ }_{b} \frac{\partial \rho^{i}{ }_{a}}{\partial \phi^{m}}+\rho_{c}^{i} f^{c}{ }_{a b}=0, \\
& \rho^{m}{ }_{[a} \frac{\partial f_{b c]}^{d}}{\partial \phi^{m}}+f^{d}{ }_{e[a} f_{b c]}^{e}=0 .
\end{aligned}
$$

Here, we use the notation $f^{d}{ }_{e[a} f_{b c]}^{e}=f_{e a}^{d} f_{b c}^{e}+f_{e b}^{d} f_{c a}^{e}+f_{e c}^{d} f_{a b}^{e}$. For the cotangent bundle $E=T^{*} M$, the indices on the fiber $a, b, \cdots$ run over the same range as the indices $i, j, \cdots$. We ${ }^{e}$ In the notation used in this paper, $\{-,-\}$ is the BV antibracket, and $\{-,-\}_{P B}$ is the usual Poisson bracket. 
can take $\rho^{i j}(\phi)=f^{i j}(\phi)$ and $f_{i}^{j k}(\phi)=\frac{\partial f^{j k}}{\partial \phi^{i}}(\phi)$. Substituting these equations into equation (2.15), we obtain the Jacobi identity (2.7). This special Lie algebroid is called the Poisson Lie algebroid.

The action given by equation (2.9) is unitary, and the fields have no physical degrees of freedom, which can be shown by analyzing it using the constraints in the Hamiltonian analysis or by counting the gauge symmetries in the Lagrangian analysis. The partition function does not depend on the metrics on $\Sigma$ and $M$. That is, the Poisson sigma model is a topological field theory.

In the remaining part of this subsection, we list known applications of the Poisson sigma model.

1. We consider two-dimensional gravity theory as a nontrivial example of a Poisson sigma model [76, 69, 129]. Consider a target manifold $M$ in three dimensions. Let the target space indices be $i=0,1,2$ and $\bar{i}=0,1$. Let us denote $A_{\mu i}=\left(e_{\mu \bar{i}}, \omega_{\mu}\right)$ and $\phi^{i}=\left(\phi^{\bar{i}}, \varphi\right)$. We can take $f^{i j}(\phi)$ as

$$
f^{\bar{i} \bar{j}}\left(\phi^{i}\right)=-\epsilon^{\bar{i} \bar{j}} V(\varphi), \quad f^{2 \bar{i}}\left(\phi^{i}\right)=-f^{\bar{i} 2}=\epsilon^{\bar{i} \bar{j}} \phi_{\bar{j}}, \quad f^{22}\left(\phi^{i}\right)=0 .
$$

Equation (2.16) satisfies equation (2.7), and the action given by equation (2.9) reduces to

$$
S=\int_{\Sigma} \sqrt{-g}\left(\frac{1}{2} \varphi R-V(\varphi)\right)-\phi_{\bar{i}} T^{\bar{i}},
$$

where $g$ is the determinant of the metric $g_{\mu \nu}=\eta^{\bar{i} \bar{j}} e_{\mu \bar{i}} e_{\mu \bar{j}}$ on $\Sigma, R$ is the scalar curvature, and $T^{\bar{i}}$ is the torsion. Here, $e_{\mu \bar{i}}$ is identified with the zweibein, and $\omega_{\mu}^{\bar{i} \bar{j}}=\omega_{\mu} \epsilon^{\bar{i} \bar{j}}$ is the spin connection. This action is the gauge theoretic formalism of a gravitational theory with a dilaton scalar field $\varphi$.

2. Let $G$ be a Lie group. The Poisson sigma model on the target space $T^{*} G$ reduces to the $G / G$ gauged Wess-Zumino-Witten (WZW) model, when $A_{\mu i}$ is properly gauge fixed. [4]

3. If $f^{i j}$ is invertible as an antisymmetric matrix, then $f_{i j}^{-1}$ defines a symplectic form on $M$. Then, $A_{\mu i}$ can be integrated out, and the action (2.9) becomes the so-called A-model,

$$
S=\frac{1}{2} \int_{\Sigma} d^{2} \sigma \epsilon^{\mu \nu} f^{-1}{ }_{i j}(\phi) \partial_{\mu} \phi^{i} \partial_{\nu} \phi^{j},
$$


in which the integrand is the pullback of the symplectic structure on $M$. If $M$ is a complex manifold, the B-model can also be derived from the Poisson sigma model. [5]

4. A Poisson structure can be constructed from a classical r-matrix. A sigma model in two dimensions with a classical r-matrix can be constructed as a special case of the Poisson sigma model [49, 26] which has a Poisson-Lie structure.

5. The Poisson sigma model is generalized by introducing the Wess-Zumino term $\int_{X_{3}} \frac{1}{3 !} H_{i j k}(\phi) d \phi^{i} \wedge$ $d \phi^{j} \wedge d \phi^{k}:$

$$
S=\int_{\Sigma} A_{i} d \phi^{i}+\frac{1}{2} f^{i j}(\phi) A_{i} A_{j}+\int_{X_{3}} \frac{1}{3 !} H_{i j k}(\phi) d \phi^{i} \wedge d \phi^{j} \wedge d \phi^{k}
$$

where $X_{3}$ is a manifold in three dimensions such that $\partial X_{3}=\Sigma$, and $H(\phi)=\frac{1}{3 !} H_{i j k}(\phi) d \phi^{i} \wedge$ $d \phi^{j} \wedge d \phi^{k}$ is the pullback of a closed 3-form on $M$. This action is called the WZ-Poisson sigma model or the twisted Poisson sigma model. [91]

6. Quantization of the Poisson sigma model derives a deformation quantization on a target Poisson manifold. The open string tree amplitudes of the boundary observables of the Poisson sigma model on a disc coincide with the deformation quantization formulas on the Poisson manifold $M$ obtained by Kontsevich. [33] This corresponds to the large B-field limit in open string theory. [135]

\subsection{Superfield Formalism}

From this point onward, we set $g=1$ or equivalently, we absorb $g$ into $f^{i j}(\phi)$. The BV action of the Poisson sigma model (2.8) is simplified by introducing supercoordinates. [33] Let us introduce a Grassmann-odd supercoordinate $\theta^{\mu}(\mu=0,1)$. It is not a spinor but a vector and carries a ghost number of 1 .

Superfields are introduced by combining fields and antifields with $\theta^{\mu}$, as follows:

$$
\begin{aligned}
& \phi^{i}(\sigma, \theta) \equiv \phi^{i}+\theta^{\mu} A_{\mu}^{+i}+\frac{1}{2} \theta^{\mu} \theta^{\nu} c_{\mu \nu}^{+i}=\phi^{i}+A^{+i}+c^{+i}, \\
& \boldsymbol{A}_{i}(\sigma, \theta) \equiv-c_{i}+\theta^{\mu} A_{\mu i}+\frac{1}{2} \theta^{\mu} \theta^{\nu} \phi_{\mu \nu i}^{+}=-c_{i}+A_{i}+\phi_{i}^{+},
\end{aligned}
$$


where each term in the superfield has the same ghost number $\mid f$ Note that in this subsection, the component superfields are assigned the same notation as in the nonsuperfield formalism and $d \sigma^{\mu}$ in the differential form expression of each field is replaced by $\theta^{\mu}$ in equation (2.18). The ghost number is called the degree, $|\boldsymbol{\Phi}|$, in the AKSZ formalism $\mid$ The degree of $\boldsymbol{\phi}$ is zero, and that of $\boldsymbol{A}$ is one. The original fields $\phi^{i}$ and $A_{\mu i}$ appear in $|\phi|$-th order of $\theta$ and $|\boldsymbol{A}|$-th order of $\theta$ components in the superfields, respectively.

With this notation, the BV action of equation (2.8) is summarized as the superintegral of superfields as

$$
S=\int_{T[1] \Sigma} d^{2} \sigma d^{2} \theta\left(\boldsymbol{A}_{i} \boldsymbol{d} \phi^{i}+\frac{1}{2} f^{i j}(\boldsymbol{\phi}) \boldsymbol{A}_{i} \boldsymbol{A}_{j}\right)
$$

where $\boldsymbol{d} \equiv \theta^{\mu} \partial_{\mu}$ is the superderivative and $T[1] \Sigma$ is a supermanifold, which has local coordinates $\left(\sigma^{\mu}, \theta^{\mu}\right)$. The degree of $S$ is zero, $|S|=0$. If we integrate by $d^{2} \theta$, then equation (2.19) reduces to equation (2.8).

The antibrackets of component fields given in (2.1) are combined into a compact form by using the superantibracket as

$$
\{F, G\} \equiv \int_{T[1] \Sigma} d^{2} \sigma d^{2} \theta\left(F \frac{\overleftarrow{\partial}}{\partial \boldsymbol{\phi}^{i}} \frac{\vec{\partial}}{\partial \boldsymbol{A}_{i}} G-F \frac{\overleftarrow{\partial}}{\partial \boldsymbol{A}_{i}} \frac{\vec{\partial}}{\partial \boldsymbol{\phi}^{i}} G\right) \delta^{2}\left(\sigma-\sigma^{\prime}\right) \delta^{2}\left(\theta-\theta^{\prime}\right)
$$

where $F$ and $G$ are functionals of superfields. The classical master equations can be replaced by the super-classical master equation, $\{S, S\}=0$, where the bracket is the super-antibracket. The BRST transformation on a superfield $\boldsymbol{\Phi}=\Phi^{(0)}+\theta^{\mu} \Phi_{\mu}^{(1)}+\frac{1}{2} \theta^{\mu} \theta^{\nu} \Phi_{\mu \nu}^{(2)}$ is

$$
\delta \boldsymbol{\Phi}=\{S, \boldsymbol{\Phi}\}=\delta \Phi^{(0)}-\theta^{\mu} \delta \Phi_{\mu}^{(1)}+\frac{1}{2} \theta^{\mu} \theta^{\nu} \delta \Phi_{\mu \nu}^{(2)}
$$

and the BRST transformation $\delta$ has degree 1. The explicit form of the BRST transformation of each superfield is

$$
\begin{aligned}
& \delta \boldsymbol{\phi}^{i}=\left\{S, \boldsymbol{\phi}^{i}\right\}=\boldsymbol{d} \boldsymbol{\phi}^{i}+f^{i j}(\boldsymbol{\phi}) \boldsymbol{A}_{j}, \\
& \delta \boldsymbol{A}_{i}=\left\{S, \boldsymbol{A}_{i}\right\}=\boldsymbol{d} \boldsymbol{A}_{i}+\frac{1}{2} \frac{\partial f^{j k}}{\partial \boldsymbol{\phi}^{i}}(\boldsymbol{\phi}) \boldsymbol{A}_{j} \boldsymbol{A}_{k} .
\end{aligned}
$$

\footnotetext{
${ }^{f}$ Note that $d \sigma^{\mu}$ is commutative with a Grassmann-odd component field in the nonsuperfield BV formalism, whereas $\theta^{\mu}$ is anticommutative with a Grassmann-odd component field in the superfield formalism.

${ }^{g}$ Precisely, the notation $|\Phi|$ represents the total degree, the sum of the ghost number plus the super form degree of $\Phi$, if it is a graded differential form on a graded manifold. See Appendix.
} 
The (pullback on the) Poisson bracket on a target space is constructed by the double bracket of the super-antibracket:

$$
\{F(\phi), G(\phi)\}_{P B}=\left.f^{i j}(\phi) \frac{\partial F(\phi)}{\partial \phi^{i}} \frac{\partial G(\phi)}{\partial \phi^{j}}\right|_{\phi=\phi}=-\left.\{\{F(\phi), S\}, G(\phi)\}\right|_{\phi=\phi} .
$$

This double bracket is called a derived bracket [95].

This superfield description leads to the AKSZ construction of a topological field theory. In the AKSZ construction, objects in the BV formalism are interpreted as follows: a superfield is a graded manifold; a BV antibracket is a graded symplectic form; and a BV action and the classical master equation are a coboundary operator (homological vector field) $Q$ with $Q^{2}=0$ and its realization by a Hamiltonian function, respectively.

\section{Abelian BF Theories for $i$-Form Gauge Fields in Higher Dimensions}

\subsection{Action}

The superfield constructions discussed in the previous section can be applied to a wide class of TFTs. An abelian BF theory in $n+1$ dimensions is considered as a simple example to show the formulation of the AKSZ construction.

Let us take an $n+1$-dimensional manifold $X_{n+1}$, and let the local coordinates on $X_{n+1}$ be $\sigma^{\mu}$. We consider $i$-form gauge fields with internal index $a_{i}$,

$$
e^{a(i)} \equiv e^{(i) a_{i}}=\frac{1}{i !} d \sigma^{\mu_{1}} \wedge \cdots \wedge d \sigma^{\mu_{i}} e_{\mu_{1} \cdots \mu_{i}}^{a(i)}(\sigma),
$$

for $0 \leq i \leq n$, where we choose the abbreviated notation $e^{a(i)} \cdot a(i)$ denotes an internal index for an $i$-form gauge field. For convenience, we divide the $e^{a(i)}$ 's into two types: $\left(q^{a(i)}, p_{a(n-i)}\right)$, where $q^{a(i)}=e^{a(i)}$ if $0 \leq i \leq\lfloor n / 2\rfloor$; and $p_{a(n-i)}=e^{a(i)}$ if $\lfloor(n+1) / 2\rfloor \leq i \leq n$; where $\lfloor m\rfloor$ is the floor function, which takes the value of the largest integer less than or equal to $m$. If $n$ is even, $q^{a(\lfloor n / 2\rfloor)}$ and $p_{a(n-\lfloor(n+1) / 2\rfloor)}=p_{a(n / 2)}$ are both $n / 2$-form gauge fields. Therefore, we introduce a metric $k_{a(n / 2) b(n / 2)}$ on the internal space of $n / 2$-forms, and we can take $p_{a(n / 2)}=k_{a(n / 2) b(n / 2)} q^{b(n / 2)}$. We denote a 0 -form by $x^{a(0)}\left(=q^{a(0)}=e^{a(0)}\right)$ and an $n$-form by $\xi_{a(0)}\left(=p_{a(0)}=e^{a(n)}\right)$. 
The action $S_{A}$ of an abelian BF theory is the integral of a Lagrangian as $e \wedge d e^{\prime}$. The integral is nonzero only for $(n+1)$-form terms of $e \wedge d e^{\prime}$, since $X_{n+1}$ is in $n+1$ dimensions. Therefore, the action has the following form. If $n=2 m+1$ is odd,

$$
\begin{aligned}
S_{A} & =\int_{X_{n+1}} \sum_{0 \leq i \leq(n-1) / 2, a(i)}(-1)^{n+1-i} p_{a(i)} d q^{a(i)} \\
& =\int_{X_{n+1}}\left((-1)^{n+1} \xi_{a(0)} d x^{a(0)}+\sum_{1 \leq i \leq(n-1) / 2, a(i)}(-1)^{n+1-i} p_{a(i)} d q^{a(i)}\right)
\end{aligned}
$$

and if $n$ is even,

$$
\begin{aligned}
S_{A}= & \int_{X_{n+1}}\left(\sum_{0 \leq i \leq(n-2) / 2, a(i)}(-1)^{n+1-i} p_{a(i)} d q^{a(i)}+(-1)^{\frac{n+1}{2}} k_{a(n / 2) b(n / 2)} q^{a(n / 2)} d q^{b(n / 2)}\right) \\
= & \int_{X_{n+1}}\left((-1)^{n+1} \xi_{a(0)} d x^{a(0)}+\sum_{1 \leq i \leq(n-2) / 2, a(i)}(-1)^{n+1-i} p_{a(i)} d q^{a(i)}\right. \\
& \left.+(-1)^{\frac{n+1}{2}} k_{a(n / 2) b(n / 2)} q^{a(n / 2)} d q^{b(n / 2)}\right) .
\end{aligned}
$$

The sign factors are introduced for later convenience. If we define $p_{a(n / 2)}=k_{a(n / 2) b(n / 2)} q^{a(n / 2)}$, then $S_{A}$ has the same expression for $n$ even or odd:

$$
S_{A}=\sum_{0 \leq i \leq\lfloor n / 2\rfloor, a(i)} \int_{X_{n+1}}(-1)^{n+1-i} p_{a(i)} d q^{a(i)}
$$

This action has the following abelian gauge symmetries:

$$
\delta q^{a(i)}=d q^{(i-1), a(i)}, \quad \delta p_{a(i)}=d p_{a(i)}^{(n-i-1)}
$$

where $q^{(i-1), a(i)}$ is an $(i-1)$-form gauge parameter, and $p_{a(i)}^{(n-i-1)}$ is an $(n-i-1)$-form gauge parameter. These equations are summarized as $\delta e^{a(i)}=d e^{(i-1), a(i)}$, where $e^{(i-1), a(i)}=$ $\left(q^{(i-1), a(i)}, p^{(i-1)} a(n-i)\right)$ is an $(i-1)$-form gauge parameter.

If the $i$-forms are expanded by local fields as $e^{a(i)}(\sigma)=\sum_{k, \mu_{k}} \frac{1}{k !} d \sigma^{\mu_{1}} \wedge \cdots \wedge d \sigma^{\mu_{k}} e^{a(k)}{ }_{\mu_{1} \cdots \mu_{k}}(\sigma)$, the action becomes

$$
S_{A}=\sum_{\substack{0 \leq i \leq\lfloor n / 2\rfloor \\ a(i), \mu_{i}}} \pm \frac{1}{i !(n-i) !} \int_{\mathcal{X}} d^{n+1} \sigma(-1)^{n+1-i} \epsilon^{\mu_{0} \cdots \mu_{n}} p_{a(i) \mu_{i+1} \cdots \mu_{n}} \partial_{\mu_{i}} q^{a(i)}{ }_{\mu_{0} \cdots \mu_{i-1}} .
$$




\subsection{BV Formalism}

In the BV formalism, the ghosts, ghosts for ghosts, and antifields are introduced for each $i$-form gauge field $e^{a(i)}$. First, the gauge parameter $e^{(i-1), a(i)}$ is regarded as the FP ghost of ghost number 1. Moreover, we need the following towers of ghosts for ghosts, because the gauge symmetry is reducible:

$$
\begin{aligned}
& \delta_{0} e^{a(i)}=d e^{(i-1), a(i)}, \\
& \delta_{0} e^{(i-1), a(i)}=d e^{(i-2), a(i)}, \\
& \vdots \\
& \delta_{0} e^{(1), a(i)}=d e^{(0), a(i)}, \\
& \delta_{0} e^{(0), a(i)}=0,
\end{aligned}
$$

where $e^{(k), a(i)}$ is a $k$-form ghost for ghosts, $(k=0, \cdots, i-1)$, of ghost number $i-k$. As usual, these fields are Grassmann-odd (even) if the ghost number is odd (even). We denote the original field by $e^{(i), a(i)}=e^{a(i)}$.

Next, antifields $e^{*(k)} a(i)$ are introduced for all fields and ghosts $e^{(k), a(i)}$ above. An antifield $e^{*(k)}{ }_{a(i)}$ has the same $k$-form as that of the corresponding field $e^{(k), a(i)}$. Note that $\operatorname{gh}(\Phi)+$ $\operatorname{gh}\left(\Phi^{*}\right)=-1$ requires that the antifield has ghost number $k-i-1$. It is convenient to introduce the Hodge dual of an antifield, $e^{+(n+1-k)}{ }_{a(i)}=* e^{(k)}{ }_{a(i)}$, which is an $(n+1-k)$-form of ghost number $k-i-1$. The antibracket is defined as ${ }^{h}$

$$
\begin{aligned}
& \{F, G\} \equiv \sum_{i, k} \int_{X_{n+1}} d^{n+1} \sigma\left(F \frac{\overleftarrow{\partial}}{\partial e^{(k), a(i)}(\sigma)} \frac{\vec{\partial}}{\partial e^{+(n+1-k)} a(i)\left(\sigma^{\prime}\right)} G\right.
\end{aligned}
$$

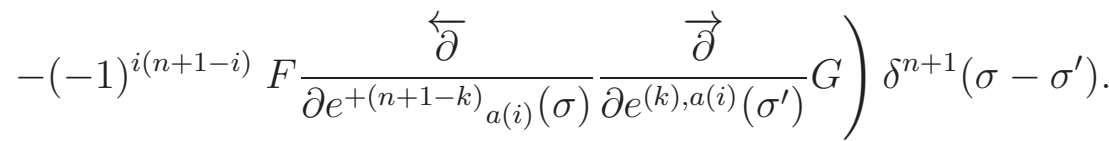

${ }^{h}$ Here, we use simple notation for the functional superderivative, but it will be defined later with more mathematical rigor. 
The BV action is as follows:

$$
\begin{aligned}
S^{(0)} & =S_{A}+\sum_{\Phi}(-1)^{\operatorname{gh} \Phi} \int_{X_{n+1}} d^{n+1} \sigma \Phi^{*} \delta_{0} \Phi \\
& =\sum_{0 \leq i \leq\lfloor n / 2\rfloor, 1 \leq k \leq i} \int_{X_{n+1}}\left((-1)^{n+1-i} p_{a(i)} d q^{a(i)}+(-1)^{i-k} e^{+(n+1-k)}{ }_{a(i)} d e^{(k-1), a(i)}\right) \\
= & \sum_{\substack{0 \leq i \leq\lfloor n / 2\rfloor \\
1 \leq k \leq i}} \int_{X_{n+1}}\left((-1)^{n+1-i} p_{a(i)} d q^{a(i)}+(-1)^{i-k} q^{+(n+1-k)}{ }_{a(i)} d q^{(k-1), a(i)}\right. \\
& \left.+(-1)^{i+k-n} p^{+(k+1), a(i)} d p^{(n-k-1)}{ }_{a(i)}\right) .
\end{aligned}
$$

\subsection{Superfield Formalism}

Let us introduce a supercoordinate $\theta^{\mu}$ of ghost number 1, i.e. of degree 1 . The base $d \sigma^{\mu}$ is replaced by the supercoordinates $\theta^{\mu}$, thus $e^{(k), a(i)}$ and $e^{+(n+1-k)} a(i)$ are replaced by the superfield monomials,

$$
\begin{aligned}
\boldsymbol{e}^{(k), a(i)} & =( \pm) \frac{1}{k !} \theta^{\mu_{1}} \cdots \theta^{\mu_{k}} e_{\mu_{1} \cdots \mu_{k}}^{(k), a(i)}(\sigma) \\
\boldsymbol{e}_{a(i)}^{+(n+1-k)} & =( \pm) \frac{1}{k !} \theta^{\mu_{1}} \cdots \theta^{\mu_{n+1-k}} e_{a(i), \mu_{1} \cdots \mu_{n+1-k}}^{+(n+1-k)}(\sigma)
\end{aligned}
$$

of degree $i$ and of degree $n-i$, respectively. Although sign factors appear in the equations relating the original ghosts and antifields with the superfield components, we do not write them explicitly. Since the relation is one-to-one, we can identify the original fields and ghosts by ghost number and form degree. We define a superfield of degree $i, e^{a(i)}$, where fields and ghosts for an $i$-form gauge field and the antifields for an $(n-i)$-form gauge field are combined [32, 41]. By combining $\boldsymbol{e}^{(k), a(i)}$ and $\boldsymbol{e}^{+(n+1-k)} a(i)$ of degree $i$, we obtain

$$
\begin{aligned}
\boldsymbol{e}^{a(i)} & =\boldsymbol{e}^{(0), a(i)}+\boldsymbol{e}^{(1), a(i)}+\cdots+\boldsymbol{e}^{(i), a(i)}+\boldsymbol{e}^{+(i+1), a(n-i)}+\boldsymbol{e}^{+(i+2), a(n-i)}+\cdots+\boldsymbol{e}^{+(n), a(n-i)} \\
& =\sum_{k=0}^{i} \boldsymbol{e}^{(k), a(i)}+\sum_{k=i+1}^{n} \boldsymbol{e}^{+(k), a(n-i)}
\end{aligned}
$$

where $0 \leq i \leq n$. Note that the internal indices $a(i)$ and $a(n-i)$ are equivalent, since we are considering a BF theory.

Let us denote the super-antibracket conjugate pair by $\left(\boldsymbol{e}^{a(i)}, \boldsymbol{e}^{a(n-i)}\right)=\left(\boldsymbol{q}^{a(i)}, \boldsymbol{p}_{a(i)}\right)$. Then, 
the superfields can be written as follows:

$$
\begin{aligned}
& \boldsymbol{q}^{a(i)}=\sum_{k=0}^{i} \boldsymbol{q}^{(k), a(i)}+\sum_{k=i+1}^{n} \boldsymbol{p}^{(k), a(n-i)}, \\
& \boldsymbol{p}_{a(i)}=\sum_{k=0}^{i} \boldsymbol{p}_{a(i)}^{(k)}+\sum_{k=i+1}^{n} \boldsymbol{q}_{a(n-i)}^{(k)} .
\end{aligned}
$$

If $n$ is even, the $n / 2$-form part has a special relation, $\boldsymbol{p}_{a(n / 2)}=k_{a(n / 2) b(n / 2)} \boldsymbol{q}^{b(n / 2)}$. Therefore, $\boldsymbol{q}^{a(n / 2)}$ contains both ghosts and antifields for an $(n / 2)$-form gauge field $\boldsymbol{q}^{(n / 2), a(n / 2)}$ :

$$
\boldsymbol{q}^{a(n / 2)}=\sum_{k=0}^{n / 2} \boldsymbol{q}^{(k), a(n / 2)}+\sum_{k=n / 2+1}^{n} k^{a(n / 2) b(n / 2)} \boldsymbol{q}_{(k), b(n / 2)}
$$

If we use superfields, the antibrackets and the BV action are simplified. The antibracket (3.25) can be rewritten using superfields (3.29) as follows:

$$
\begin{aligned}
\{F, G\} \equiv & \int_{X_{n+1}} d^{n+1} \sigma d^{n+1} \theta\left(F \frac{\overleftarrow{\partial}}{\partial \boldsymbol{q}^{a(i)}(\sigma, \theta)} \frac{\vec{\partial}}{\partial \boldsymbol{p}_{a(i)}\left(\sigma^{\prime}, \theta^{\prime}\right)} G\right. \\
& \left.\quad-(-1)^{i(n-i)} F \frac{\overleftarrow{\partial}}{\partial \boldsymbol{p}_{a(i)}(\sigma, \theta)} \frac{\vec{\partial}}{\partial \boldsymbol{q}^{a(i)}\left(\sigma^{\prime}, \theta^{\prime}\right)} G\right) \delta^{n+1}\left(\sigma-\sigma^{\prime}\right) \delta^{n+1}\left(\theta-\theta^{\prime}\right) \\
= & \int_{X_{n+1}} d^{n+1} \sigma d^{n+1} \theta\left(F \frac{\overleftarrow{\partial}}{\partial \boldsymbol{e}^{a(i)}(\sigma)} \boldsymbol{\omega}^{a(i) b(j)} \frac{\vec{\partial}}{\partial \boldsymbol{e}^{b(j)}\left(\sigma^{\prime}\right)} G\right) \delta^{n+1}\left(\sigma-\sigma^{\prime}\right) \delta^{n+1}\left(\theta-\theta^{\prime}\right)
\end{aligned}
$$

Note that $\boldsymbol{\omega}^{a(i) b(j)}$ is the inverse of the graded symplectic structure on superfields. The complicated BV action (3.26) can be simplified as the BV superaction as follows:

$$
\begin{aligned}
S^{(0)} & =\sum_{0 \leq i \leq\lfloor n / 2\rfloor} \int d^{n+1} \sigma d^{n+1} \theta(-1)^{n+1-i} \boldsymbol{p}_{a(i)} \boldsymbol{d \boldsymbol { q } ^ { a ( i ) }} \\
& =\sum_{0 \leq i \leq n} \int \mu \frac{1}{2} \boldsymbol{e}^{a(i)} \boldsymbol{\omega}_{a(i) b(j)} d \boldsymbol{e}^{b(j)}
\end{aligned}
$$

where $\mu$ is the Berezin measure on the supermanifold.

As in the previous section, we apply deformation theory to the BV action $S^{(0)}$ and obtain all possible consistent terms of the BV action $S_{I}$ in BF theory. Deformation theory in the superfield formalism yields the same result as in the nonsuperfield BV formalism, in the case of a topological field theory. [70, 71] Therefore, below we will compute only in the superfield formalism. 
The topological field theories constructed in Sections 2 and 3 have the same structures: superfields, antibrackets and BV actions. These are formulated in a unified way by $Q P$ manifolds and the structure becomes more transparent.

\section{QP-manifolds}

\subsection{Definition}

A QP-manifold, which is also called a differential graded symplectic manifold, is a key structure for the AKSZ construction of a topological field theory. This section and the next are devoted to providing the fundamentals of the formulation. For further reading, we refer to Refs. [35, 124, 121, 42].

A graded manifold is the mathematical counterpart to a superfield, which is defined as a ringed space with a structure sheaf of a graded commutative algebra over an ordinary smooth manifold $M$. It is defined locally using even and odd coordinates. This grading is compatible with supermanifold grading, that is, a variable of even degree is commutative, and one of odd degree is anticommutative. The grading is called the degree. $\mathcal{M}$ is locally isomorphic to $C^{\infty}(U) \otimes S^{\cdot}(V)$, where $U$ is a local chart on $M, V$ is a graded vector space, and $S^{\cdot}(V)$ is a free graded commutative algebra on $V$. We refer to Refs. [27, 111, 144] for a rigorous definition and a discussion of the properties of a supermanifold. The formulas for the graded differential calculus are summarized in Appendix A.

The grading is assumed to be nonnegative in this lecture ${ }^{i}$ and a graded manifold with a nonnegative grading is called an $N$-manifold.

The mathematical structure corresponding to the antibracket is a P-structure. Thus, an $\mathrm{N}$-manifold equipped with a graded symplectic structure $\omega$ of degree $n$ is called a P-manifold of degree $n,(\mathcal{M}, \omega)$, and $\omega$ is a P-structure. The graded Poisson bracket on $C^{\infty}(\mathcal{M})$ is defined from the graded symplectic structure $\omega$ on $\mathcal{M}$ as

$$
\{f, g\}=(-1)^{|f|+n} \iota_{X_{f}} \delta g=(-1)^{|f|+n+1} \iota_{X_{f}} \iota_{X_{g}} \omega
$$

\footnotetext{
$\bar{i}$ Though we do not consider a grading with negative degree in this article, there exist sigma models on target graded manifolds with negative degree. [77, 156]
} 
for $f, g \in C^{\infty}(\mathcal{M})$, where the Hamiltonian vector field $X_{f}$ is defined by the equation $\iota_{X_{f}} \omega=$ $-\delta f$.

Finally, a $Q$-structure corresponding to a BV action is introduced. Let $(\mathcal{M}, \omega)$ be a $P$ manifold of degree $n$. We require that there is a differential $Q$ of degree +1 with $Q^{2}=0$ on $\mathcal{M}$. This $Q$ is called a Q-structure.

Definition 4.1 The triple $(\mathcal{M}, \omega, Q)$ is called a QP-manifold of degree $n$, and its structure is called a QP-structure, if $\omega$ and $Q$ are compatible, that is, $L_{Q} \omega=0$. [132, 133]

$Q$ is also called a homological vector field. In fact, $Q$ is a Grassmann-odd vector field on $\mathcal{M}$. We take a generator $\Theta \in C^{\infty}(\mathcal{M})$ of $Q$ with respect to the graded Poisson bracket, $\{-,-\}$, satisfying

$$
Q=\{\Theta,-\}
$$

$\Theta$ has degree $n+1$ and is called homological function, or Q-structure function. $\Theta$ is also called Hamiltonian $j$ The differential condition, $Q^{2}=0$, implies that $\Theta$ is a solution of the classical master equation,

$$
\{\Theta, \Theta\}=0
$$

\subsection{Notation}

We will now introduce the notation for graded manifolds. Let $V$ be an ordinary vector space. Then $V[n]$ is a vector space in which the degree is shifted by $n$. More generally, if $V_{m}$ is a graded vector space of degree $m$, the elements of $V_{m}[n]$ are of degree $m+n$ (this is also denoted by $\left.V_{m+n}=V_{m}[n]\right)$. If $V$ has degree $n$, the dual space $V^{*}$ has degree $-n$. The product of $u \in V_{m}$ and $v \in V_{n}$ is graded commutative, $u v=(-1)^{m n} v u$.

Let $M$ be an ordinary smooth manifold. Given a vector bundle $E \longrightarrow M, E[n]$ is a graded manifold assigning degree $n$ to the fiber variables, i.e., a base variable has degree 0 , and a fiber variable has degree $n$. If the degree of the fiber is shifted by $n$, graded tangent and cotangent bundles are denoted by $T[n] M$ and $T^{*}[n] M$, respectively.

This notation is generalized to the case that both a smooth manifold $M$ and its fiber are graded. $E[n]$ means that the degree of the fiber is shifted by $n$. Note that $T M[1]$ is a

${ }^{j}$ In fact, if the degree of a QP-manifold is positive, there always exists a generator $\Theta$ for the Q-structure differential $Q$ [124]. 
tangent bundle for which the base and fiber degrees are 1 and 1 , which is denoted by $(1,1)$. Considering the duality of $V$ and $V^{*}$, we then have that $T^{*} M[1]$ is a cotangent bundle for which the base and fiber degrees are $(1,-1)$. Therefore, $T^{*}[n] M[1]$ is a cotangent bundle of degrees $(1, n-1)$.

Let us consider a typical example: a double vector bundle $T^{*} E$, which is the cotangent bundle of a vector bundle. We take local coordinates on $E,\left(x^{i}, q^{a}\right)$, where $x^{i}$ is a coordinate on $M$, and $q^{a}$ is a coordinate on the fiber. We also take dual coordinates $\left(\xi_{i}, p_{a}\right)$ on the cotangent space. If we consider the graded bundle $T^{*}[n] E[1]$, the coordinates $\left(x^{i}, q^{a}\right)$ have degrees $(0,1)$ and $\left(\xi_{i}, p_{a}\right)$ have degrees $(0+n,-1+n)=(n, n-1) . \mid k$

We can see that $C^{\infty}(E[1])$, the space of functions on $E[1]$, is equivalent to the space of sections of the exterior algebra, $\wedge^{\bullet} E, C^{\infty}(E[1])=\Gamma\left(\wedge^{\bullet} E\right)$, if we identify the local coordinates of degree 1 with the basis of the exterior algebra. Let $e^{a}$ be a local basis of the sections of $E$. Then, a function

$$
\frac{1}{s !} f_{a_{1} \cdots a_{s}}(x) q^{a_{1}} \cdots q^{a_{s}} \in C^{\infty}(E[1])
$$

can be identified with

$$
\frac{1}{s !} f_{a_{1} \cdots a_{s}}(x) e^{a_{1}} \wedge \cdots \wedge e^{a_{s}} \in \Gamma\left(\wedge^{\bullet} E\right)
$$

\section{Examples of QP-Manifolds}

Typical examples of QP-manifolds are listed below.

\subsection{Lie Algebra and Lie Algebroid as QP-manifold of degree $n$}

\subsubsection{Lie Algebra}

Let $n \geq 1$. For an arbitrary $n$, a Lie algebra becomes a QP-manifold of degree $n$ on a point $M=\{p t\}$.

\footnotetext{
${ }^{k}$ For notation $[n]$, we consider degree by $\boldsymbol{Z}$-grading. On the other hand, we can regard a graded manifold as a supermanifold by considering the degree modulo 2 . In this case, the shifting of odd and even degrees is denoted by П. For example, ПTM is a tangent bundle in which the degree of the fiber is odd. There is a natural isomorphism, $\Pi T M \simeq T[1] M$.
} 
Let $\mathfrak{g}$ be a Lie algebra with a Lie bracket $[-,-]$. Then, $T^{*}[n] \mathfrak{g}[1] \simeq \mathfrak{g}[1] \oplus \mathfrak{g}^{*}[n-1]$ is a P-manifold of degree $n$ with graded symplectic structure induced by a canonical symplectic structure on $T^{*} \mathfrak{g}$. We take local coordinates as follows: $q^{a} \in \mathfrak{g}[1]$ of degree 1 , and $p_{a} \in \mathfrak{g}^{*}[n-1]$ of degree $n-1$. A P-structure $\omega=(-1)^{n|q|} \delta q^{a} \wedge \delta p_{a}$ is of degree $n$, and it is induced by the canonical symplectic structure on $T^{*} \mathfrak{g} \simeq \mathfrak{g} \oplus \mathfrak{g}^{*}$ by shifting the degree of the coordinates. Taking a Cartan form $\Theta=\frac{1}{2}\langle p,[q, q]\rangle=\frac{1}{2} f^{a}{ }_{b c} p_{a} q^{b} q^{c}$, where $\langle-,-\rangle$ is the canonical pairing of $\mathfrak{g}$ and $\mathfrak{g}^{*}, f_{b c}^{a}$ is the structure constant, then, $\Theta$ defines a Q-structure, since it satisfies $\{\Theta, \Theta\}=0$ due to the Lie algebra structure.

\subsubsection{Lie Algebroid}

A Lie algebroid has been defined in Definition 2.2. A Lie algebroid has a realization by a QP-manifold of degree $n$ for every $n$.

Let $n \geq 2$. Let $E$ be a vector bundle over $M$, and let $\mathcal{M}=T^{*}[n] E[1]$ be a graded manifold of degree $n$. We take local coordinates $\left(x^{i}, q^{a}, p_{a}, \xi_{i}\right)$ of degrees $(0,1, n-1, n)$. The P-structure $\omega$ is a graded differential form of degree $n$ and is locally written as

$$
\omega=\delta x^{i} \wedge \delta \xi_{i}+(-1)^{n|q|} \delta q^{a} \wedge \delta p_{a}
$$

The Q-structure function is of degree $n+1$, and we have

$$
\Theta=f_{1}^{i}{ }_{a}(x) \xi_{i} q^{a}+\frac{1}{2} f_{2}{ }^{a}{ }_{b c}(x) p_{a} q^{b} q^{c},
$$

where the $f_{i}$ 's are functions of $x$. The Q-structure condition $\{\Theta, \Theta\}=0$ imposes the following relations:

$$
\begin{aligned}
& f_{1}{ }^{k}{ }_{b} \frac{\partial f_{1}^{i} a}{\partial x^{k}}-f_{1}{ }^{k}{ }_{a} \frac{\partial f_{1}{ }^{i} b}{\partial x^{k}}+f_{1}{ }^{i}{ }_{c} f_{2}{ }^{c}{ }_{a b}=0, \\
& f_{1}{ }^{k}\left[d \frac{\left.\partial f_{2}{ }^{a} b c\right]}{\partial x^{k}}-f_{2}{ }^{a}{ }_{e[b} f_{2}{ }^{e} c d\right]=0 .
\end{aligned}
$$

(5.39) and (5.40) are the same conditions as for a Lie algebroid, (2.14) and (2.15), where $f_{1}{ }^{i}{ }_{a}=\rho^{i}{ }_{a}$ and $f_{2}{ }^{a} b c=-f^{a}{ }_{b c}$.

For $n=1$, we need a slightly different realization, which appeared in Ref. [20].

\section{$5.2 n=1$}

In general, a QP-manifold of $n=1$ defines a Poisson structure. We can also realize a complex structure using $n=1$. Here, we give their constructions. 


\subsubsection{Poisson Structure}

A P-manifold $\mathcal{M}$ of $n=1$ has the two degrees $(0,1)$, and it is canonically isomorphic to the cotangent bundle $\mathcal{M}=T^{*}[1] M$, over the smooth manifold $M$.

On $T^{*}[1] M$, we take local coordinates $\left(x^{i}, \xi_{i}\right)$ of degrees $(0,1)$; here, $x^{i}$ is a coordinate of the base manifold $M$, and $\xi_{i}$ is a coordinate of the fiber. Note that $\xi_{i}$ is an odd element: $\xi_{i} \xi_{j}=-\xi_{j} \xi_{i}$. The P-structure is $\omega=\delta x^{i} \wedge \delta \xi_{i}$. For $n=1$, the graded Poisson bracket $\{-,-\}$ is isomorphic to the Schouten-Nijenhuis bracket. Since the Q-structure function $\Theta$ has degree two, the general form is $\Theta=\frac{1}{2} f^{i j}(x) \xi_{i} \xi_{j}$, where $f^{i j}(x)$ is an arbitrary function of $x$. The classical master equation, $\{\Theta, \Theta\}=0$, imposes the following condition on $f^{i j}(x)$ :

$$
\frac{\partial f^{i j}(x)}{\partial x^{l}} f^{l k}(x)+(i j k \text { cyclic })=0 .
$$

The Q-structure $\Theta$ with Equation (5.41) is called a Poisson bivector field.

If $f^{i j}$ satisfies equation (5.41), then the derived bracket defines a Poisson bracket on $M$ :

$$
\{F, G\}_{P B}=f^{i j}(x) \frac{\partial F}{\partial x^{i}} \frac{\partial G}{\partial x^{j}}=-\{\{F, \Theta\}, G\} .
$$

Equation (5.41) corresponds to the Jacobi identity of this Poisson bracket.

Conversely, assume a Poisson bracket $\{F, G\}_{P B}$ on $M$. The Poisson bracket can be locally written as $f^{i j}(x) \frac{\partial F}{\partial x^{i}} \frac{\partial G}{\partial x^{j}}$. Then, $\Theta=\frac{1}{2} f^{i j}(x) \xi_{i} \xi_{j}$ satisfies the classical master equation and is a Q-structure.

Thus, a QP-manifold of degree $1, T^{*}[1] M$, defines a Poisson structure on $M$. This QPmanifold of degree 1 is also regarded as a Lie algebroid on $T^{*} M$, according to Definition 2.2 .

\subsubsection{Complex Structure}

Let $M$ be a complex manifold of real dimension $d$. A linear transformation $J: T M \longrightarrow T M$ is called a complex structure if the following two conditions are satisfied:

1) $J^{2}=-1$

2) For $X, Y \in T M, p r_{\mp}\left[p r_{ \pm} X, p r_{ \pm} Y\right]=0, \quad$ (integrability condition) where $p r_{ \pm}$is the projection onto the $\pm \sqrt{-1}$ eigenbundles in $T M$, and $[-,-]$ is the Lie bracket of vector fields. We take a local coordinate expression of $J, J^{i}{ }_{j}(x)$, which is a rank $(1,1)$ tensor. 
In order to formulate a complex structure as a QP-manifold we take the graded manifold $\mathcal{M}=T^{*}[1] T[1] M$. This double vector bundle is locally isomorphic to $U \times \boldsymbol{R}^{d}[1] \times \boldsymbol{R}^{d}[1] \times \boldsymbol{R}^{d}[0]$, where $U$ is a local chart on $M$. Let us take local coordinates on the local chart as $\left(x^{i}, \xi_{i}, q^{i}, p_{i}\right)$ of degree $(0,1,1,0)$. The P-structure is defined as

$$
\omega=\delta x^{i} \wedge \delta \xi_{i}+\delta p_{i} \wedge \delta q^{i}
$$

If we take the Q-structure as

$$
\begin{aligned}
\Theta & =J^{i}{ }_{j}(x) \xi_{i} q^{j}+\frac{\partial{J^{i}}_{k}}{\partial x^{j}}(x) p_{i} q^{j} q^{k} \\
& =\left(\xi_{i} q^{i}\right)\left(\begin{array}{cc}
0 & \frac{1}{2} J^{i}{ }_{j}(x) \\
-\frac{1}{2} J^{j}{ }_{i}(x) & \frac{\partial J^{i} k}{\partial x^{j}}(x) p_{i}
\end{array}\right)\left(\begin{array}{l}
\xi_{j} \\
q^{j}
\end{array}\right),
\end{aligned}
$$

then $\{\Theta, \Theta\}=0$ is equivalent to condition 2) in the definition of the complex structure $J$.

\section{$5.3 n=2$}

The following theorem is well known. [122, 123]

Theorem 5.1 A QP-structure of degree 2 is equivalent to the Courant algebroid on a vector bundle $E$ over a smooth manifold $M$.

We explain this in detail.

\subsubsection{Courant Algebroid}

For $n=2$, the P-structure $\omega$ is an even form of degree 2. The Q-structure function $\Theta$ has degree 3. $Q^{2}=0$ defines a Courant algebroid [44, 105] structure on a vector bundle $E$.

First, let us introduce the most general form of the QP-manifold of degree $2,(\mathcal{M}, \omega, \Theta)$. We denote the local coordinates of $\mathcal{M}$ as $\left(x^{i}, \eta^{a}, \xi_{i}\right)$ of degrees $(0,1,2)$. The P-structure $\omega$ of degree 2 can be locally written as

$$
\omega=\delta x^{i} \wedge \delta \xi_{i}+\frac{k_{a b}}{2} \delta \eta^{a} \wedge \delta \eta^{b},
$$

where we have introduced a metric $k_{a b}$ on the degree one subspace. The general form of the Q-structure function of degree 3 is

$$
\Theta=f_{1{ }^{i} a}(x) \xi_{i} \eta^{a}+\frac{1}{3 !} f_{2 a b c}(x) \eta^{a} \eta^{b} \eta^{c},
$$


where $f_{1}{ }^{i}{ }_{a}(x)$ and $f_{2 a b c}(x)$ are local functions of $x$. The Q-structure condition $\{\Theta, \Theta\}=0$ imposes the following relations on these functions:

$$
\begin{aligned}
& k^{a b} f_{1}{ }^{i}{ }_{a} f_{1}{ }^{j} b=0 \\
& \frac{\partial f_{1}{ }^{i} b}{\partial x^{j}} f_{1}{ }^{j}{ }_{c}-\frac{\partial f_{1}{ }^{i} c}{\partial x^{j}} f_{1}{ }^{j}{ }_{b}+k^{e f} f_{1}{ }^{i}{ }_{e} f_{2 f b c}=0 \\
& \left(f_{1}{ }^{i}{ }_{d} \frac{\partial f_{2 a b c}}{\partial x^{i}}-f_{1}{ }^{i}{ }_{c} \frac{\partial f_{2 d a b}}{\partial x^{i}}+f_{1}{ }^{i} b \frac{\partial f_{2 c d a}}{\partial x^{i}}-f_{1}{ }^{i}{ }_{a} \frac{\partial f_{2 b c d}}{\partial x^{i}}\right) \\
& \quad+k^{e f}\left(f_{2 e a b} f_{2 c d f}+f_{2 e a c} f_{2 d b f}+f_{2 e a d} f_{2 b c f}\right)=0 .
\end{aligned}
$$

We can prove that these identities (5.45) are the same as the local coordinate expressions of the Courant algebroid conditions on a vector bundle $E$. The Courant algebroid is defined as:

Definition 5.2 A Courant algebroid is a vector bundle $E \longrightarrow M$, and it has a nondegenerate symmetric bilinear form $\langle\cdot, \cdot\rangle$ on the bundle, a bilinear operation $\circ$ on $\Gamma(E)$, and a bundle map called an anchor map, $\rho: E \longrightarrow T M$, satisfying the following properties:

$$
\begin{array}{ll}
1, & e_{1} \circ\left(e_{2} \circ e_{3}\right)=\left(e_{1} \circ e_{2}\right) \circ e_{3}+e_{2} \circ\left(e_{1} \circ e_{3}\right), \\
2, & \rho\left(e_{1} \circ e_{2}\right)=\left[\rho\left(e_{1}\right), \rho\left(e_{2}\right)\right], \\
3, & e_{1} \circ F e_{2}=F\left(e_{1} \circ e_{2}\right)+\left(\rho\left(e_{1}\right) F\right) e_{2}, \\
4, & e_{1} \circ e_{2}=\frac{1}{2} \mathcal{D}\left\langle e_{1}, e_{2}\right\rangle, \\
5, & \rho\left(e_{1}\right)\left\langle e_{2}, e_{3}\right\rangle=\left\langle e_{1} \circ e_{2}, e_{3}\right\rangle+\left\langle e_{2}, e_{1} \circ e_{3}\right\rangle,
\end{array}
$$

where $e_{1}, e_{2}$, and $e_{3}$ are sections of $E, F$ is a function on $M$ and $\mathcal{D}$ is a map from the space of functions on $M$ to $\Gamma(E)$, defined as $\langle\mathcal{D} F, e\rangle=\rho(e) F$.

Let $x^{i}$ be a local coordinate on $M$, and let $e^{a}$ be a local coordinate on the fiber of $E$. We can write each operation on the local basis $x^{i}, e^{a}$, as follows:

$$
\begin{aligned}
& e^{a} \circ e^{b}=k^{a d} k^{b e} f_{2 d e c}(x) e^{c}, \\
& \left\langle e^{a}, e^{b}\right\rangle=k^{a b}, \\
& \rho\left(e^{a}\right) F(x)=-k^{a b} f_{1 b}^{i}(x) \frac{\partial F}{\partial x^{i}}(x),
\end{aligned}
$$

where $f_{1}$ and $f_{2}$ are local functions of $x$. Substituting these expressions into the relations given in equations (5.46)-(5.50), we obtain the identities (5.45). 
These operations can be constructed directly from a QP-manifold $\mathcal{M}$, without introducing local coordinates. For this, we identify a section $e$ with an odd element $\eta$ in supergeometry computations using the shift functor [1]. Then, the operations of the Courant algebroid can be represented as

$$
\begin{aligned}
& e_{1} \circ e_{2} \equiv-\left\{\left\{e_{1}, \Theta\right\}, e_{2}\right\}, \\
& \left\langle e_{1}, e_{2}\right\rangle \equiv\left\{e_{1}, e_{2}\right\}, \\
& \rho(e) F \equiv\{e,\{\Theta, F\}\}, \\
& \mathcal{D}(*) \equiv\{\Theta, *\},
\end{aligned}
$$

where $F(x)$ is a function of degree 0 and $e=\eta$ is a function of degree 1 . We can also prove that $\{\Theta, \Theta\}=0$ gives the Courant algebroid structure (5.46)-(5.50) without using local coordinates. Finally, a vector bundle $E$ is constructed from a graded manifold $\mathcal{M}$ by a natural filtration of degree $\mathcal{M} \longrightarrow E[1] \longrightarrow M$.

An important example of a Courant algebroid is the direct sum of the tangent and cotangent bundles, $E=T M \oplus T^{*} M$. The bilinear operation is defined as

$$
(X+\alpha) \circ(Y+\beta)=[X+\alpha, Y+\beta]_{D}=[X, Y]+L_{X} \beta-\iota_{Y} d \alpha .
$$

Here, $X, Y \in T M$ are vector fields, $\alpha, \beta \in T^{*} M$ are 1-forms, $[-,-]$ is the ordinary Lie bracket on a vector field, $L_{X}$ is the Lie derivative, and $\iota_{X}$ is the interior product, respectively. The bracket (5.52) is called the Dorfman bracket, and generally it is not antisymmetric. The Dorfman bracket is the most general bilinear form on $T M \oplus T^{*} M$ without background flux, which satisfies the Leibniz identity ${ }^{l}$ The antisymmetrization of the Dorfman bracket is called the Courant bracket. The Courant bracket is antisymmetric, but it does not satisfy the Jacobi identity. The symmetric form is $\langle X+\alpha, Y+\beta\rangle=\iota_{X} \beta+\iota_{Y} \alpha$ and the anchor map $\rho$ is the natural projection to $T M$ :

$$
\rho(X+\alpha)=X
$$

The corresponding QP-manifold is $\mathcal{M}=T^{*}[2] T^{*}[1] M$. The local Darboux coordinates are $\left(x^{i}, q^{i}, p_{i}, \xi_{i}\right)$, which have degrees $(0,1,1,2) \cdot{ }^{m}$ Here, $q^{i}$ is a fiber coordinate of $T[1] M, p_{i}$ a fiber

\footnotetext{
$l$ Note that $\circ$ is not necessarily assumed to be antisymmetric. For a nonantisymmetric bracket, equation
} (5.46) is called Leibniz identity instead of Jacobi identity.

${ }^{m}$ We can compare this formulation with the most general form of a QP-manifold by taking $\eta^{a}=\left(q^{i}, p_{i}\right)$. 
coordinate of $T^{*}[1] M$, and $\xi_{i}$ a fiber coordinate of $T^{*}[2] M$, respectively. With degree shifting, $T M \oplus T^{*} M$ is naturally embedded into $T^{*}[2] T^{*}[1] M$ as $\left(x^{i}, d x^{i}, \frac{\partial}{\partial x^{i}}, 0\right) \mapsto\left(x^{i}, q^{i}, p_{i}, \xi_{i}\right)$. The Courant algebroid structure on $T M \oplus T^{*} M$ is constructed from equation (5.51). The Dorfman bracket can be found via a derived bracket as $[-,-]_{D}=\{\{-, \Theta\},-\}$ with $\Theta=\xi_{i} q^{i}$. It means that $f_{1}{ }^{i}{ }_{j}=\delta^{i}{ }_{j}$ and $f_{2 i j k}=0$. This Courant algebroid is also called the standard Courant algebroid.

There is a freedom to introduce a closed 3-form $H(x)$ as an extra datum. If the Dorfman bracket is modified by $H(x)$ as $(X+\alpha) \circ(Y+\beta)=[X+\alpha, Y+\beta]_{D}=[X, Y]+L_{X} \beta-i_{Y} d \alpha+$ $i_{X} i_{Y} H$, the Courant algebroid structure is preserved. This is called the Dorfman bracket with a 3-form $H$. The P-structure remains the same, but $\Theta$ is modified as $\Theta=\xi_{i} q^{i}+\frac{1}{3 !} H_{i j k}(x) q^{i} q^{j} q^{k}$, where $H(x)=\frac{1}{3 !} H_{i j k}(x) d x^{i} \wedge d x^{j} \wedge d x^{k} .\{\Theta, \Theta\}=0$ is equivalent to $d H=0$. This is called the standard Courant algebroid with H-flux.

There is an equivalent definition of the Courant algebroid [96], and it is closer to the construction from a QP-manifold.

Definition 5.3 Let $E$ be a vector bundle over $M$ that is equipped with a pseudo-Euclidean metric $(-,-)$, a bundle map $\rho: E \longrightarrow T M$, and a binary bracket $[-,-]_{D}$ on $\Gamma(E)$. The bundle is called the Courant algebroid if the following three conditions are satisfied:

$$
\begin{aligned}
{\left[e_{1},\left[e_{2}, e_{3}\right]_{D}\right]_{D} } & =\left[\left[e_{1}, e_{2}\right]_{D}, e_{3}\right]_{D}+\left[e_{2},\left[e_{1}, e_{3}\right]_{D}\right]_{D} \\
\rho\left(e_{1}\right)\left(e_{2}, e_{3}\right) & =\left(\left[e_{1}, e_{2}\right]_{D}, e_{3}\right)+\left(e_{2},\left[e_{1}, e_{3}\right]_{D}\right) \\
\rho\left(e_{1}\right)\left(e_{2}, e_{3}\right) & =\left(e_{1},\left[e_{2}, e_{3}\right]_{D}+\left[e_{3}, e_{2}\right]_{D}\right)
\end{aligned}
$$

where $e_{1}, e_{2}, e_{3} \in \Gamma(E)$.

We can prove that Definitions 5.2 and 5.3 are equivalent if the operations are identified as $e_{1} \circ e_{2}=\left[e_{1}, e_{2}\right]_{D},\left\langle e_{1}, e_{2}\right\rangle=\left(e_{1}, e_{2}\right)$, with the same bundle map $\rho$.

Dirac structure A Dirac structure can be formulated in QP-manifold language. A Dirac structure is a Lie algebroid, which is a substructure of a Courant algebroid, defined by:

Definition 5.4 A Dirac structure $\mathcal{L}$ is a maximally isotropic subbundle of a Courant algebroid E, whose sections are closed under the Dorfman bracket. That is,

$$
\begin{aligned}
& \left\langle e_{1}, e_{2}\right\rangle=0 \quad(\text { isotropic }) \\
& {\left[e_{1}, e_{2}\right]_{C} \in \Gamma(\mathcal{L}) \quad(\text { closed })}
\end{aligned}
$$


for $e_{1}, e_{2} \in \Gamma(\mathcal{L})$, where $\left[e_{1}, e_{2}\right]_{C}=\left[e_{1}, e_{2}\right]_{D}-\left[e_{2}, e_{1}\right]_{D}$ is the Courant bracket.

In QP-manifold language, the sections $\Gamma\left(\wedge^{\bullet} E\right)$ are identified with functions on the QPmanifold $C^{\infty}(\mathcal{M})$. Then, the sections of the Dirac structure $\Gamma(\mathcal{L})$ are the functions with the conditions corresponding to (5.57) and (5.58), which are commutativity under the Pstructure $\{-,-\}$, and closedness under the derived bracket $\{\{-, \Theta\},-\}$, respectively.

The Dirac structure on the complexification of the Courant algebroid, $\left(T M \oplus T^{*} M\right) \otimes \boldsymbol{C}$, defines a generalized complex structure. [64, 57]

\section{$5.4 \quad n \geq 3$}

We now define the algebraic and geometric structures which appear for $n \geq 3$ and give some examples. An earlier analysis of the unification of algebraic and geometric structures induced by higher QP-structures has been found in Ref. [136].

Definition 5.5 A vector bundle $\left(E, \rho,[-,-]_{L}\right)$ is called an algebroid if there is a bilinear operation $[-,-]_{L}: \Gamma(E) \times \Gamma(E) \rightarrow \Gamma(E)$, and a bundle map $\rho: E \rightarrow T M$ satisfying the following conditions:

$$
\begin{aligned}
& \rho\left[e_{1}, e_{2}\right]_{L}=\left[\rho\left(e_{1}\right), \rho\left(e_{2}\right)\right], \\
& {\left[e_{1}, F e_{2}\right]_{L}=F\left[e_{1}, e_{2}\right]_{L}+\rho\left(e_{1}\right)(F) e_{2},}
\end{aligned}
$$

where $F \in C^{\infty}(M)$ and $\left[\rho\left(e_{1}\right), \rho\left(e_{2}\right)\right]$ is the usual Lie bracket on $\Gamma(T M)$. Note that $[-,-]_{L}$ need not be antisymmetric, and it need not satisfy the Jacobi identity. $\rho$ is called anchor map.

Definition 5.6 An algebroid $\left(E, \rho,[-,-]_{L}\right)$ is called a Leibniz algebroid (or a Loday algebroid) if there is a bracket product $\left[e_{1}, e_{2}\right]_{L}$ satisfying the Leibniz identity:

$$
\left[e_{1},\left[e_{2}, e_{3}\right]_{L}\right]_{L}=\left[\left[e_{1}, e_{2}\right]_{L}, e_{3}\right]_{L}+\left[e_{2},\left[e_{1}, e_{3}\right]_{L}\right]_{L},
$$

where $e_{1}, e_{2}, e_{3} \in \Gamma(E)$.

If the base manifold is a point $M=\{p t\}$ and $\rho=0$, then the Leibniz algebroid reduces to a linear algebra, which is called Leibniz (Loday) algebra [107, 106]. A Leibniz algebra is a Lie algebra if the Leibniz bracket $[-,-]_{L}$ is antisymmetric. Lie algebroids and Courant algebroids are also Leibniz algebroids. The Lie bracket $[-,-]$ of the Lie algebroid and the 
Dorfman bracket $[-,-]_{D}$ of the Courant algebroid are identified as special cases of the Leibniz bracket $[-,-]_{L}$. Equation (5.54) of the Dorfman bracket is equivalent to equation (5.61).

The correspondence of a Leibniz algebroid to a homological vector field on a graded manifold is discussed in Ref. [53]. The following theorem has been presented in Ref. [100].

Theorem 5.7 Let $n>1$. Functions of degree $n-1$ on a QP-manifold can be identified with sections of a vector bundle. The QP-structure induces a Leibniz algebroid structure on a vector bundle $E$.

Let $x$ be an element of degree 0 , and let $e^{(n-1)}$ be an element of degree $n-1$. If we define

$$
\begin{aligned}
{\left[e_{1}, e_{2}\right]_{L} } & =-\left\{\left\{e_{1}^{(n-1)}, \Theta\right\}, e_{2}^{(n-1)}\right\}, \\
\rho(e) F(x) & =(-1)^{n}\left\{\left\{e^{(n-1)}, \Theta\right\}, F(x)\right\},
\end{aligned}
$$

then $e^{(n-1)}$ is identified with a section of a vector bundle, and $[-,-]_{L}$ and $\rho$ satisfy the conditions in the definition of a Leibniz algebroid given by equations (5.59), (5.60) and (5.61).

\subsection{1 $n=3$}

Let $n=3$. Let $(\mathcal{M}, \omega, \Theta)$ be a QP-manifold of degree 3. $\mathcal{M}$ has a natural filtration of degree $\mathcal{M} \longrightarrow \mathcal{M}_{2} \longrightarrow \mathcal{M}_{1} \longrightarrow M$, where $\mathcal{M}_{i}(i=1,2)$ is a graded subspace of degree less than or equal to $i$. The local coordinates are $\left(x^{i}, q^{a}, p_{a}, \xi_{i}\right)$ of degrees $(0,1,2,3)$. The P-structure $\omega$ is an odd symplectic form of degree 3 , and it can be locally written as

$$
\omega=\delta x^{i} \wedge \delta \xi_{i}-\delta q^{a} \wedge \delta p_{a} .
$$

Since the Q-structure function is of degree 4, its general form is

$$
\Theta=f_{1}{ }^{i} a(x) \xi_{i} q^{a}+\frac{1}{2} f_{2}{ }^{a b}(x) p_{a} p_{b}+\frac{1}{2} f_{3}{ }^{a} b c(x) p_{a} q^{b} q^{c}+\frac{1}{4 !} f_{4 a b c d}(x) q^{a} q^{b} q^{c} q^{d},
$$


where the $f_{i}$ 's are local functions of $x$. The Q-structure condition $\{\Theta, \Theta\}=0$ imposes the following relations on these functions:

$$
\begin{aligned}
& f_{1}{ }^{i} f_{2} f^{b a}=0, \\
& f_{1}{ }^{k}{ }_{c} \frac{\partial f_{2}{ }^{a b}}{\partial x^{k}}+f_{2}{ }^{d a} f_{3}{ }^{b} c d+f_{2}{ }^{d b} f_{3}{ }^{a} c d=0, \\
& f_{1}{ }^{k} b \frac{\partial f_{1}{ }^{i} a}{\partial x^{k}}-f_{1}{ }^{k}{ }_{a} \frac{\partial f_{1}{ }^{i} b}{\partial x^{k}}+f_{1}{ }^{i}{ }_{c} f_{3}{ }^{c} a b=0, \\
& f_{1}{ }^{k}\left[d \frac{\left.\partial f_{3}{ }^{a} b c\right]}{\partial x^{k}}+f_{2}{ }^{a e} f_{4 b c d e}-f_{3}{ }^{a}{ }^{e[b} f_{3}{ }^{e} c d\right]=0, \\
& f_{1}^{k}{ }_{[a} \frac{\partial f_{4 b c d e]}}{\partial x^{k}}+f_{3}{ }_{[a b} f_{4 c d e] f}=0 .
\end{aligned}
$$

Here, $[a b c \cdots]$ is the 'intermolecular antisymmetrization', i.e., for two completely antisymmetric tensors $f_{a_{1} \cdots a_{r}}$ and $g_{b_{1} \cdots b_{s}}$, this is an antisymmetric sum of only nonantisymmetric indices of $f$ and $g$ with unit weight,

$$
f_{\left[a_{1} \cdots a_{r}\right.} g_{\left.b_{1} \cdots b_{s}\right]}=\frac{1}{r ! s !} \sum_{\sigma \in \mathfrak{S}_{r+s}} \operatorname{sgn}(\sigma) f_{a_{\sigma(1)} \cdots a_{\sigma(r)}} g_{a_{\sigma(r+1)} \cdots a_{\sigma(r+s)}} .
$$

For example, $\left.f_{3}{ }^{a}{ }_{e[b} f_{3}{ }^{e} c d\right]=f_{3}{ }^{a}{ }_{e b} f_{3}{ }^{e} c d+f_{3}{ }^{a}{ }_{e c} f_{3}{ }^{e} d b+f_{3}{ }^{a}{ }_{e d} f_{3}{ }^{e} b c$ and $f_{3}{ }^{f}{ }_{[a b} f_{4 c d e] f}$ has $\frac{5 !}{2 ! 3 !}=10$ terms. $\left.\right|^{n}$

These identities, equations (5.67) -(5.70), define the Lie 3-algebroid on the vector bundle $E$, also called the Lie algebroid up to homotopy, or the splittable H-twisted Lie algebroid [81]. It is a special case of the H-twisted Lie algebroid [56].

\subsubsection{Higher Dorfman Bracket}

Let $E$ be a vector bundle on $M$, and let $\mathcal{M}=T^{*}[n] E[1]$ be a graded manifold of degree $n$, where $n \geq 4$. We take local coordinates $\left(x^{i}, q^{a}, p_{a}, \xi_{i}\right)$ of degrees $(0,1, n-1, n)$. The QPstructure is naturally defined on $\mathcal{M}=T^{*}[n] E[1]$, and the P-structure $\omega$ is of degree $n$ and can be locally written as

$$
\omega=\delta x^{i} \wedge \delta \xi_{i}+(-1)^{n|q|} \delta q^{a} \wedge \delta p_{a}
$$

$\bar{n}$ If we take the notation that $[--]$ denotes complete antisymmetrization, equation $(5.70)$ is $f_{1}^{k}\left[d \frac{\left.\partial f_{3}{ }^{a} b c\right]}{\partial x^{k}}+\right.$ $2 f_{2}{ }^{a e} f_{4 b c d e}-f_{3}{ }^{a}{ }_{e[b} f_{3}{ }^{e}{ }_{c d]}=0$, and equation (5.70) is $f_{1}{ }^{k}{ }_{[a} \frac{\partial f_{4 b c d e]}}{\partial x^{k}}+\frac{1}{2} f_{3}{ }_{[a b} f_{4 c d e] f}=0$. 
The general form of the Q-structure function is of degree $n+1$, and we have

$$
\Theta=f_{1{ }^{i} a}(x) \xi_{i} q^{a}+\frac{1}{2} f_{2}{ }^{a} b c(x) p_{a} q^{b} q^{c}+\frac{1}{(n+1) !} f_{3 a_{1} \cdots a_{n+1}}(x) q^{a_{1}} q^{a_{2}} \cdots q^{a_{n+1}},
$$

where the $f_{i}$ 's are functions. The Q-structure condition $\{\Theta, \Theta\}=0$ imposes the following relations: ${ }^{o}$

$$
\begin{aligned}
& f_{1}{ }^{k}{ }_{b} \frac{\partial f_{1}{ }^{i} a}{\partial x^{k}}-f_{1}{ }^{k}{ }_{a} \frac{\partial f_{1}{ }^{i} b}{\partial x^{k}}+f_{1}{ }^{i}{ }_{c} f_{2}{ }^{c} a b=0, \\
& \left.f_{1}{ }^{k}{ }_{[d} \frac{\left.\partial f_{2}{ }^{a} b c\right]}{\partial x^{k}}-f_{2}{ }^{a}{ }_{e[b} f_{2}{ }^{e} c d\right]=0, \\
& f_{1}{ }^{k}\left[a_{1} \frac{\partial f_{\left.3 a_{2} \cdots a_{n+2}\right]}}{\partial x^{k}}+f_{2}{ }^{f}{ }_{\left[a_{1} a_{2}\right.} f_{\left.3 a_{3} \cdots a_{n+2}\right] f}=0 .\right.
\end{aligned}
$$

A vector bundle $E \oplus \wedge^{n-1} E^{*}$ is naturally embedded into $T^{*}[n] E[1]$ by degree shifting. The QP-structure induces an algebroid structure on $E \oplus \wedge^{n-1} E^{*}$ by the derived bracket $[-,-]_{C D}=$ $\{\{-, \Theta\},-\}$, which is called the higher Dorfman bracket. It has the following form:

$$
[u+\alpha, v+\beta]_{C D}=[u, v]+L_{u} \beta-\iota_{v} d \alpha+H(u, v)
$$

where $u, v \in \Gamma(E) ; \alpha, \beta \in \Gamma\left(\wedge^{n-1} E^{*}\right)$; and $H$ is a closed $(n+1)$-form on $E$. We refer to Refs. [58, 148, 15, 152, for detailed studies on brackets of this type. The graded manifold was analyzed in Ref. [152].

\subsubsection{Nonassociative Example}

A large class of nontrivial nonassociative algebras (algebroids) are included in a QP-manifold of degree $n$, and we show one such example. We define $\Theta$ as

$$
\Theta=\Theta_{0}+\Theta_{2}+\Theta_{3}+\cdots+\Theta_{n}
$$

where

$$
\Theta_{0}=f_{0}^{a(0)}{ }_{b(1)}(x) \xi_{a(0)} q^{b(1)}
$$

and

$$
\Theta_{i}=\frac{1}{i !} f_{i a(n-i+1) b_{1}(1) \cdots b_{i}(1)}(x) q^{a(n-i+1)} q^{b_{1}(1)} \cdots q^{b_{i}(1)},
$$

${ }^{o}$ In a complete-antisymmetrization notation, equation (5.76) is $f_{1}{ }^{k}{ }_{\left[a_{1}\right.} \frac{\partial f_{\left.3 a_{2} \cdots a_{n+2}\right]}}{\partial x^{k}}+\frac{2}{n+1} f_{2}{ }^{f}{ }_{\left[a_{1} a_{2}\right.} f_{\left.3 a_{3} \cdots a_{n+2}\right] f}=$ 0 . 
where $i=2,3, \cdots n$, and $\left(x^{a(0)}, q^{a_{1}(1)}, \cdots, q^{a(n-1)}, \xi_{a(0)}\right)$ have degrees $(0,1, \cdots, n-1, n)$. In particular, $\Theta_{n}$ is an $(n+1)$-form on $\Gamma\left(\bigwedge^{n+1} E_{1}\right)$. Then, the master equation $\{\Theta, \Theta\}=0$ is equivalent to

$$
\begin{aligned}
& \left\{\Theta_{0}, \Theta_{0}\right\}=0, \\
& \left\{\Theta_{0}, \Theta_{i}\right\}=0, \quad i<n,
\end{aligned}
$$

and

$$
\begin{aligned}
\left\{\Theta_{0}, \Theta_{n}\right\}+\sum\left\{\Theta_{i}, \Theta_{n-i}\right\} & =0, \quad(n \text { odd }), \\
\left\{\Theta_{0}, \Theta_{n}\right\}+\frac{1}{2}\left\{\Theta_{n / 2}, \Theta_{n / 2}\right\}+\sum\left\{\Theta_{i}, \Theta_{n-i}\right\} & =0, \quad(n \text { even }) .
\end{aligned}
$$

The first condition (5.81) implies that $d:=\left\{\Theta_{0},-\right\}$ is a differential, and the second one (5.82) implies that $\Theta_{i}$ is a closed $i$-form for each $i<n$. The third condition (5.83) says that $\Theta_{n}$ is a closed $(n+1)$-form up to homotopy [142]. This structure is regarded as an $n$-term $L_{\infty}$-algebra.

\section{AKSZ Construction of Topological Field Theories}

In this section, the superfield formalism of topological field theories presented in Sections 2 and 3 is reformulated by the AKSZ construction. If a QP-structure on the target graded manifold $\mathcal{M}$ is given, a QP-structure is induced on the mapping space (i.e., a space of fields) from the world-volume graded manifold $\mathcal{X}$ to the target graded manifold $\mathcal{M}$. [5, 35, 124]

Let $(\mathcal{X}, D)$ be a differential graded manifold (a dg manifold) $\mathcal{X}$ with a $D$-invariant nondegenerate measure $\mu$, where $D$ is a differential on $\mathcal{X}$. Let $(\mathcal{M}, \omega, Q)$ be a QP-manifold of degree $n$, where $\omega$ is a graded symplectic form of degree $n$ and $Q=\{\Theta,-\}$ is a differential on $\mathcal{M}$. $\operatorname{Map}(\mathcal{X}, \mathcal{M})$ is a space of smooth maps from $\mathcal{X}$ to $\mathcal{M}$. The QP-structure on $\operatorname{Map}(\mathcal{X}, \mathcal{M})$ is constructed from the above data.

Since $\operatorname{Diff}(\mathcal{X}) \times \operatorname{Diff}(\mathcal{M})$ naturally acts on $\operatorname{Map}(\mathcal{X}, \mathcal{M}), D$ and $Q$ induce differentials on $\operatorname{Map}(\mathcal{X}, \mathcal{M}), \hat{D}$ and $\check{Q}$. Explicitly, $\hat{D}(z, f)=D(z) d f(z)$ and $\check{Q}(z, f)=Q f(z)$, for $z \in \mathcal{X}$ and $f \in \mathcal{M}^{\mathcal{X}}=\operatorname{Map}(\mathcal{X}, \mathcal{M})$.

Now, we introduce the following two maps. The evaluation map ev : $\mathcal{X} \times \mathcal{M}^{\mathcal{X}} \longrightarrow \mathcal{M}$ is defined as

$$
\mathrm{ev}:(z, f) \longmapsto f(z)
$$


where $z \in \mathcal{X}$ and $f \in \mathcal{M}^{\mathcal{X}}$.

The chain map on the space of graded differential forms, $\mu_{*}: \Omega^{\bullet}\left(\mathcal{X} \times \mathcal{M}^{\mathcal{X}}\right) \longrightarrow \Omega^{\bullet}\left(\mathcal{M}^{\mathcal{X}}\right)$, is defined as

$$
\mu_{*} \omega(f)\left(v_{1}, \ldots, v_{k}\right)=\int_{\mathcal{X}} \mu(z) \omega(z, f)\left(v_{1}, \ldots, v_{k}\right),
$$

for a graded differential form $\omega$, where $v$ is a vector field on $\mathcal{X}$, and $\int_{\mathcal{X}} \mu$ is the integration over $\mathcal{X}$. When the degree is even, the integral is the standard one, but when the degree is odd, it is the Berezin integral. The map $\mu_{*} \mathrm{ev}^{*}: \Omega^{\bullet}(\mathcal{M}) \longrightarrow \Omega^{\bullet}\left(\mathcal{M}^{\mathcal{X}}\right)$, which is called the transgression map, maps a graded differential form on the target space to a graded differential form on the mapping space.

The $P$-structure on $\operatorname{Map}(\mathcal{X}, \mathcal{M})$ is defined as follows:

Definition 6.1 For a graded symplectic form $\omega$ on $\mathcal{M}$, a graded symplectic form $\boldsymbol{\omega}$ on $\operatorname{Map}(\mathcal{X}, \mathcal{M})$ is defined as $\boldsymbol{\omega}:=\mu_{*} \mathrm{ev}^{*} \omega$.

Here, $\boldsymbol{\omega}$ is nondegenerate and closed, because $\mu_{*} \mathrm{ev}^{*}$ preserves nondegeneracy and closedness. Also, $\boldsymbol{\omega}$ is a graded symplectic form on $\operatorname{Map}(\mathcal{X}, \mathcal{M})$ and induces a graded Poisson bracket $\{-,-\}$, which is a $\mathrm{BV}$ antibracket on $\operatorname{Map}(\mathcal{X}, \mathcal{M})$.

Next, the $Q$-structure $S$ on $\operatorname{Map}(\mathcal{X}, \mathcal{M})$ is constructed. $S$ corresponds to a $B V$ action and consists of two parts: $S=S_{0}+S_{1}$. We take a canonical 1-form (the Liouville 1-form) $\vartheta$ for the P-structure on $\mathcal{M}$ such that $\omega=-\delta \vartheta$, and we define $S_{0}:=\iota_{\hat{D}} \mu_{*} \mathrm{ev}^{*} \vartheta$, which is equal to the kinetic term of the BF theory $S^{(0)}$ presented in Section $2 p S_{1}$ is constructed as follows: We take the Q-structure $\Theta$ on $\mathcal{M}$ and map it by the transgression map, $S_{1}:=\mu_{*} \mathrm{ev}^{*} \Theta$.

From the definitions of $S_{0}$ and $S_{1}$, we can prove that $S$ is a Q-structure on $\operatorname{Map}(\mathcal{X}, \mathcal{M})$ [35]:

$$
\{\Theta, \Theta\}=0 \Longleftrightarrow\{S, S\}=0 .
$$

The right-hand side of this equation is the classical master equation in the BV formalism. The homological vector field $\boldsymbol{Q}$ on the mapping space is defined as $\boldsymbol{Q}=\{S,-\}$. By counting the degrees of $\{-,-\}$ and $S$, it can be seen that the degree of $\boldsymbol{Q}$ is 1 . $Q$ is a coboundary operator, $\boldsymbol{Q}^{2}=0$, by the classical master equation. The cohomology defined by $\boldsymbol{Q}$ is called BRST cohomology. Since $\left\{S_{0}, S_{0}\right\}=0, S_{0}$ is considered to be a differential, and $S_{1}$ is ${ }^{p}$ In the remainder of this paper, $S^{(0)}$ will be denoted as $S_{0}$. 
considered to be a connection. The classical master equation $\{S, S\}=2 \delta_{0} S_{1}+\left\{S_{1}, S_{1}\right\}=0$ is regarded as flatness condition, i.e., Maurer-Cartan equation.

The following theorem has been proved. [5]

Theorem 6.2 If $\mathcal{X}$ is a differential graded manifold with a compatible measure and $\mathcal{M}$ is a QP-manifold, then the graded manifold $\operatorname{Map}(\mathcal{X}, \mathcal{M})$ inherits a QP-structure.

In fact, the $\mathrm{QP}$-structure on $\operatorname{Map}(\mathcal{X}, \mathcal{M})$ yields a topological field theory.

A topological field theory constructed from the BV formalism is derived by considering a special super-world-volume $\mathcal{X}$. Let $X$ be an $(n+1)$-dimensional smooth manifold. The supermanifold $\mathcal{X}=T[1] X$ has a Berezin measure $\mu$ of degree $-n-1$, which is induced by the measure on $X$. We can prove that the topological field theories in the previous sections can be constructed by the AKSZ construction on $T[1] X$. Conversely, if $\mathcal{X}=T[1] X$, a QP-structure on $\operatorname{Map}(\mathcal{X}, \mathcal{M})$ is equivalent to the BV formalism of a topological field theory [35, 71]. We can prove that this theory is gauge invariant and unitary by physical arguments, thus it defines a consistent quantum field theory.

Definition 6.3 An AKSZ sigma model (AKSZ topological field theory) in $n+1$ dimensions is a QP-structure constructed in Theorem 6.2, where $X$ in $\mathcal{X}=T[1] X$ is an $n+1$ dimensional manifold and $\mathcal{M}$ is a QP-manifold of degree $n$.

In an $\mathrm{AKSZ}$ sigma model, $\operatorname{Map}(\mathcal{X}, \mathcal{M})$ is a $\mathrm{QP}$-manifold of degree -1 , since there is a measure of degree $-n-1$ on $\mathcal{X}$ and a $\mathrm{QP}$-structure on $\mathcal{M}$ of degree $n$. Therefore, it is an odd symplectic manifold. The graded Poisson bracket $\{-,-\}$ is of degree 1 and $S$ is of degree 0 .

The AKSZ formalism can be applied to realize the Batalin-Fradkin-Vilkovisky (BFV) formalism corresponding to the Hamiltonian formalism, if we choose an $n$-dimensional manifold $X$ and $\mathcal{X}$ has a measure of degree $-n$. [42] Then, the AKSZ construction defines a QPstructure of degree 0 on $\operatorname{Map}(\mathcal{X}, \mathcal{M})$. Its P-structure is the usual Poisson bracket and $\Theta$ is the BRST charge of the BFV formalism.

In order to quantize the theory by the BV formalism, the classical master equation (6.84) must be modified to the quantum master equation. An odd Laplace operator $\Delta$ on $\operatorname{Map}(\mathcal{X}, \mathcal{M})$ can be constructed if $\operatorname{Map}(\mathcal{X}, \mathcal{M})$ has a measure $\boldsymbol{\rho}$. [87, 88, 89] It is defined as

$$
\Delta F=\frac{(-1)^{|F|}}{2} \operatorname{div} \boldsymbol{\rho} X_{F},
$$


where $F \in C^{\infty}(\operatorname{Map}(\mathcal{X}, \mathcal{M}))$ and $X_{F}$ is the Hamiltonian vector field of $F$. Here, the divergence div of the vector field $X$ is defined as $\int_{\mathcal{M}} \boldsymbol{\rho}\left(\operatorname{div}_{\rho} X\right) F=-\int_{\mathcal{M}} \boldsymbol{\rho} X(F)$ for arbitrary $F \in C^{\infty}(\operatorname{Map}(\mathcal{X}, \mathcal{M}))$. If an odd Laplace operator is given, an odd Poisson bracket can be constructed by the derived bracket:

$$
\begin{aligned}
\{F, G\}: & =(-1)^{|F|}[[\Delta, F], G](1) \\
& =(-1)^{|F|} \Delta(F G)-(-1)^{|F|} \Delta(F) G-F \Delta(G)
\end{aligned}
$$

The classical master equation is modified to the following equation:

$$
\Delta\left(e^{\frac{i}{\hbar} S_{q}}\right)=0
$$

where $S_{q}$ is the quantum BV action, which is a deformation of a classical BV action $S_{q}=$ $S+\cdots$. This equation is equivalent to the quantum master equation:

$$
-2 i \hbar \Delta S_{q}+\left\{S_{q}, S_{q}\right\}=0
$$

The above definition of the odd Laplace operator $\Delta$ is formal, because $\operatorname{Map}(\mathcal{X}, \mathcal{M})$ is infinite dimensional in general. The naive measure $\boldsymbol{\rho}$ is divergent and needs regularization. Moreover, even if the graded manifold is finite dimensional, the solutions of the quantum master equation have obstructions, that depend on the topological properties of the base manifold. We refer to Refs. [21, 19, 37] for analyses of the obstructions of the quantum master equation related to the odd Laplace operator in AKSZ theories.

\section{Deformation Theory}

In this section, we apply the deformation theory to the AKSZ formalism of TFTs and determine the most general consistent local BV action $S$ under physical conditions. This method is also called homological perturbation theory.

We begin with $S=S_{0}$. In fact, $S_{0}=S^{(0)}$ is determined from the P-structure only, and it trivially satisfies the classical master equation $\left\{S_{0}, S_{0}\right\}=0$. Next, we deform $S_{0}$ to

$$
S=\sum_{n=0}^{\infty} g^{n} S^{(n)}=S^{(0)}+g S^{(1)}+g^{2} S^{(2)}+\cdots
$$


in order to obtain a consistent $S_{1}$ term, where $g$ is the deformation parameter. $S$ is required to satisfy the classical master equation $\{S, S\}=0$ in order to be a Q-structure.

The deformation $S^{\prime}$ is equivalent to $S$ if there exist local redefinitions of superfields $\boldsymbol{e}^{a(i)} \mapsto$ $\boldsymbol{e}^{a(i)}=F\left(\boldsymbol{e}^{a(i)}\right)$ satisfying $S^{\prime}\left(\boldsymbol{e}^{a(i)}\right)=S\left(\boldsymbol{e}^{a(i)}\right)$, where $F$ is a function on $\operatorname{Map}(\mathcal{X}, \mathcal{M})$. If we expand $\boldsymbol{e}^{a(i)}=\sum_{m} g^{m} F^{(m)}\left(\boldsymbol{e}^{a(i)}\right)$, then $S\left(\boldsymbol{e}^{a(i)}\right)=S^{\prime}\left(\boldsymbol{e}^{a(i)}\right)=S^{\prime}\left(\sum_{m} g^{m} F^{(m)}\left(\boldsymbol{e}^{a(i)}\right)\right)=$ $S^{\prime}\left(\boldsymbol{e}^{a(i)}\right)+g \frac{\delta S^{\prime}\left(\boldsymbol{e}^{a(i)}\right)}{\delta \boldsymbol{e}^{b(j)}} F^{(1)}\left(\boldsymbol{e}^{b(j)}\right)+\cdots$. Therefore, the difference between the two actions is BRST exact to first order in $g$ :

$$
S^{\prime}-S= \pm g \boldsymbol{Q}^{\prime}\left(\int \operatorname{de} F^{(1)}\right),
$$

where $\boldsymbol{Q}^{\prime}$ is the BRST transformation defined by $S^{\prime}$. It has been proved that higher-order terms can be absorbed order by order by the BRST exact terms. Therefore, $S$ is equivalent to $S_{0}$ by field redefinition if the deformation is exact $S=S_{0}+\delta(*)$. Therefore, computing the $\boldsymbol{Q}$ cohomology class is sufficient for determining $S$.

If we substitute equation (7.87) into $\{S, S\}=0$ and expand it in $g$, we obtain the following series of equations:

$$
\begin{aligned}
& \left\{S^{(0)}, S^{(0)}\right\}=0 \\
& \left\{S^{(0)}, S^{(1)}\right\}=0 \\
& 2\left\{S^{(0)}, S^{(2)}\right\}+\left\{S^{(1)}, S^{(1)}\right\}=0 \\
& \cdots
\end{aligned}
$$

The first equation is already satisfied by construction. The second equation is $\boldsymbol{Q}_{0} S^{(1)}=0$. Therefore, $S^{(1)}$ is a cocycle of $\boldsymbol{Q}_{0}$.

The third equation is an obstruction. We assume that the action is local. Thus, $S^{(1)}$ and $S^{(2)}$ are integrals of local Lagrangians. This means that it is the transgression of a function $\Theta^{(2)}$ on the target space, $S^{(2)}=\mu_{*} \operatorname{ev}^{*} \Theta^{(2)}$, where $\Theta^{(2)} \in C^{\infty}(\mathcal{M})$. Since $\left\{S_{0}, \boldsymbol{e}^{a(i)}\right\}=\boldsymbol{d} \boldsymbol{e}^{a(i)}$ for all superfields $\boldsymbol{e}^{a(i)},\left\{S^{(0)}, S^{(2)}\right\}=\boldsymbol{Q}_{0} S^{(2)}=0$, provided the integral of the total derivative terms vanishes, $\int_{\mathcal{X}} \mu \boldsymbol{d}(*)=0$. Therefore, if we assume that $\mathcal{X}$ has no boundary, each term must be equal to zero: $\left\{S^{(0)}, S^{(2)}\right\}=0,\left\{S^{(1)}, S^{(1)}\right\}=0$.

From $\left\{S^{(0)}, S^{(2)}\right\}=0$, we can absorb $S^{(2)}$ into $S^{(1)}$ by the following redefinition: $\tilde{S}^{(1)}=$ $S^{(1)}+g S^{(2)}$. Then, we have $\left\{S^{(0)}, \tilde{S}^{(1)}\right\}=0$. Repeating this process, we obtain $S=S_{0}+S_{1}$, where $S_{1}=\sum_{n=1}^{\infty} g^{n} S^{(n)}$. Here, $S_{1}$ is an element of the cohomology class of $\boldsymbol{Q}_{0}$, 
Lemma 7.1 Denote $S_{1}=\int_{\mathcal{X}} \mu \mathcal{L}_{1}$. If $\mathcal{L}_{1}$ contains a superderivative $\boldsymbol{d}$, then $\mathcal{L}_{1}$ is $\boldsymbol{Q}_{0}$-exact.

Proof It is sufficient to prove the lemma under the assumption that $\mathcal{L}_{1}$ is a monomial. Assume that $\mathcal{L}_{1}$ contains at least one derivative, $\mathcal{L}_{1}(\boldsymbol{e})=F(\boldsymbol{e}) \boldsymbol{d} G(\boldsymbol{e})$, where $F(\boldsymbol{e})$ and $G(\boldsymbol{e})$ are functions of superfields. $F$ and $G$ can be expanded in component superfields by the number of odd supercoordinates $\theta^{\mu}$ as $F(\boldsymbol{e})=\sum_{i=0}^{n+1} F_{i}$ and $G(\boldsymbol{e})=\sum_{i=0}^{n+1} G_{i} . F_{i}$ and $G_{i}$ are terms of $i$-th order in $\theta^{\mu}$. Since $\boldsymbol{Q}_{0} F=\boldsymbol{d} F$ and $\boldsymbol{Q}_{0} G=\boldsymbol{d} G$, from the properties of $\boldsymbol{Q}_{0}$, we obtain the following expansions:

$$
\begin{aligned}
& \boldsymbol{Q}_{0} F_{0}=0, \\
& \boldsymbol{Q}_{0} F_{i}=\boldsymbol{d} F_{i-1} \quad \text { for } 1 \leq i \leq n+1, \\
& \boldsymbol{d} F_{n+1}=0, \\
& \boldsymbol{Q}_{0} G_{0}=0, \\
& \boldsymbol{Q}_{0} G_{i}=\boldsymbol{d} G_{i-1} \quad \text { for } 1 \leq i \leq n+1, \\
& \boldsymbol{d} G_{n+1}=0 .
\end{aligned}
$$

For $S_{1}=\int_{\mathcal{X}} \mu \mathcal{L}_{1}(\boldsymbol{e})=\sum_{i=0}^{n} \int_{X} \mu F_{n-i} \boldsymbol{d} G_{i}$, two consecutive terms $F_{n-i} \boldsymbol{d} G_{i}+F_{n-i-1} \boldsymbol{d} G_{i+1}$ are combined (for even $i$ ) as

$$
F_{n-i} \boldsymbol{d} G_{i}+F_{n-i-1} \boldsymbol{d} G_{i+1}=(-1)^{n-i} \boldsymbol{Q}_{0}\left(F_{n-i} G_{i+1}\right)-(-1)^{n-i} \boldsymbol{d}\left(F_{n-i-1} G_{i+1}\right),
$$

by (7.90), which gives a $\boldsymbol{Q}_{0}$-exact term up to a $\boldsymbol{d}$-exact term.

If $n$ is odd, $S_{1}=\sum_{i=0}^{n} \int_{X} F_{n-i-1} \boldsymbol{d} G_{i}$ has an even number of terms, and the terms can be combined as in equation (7.91). Therefore, the integral $S_{1}$ is $\boldsymbol{Q}_{0}$-exact.

If $n$ is even, the term $F_{0} \boldsymbol{d} G_{n}$ remains. This term is $\boldsymbol{Q}_{0}$-exact itself, since $F_{0} \boldsymbol{d} G_{n}=$ $\boldsymbol{Q}_{0}\left(F_{0} G_{n+1}\right)$. Therefore, $S_{1}$ is also $\boldsymbol{Q}_{0}$-exact.

From Lemma 7.1, nontrivial deformation terms of $S_{1}$ do not include $\boldsymbol{d}$. The remaining condition is $\left\{S_{1}, S_{1}\right\}=0$. Therefore, the following theorem has been proved.

Theorem 7.2 Assume that $\mathcal{X}$ is a world-volume without boundary, that is, $\int_{\mathcal{X}} \mu \boldsymbol{d}(*)=$ 0 , and locality of the $B V$ action. If and only if $S^{(1)}$ is a $\boldsymbol{Q}_{0}$-cohomology class such that $\left\{S^{(1)}, S^{(1)}\right\}=0$, and $S^{(n)}=0$ for $n \geq 2$, then $\{S, S\}=0$. Let $S_{1}=g S^{(1)}=\int_{\mathcal{X}} \mu \mathcal{L}_{1}(\boldsymbol{e})$, then $\mathcal{L}_{1}(\boldsymbol{e})$ is a function of a superfield $\boldsymbol{e}$, which does not contain the superderivative $\boldsymbol{d}$. 
If we relax the assumption of no-boundary or locality in Theorem [7.2, we obtain more general AKSZ type sigma models, such as the WZ-Poisson sigma model and the Dirac sigma model. [98]

\section{AKSZ Sigma Models in Local Coordinates}

In this section, we give local coordinate expressions of the P-structure graded symplectic form $\boldsymbol{\omega}$, the BV antibracket, the BV action $S$, (17.87) and the odd Laplacian in the previous section.

Let us take an $(n+1)$-dimensional manifold $X$ and a $d$-dimensional manifold $M$. We also take a graded manifold $\mathcal{X}=T[1] X$, and a QP-manifold $\mathcal{M}$. Local coordinates on $T[1] X$ are denoted by $\left(\sigma^{\mu}, \theta^{\mu}\right)$, where $\sigma^{\mu}$ is a local coordinate of degree 0 on the base manifold $X$, and $\theta^{\mu}$ is a local coordinate of degree 1.

Let $\mathcal{M}^{(i)}$ be the degree $i$ part of $\mathcal{M}$. Local coordinates on $\mathcal{M}^{(i)}$ are denoted by $e^{a(i)}$. The local coordinates $e^{a(i)}$ are also denoted by

1. $x^{a(0)}$ of degree 0

2. $q^{a(i)}$ of degree $i$, for $0 \leq i \leq\lfloor n / 2\rfloor$

3. $p_{a(n-i)}$ of degree $n-i$, for $\lfloor n / 2\rfloor<i \leq n$

4. $\xi_{a(0)}$ of degree $n$

where $\lfloor m\rfloor$ is the floor function (that is, its value is the largest integer less than or equal to $m) . q$

As explained in Section 6, fields in a classical field theory correspond to maps $\mathcal{X} \rightarrow \mathcal{M}$. Local coordinates on the mapping space are superfields, which we denote by the corresponding boldface letters. $\boldsymbol{x}^{a(0)}$ of degree 0 is a smooth map $\boldsymbol{x}^{a(0)}: T[1] X \longrightarrow M$, and superfields $\boldsymbol{e}^{a(i)}$ of degree $i$ are bases of sections of $T^{*}[1] X \otimes \boldsymbol{x}^{*}\left(\mathcal{M}^{(i)}\right)$, for $1 \leq i \leq n$. $\boldsymbol{x}^{a(0)}$ is also denoted by $\boldsymbol{e}^{a(0)}$ and $\boldsymbol{e}^{a(n)}$ by $\boldsymbol{\xi}_{a(0)}$.

${ }^{q}$ Indices $a(i) \operatorname{run} a(i)=1,2, \cdots, \operatorname{dim} \mathcal{M}^{(i)}$. 
The P-structure can be written as

$$
\begin{aligned}
\boldsymbol{\omega} & =\int_{\mathcal{X}} \mu\left(\frac{1}{2} \delta \boldsymbol{e}_{a(i)} \wedge \boldsymbol{\omega}^{a(i) b(j)} \delta \boldsymbol{e}_{b(j)}\right) \\
& =\sum_{i=0}^{\lfloor n / 2\rfloor} \int_{\mathcal{X}} d^{n+1} \sigma d^{n+1} \theta(-1)^{n i} \delta \boldsymbol{q}^{a(i)} \wedge \delta \boldsymbol{p}_{a(i)},
\end{aligned}
$$

where we used Darboux coordinates, $\boldsymbol{q}^{a(i)}$, for $0 \leq i \leq\lfloor n / 2\rfloor$, and $\boldsymbol{p}_{a(n-i)}$, for $\lfloor n / 2\rfloor<i \leq n$. This defines the graded Poisson bracket such that

$$
\left\{\boldsymbol{q}^{a(i)}(\sigma, \theta), \boldsymbol{p}_{b(j)}\left(\sigma^{\prime}, \theta^{\prime}\right)\right\}=\delta_{j}^{i} \delta^{a(i)} b(j) \delta^{n+1}\left(\sigma-\sigma^{\prime}\right) \delta^{n+1}\left(\theta-\theta^{\prime}\right) .
$$

If $n$ is even, $\boldsymbol{p}_{a(n / 2)}$ is identified with $k_{a b} \boldsymbol{q}^{b(n / 2)}$ and the degree $(n / 2)$ part of the P-structure symplectic form can be written as

$$
\int_{\mathcal{X}} d^{n+1} \sigma d^{n+1} \theta\left(\frac{1}{2} \delta \boldsymbol{q}^{a(n / 2)} \wedge k_{a b} \delta \boldsymbol{q}^{b(n / 2)}\right),
$$

where $k_{a b}$ is a fiber metric. The corresponding Poisson bracket of the part, for which $i=j=$ $n / 2$, is

$$
\left\{\boldsymbol{q}^{a(n / 2)}(\sigma, \theta), \boldsymbol{q}^{b(n / 2)}\left(\sigma^{\prime}, \theta^{\prime}\right)\right\}=k^{a(n / 2) b(n / 2)} \delta^{n+1}\left(\sigma-\sigma^{\prime}\right) \delta^{n+1}\left(\theta-\theta^{\prime}\right) .
$$

The differential $D$ on the differential graded manifold $\mathcal{X}$ is induced from the exterior derivative $d$ on $X$. This defines a superdifferential $\boldsymbol{d}=\theta^{\mu} \frac{\partial}{\partial \sigma^{\mu}}$ on $\operatorname{Map}(\mathcal{X}, \mathcal{M})$.

Next, let us consider the local coordinate expression of the Q-structure $S$ on the mapping space. From the definition in Section [6, $S$ has two terms, $S=S_{0}+S_{1}$.

$S_{0}$ is determined from the P-structure. If $n$ is odd,

$$
\begin{aligned}
S_{0} & =\int_{\mathcal{X}} \mu \frac{1}{2} \boldsymbol{e}_{a(i)} \boldsymbol{\omega}^{a(i) b(j)} \boldsymbol{d} \boldsymbol{e}_{b(j)} \\
& =\int_{\mathcal{X}} d^{n+1} \sigma d^{n+1} \theta \sum_{0 \leq i \leq(n-1) / 2}(-1)^{n+1-i} \boldsymbol{p}_{a(i)} \boldsymbol{d} \boldsymbol{q}^{a(i)} \\
& =\int_{\mathcal{X}} d^{n+1} \sigma d^{n+1} \theta\left((-1)^{n+1} \boldsymbol{\xi}_{a(0)} \boldsymbol{d} \boldsymbol{x}^{a(0)}+\sum_{1 \leq i \leq(n-1) / 2}(-1)^{n+1-i} \boldsymbol{p}_{a(i)} \boldsymbol{d} \boldsymbol{q}^{a(i)}\right),
\end{aligned}
$$


and if $n$ is even,

$$
\begin{aligned}
S_{0}= & \int_{\mathcal{X}} \mu \frac{1}{2} \boldsymbol{e}_{a(i)} \omega^{a(i) b(j)} \boldsymbol{d e}_{b(j)} \\
= & \int_{\mathcal{X}} d^{n+1} \sigma d^{n+1} \theta\left(\sum_{0 \leq i \leq(n-2) / 2}(-1)^{n+1-i} \boldsymbol{p}_{a(i)} \boldsymbol{d} \boldsymbol{q}^{a(i)}+(-1)^{\frac{n+1}{2}} k_{a(n / 2) b(n / 2)} \boldsymbol{q}^{a(n / 2)} \boldsymbol{d} \boldsymbol{q}^{b(n / 2)}\right) \\
= & \int_{\mathcal{X}} d^{n+1} \sigma d^{n+1} \theta\left((-1)^{n+1} \boldsymbol{\xi}_{a(0)} \boldsymbol{d} \boldsymbol{x}^{a(0)}+\sum_{1 \leq i \leq(n-2) / 2}(-1)^{n+1-i} \boldsymbol{p}_{a(i)} \boldsymbol{d} \boldsymbol{q}^{a(i)}\right. \\
& \left.\quad+(-1)^{\frac{n+1}{2}} k_{a(n / 2) b(n / 2)} \boldsymbol{q}^{a(n / 2)} \boldsymbol{d} \boldsymbol{q}^{b(n / 2)}\right) .
\end{aligned}
$$

If we define $\boldsymbol{p}_{a(n / 2)} \equiv k_{a(n / 2) b(n / 2)} \boldsymbol{q}^{a(n / 2)}$, then the $S_{0}$ 's for odd and even $n$ can be unified to

$$
\begin{aligned}
S_{0} & =\int_{\mathcal{X}} \mu \frac{1}{2} \boldsymbol{e}_{a(i)} \omega^{a(i) b(j)} \boldsymbol{d e}_{b(j)} \\
& =\int_{\mathcal{X}} d^{n+1} \sigma d^{n+1} \theta\left(\sum_{0 \leq i \leq\lfloor n / 2\rfloor}(-1)^{n+1-i} \boldsymbol{p}_{a(i)} \boldsymbol{d} \boldsymbol{q}^{a(i)}\right) .
\end{aligned}
$$

A superfield of degree $i, \boldsymbol{\Phi}(\sigma, \theta)$, can be expanded by $\theta^{\mu}$ as

$$
\boldsymbol{\Phi}(\sigma, \theta)=\sum_{k} \boldsymbol{\Phi}^{(k)}(\sigma, \theta)=\sum_{k} \frac{1}{k !} \theta^{\mu(1)} \cdots \theta^{\mu(k)} \Phi_{\mu(1) \cdots \mu(k)}^{(k)}(\sigma),
$$

where $\Phi_{\mu(1) \cdots \mu(k)}^{(k)}(\sigma)$ depends only on $\sigma^{\mu}$. Since $\theta^{\mu}$ has degree $1, \Phi_{\mu(1) \cdots \mu(k)}^{(k)}(\sigma)$ has degree $i-k$. This is the same as the ghost number in gauge theory. The fields $\Phi_{\mu(1) \cdots \mu(k)}^{(k)}(\sigma)$ are classified by their ghost numbers. If $\Phi_{\mu(1) \cdots \mu(k)}^{(k)}(\sigma)$ has degree 0 , it is a physical field. In particular, it is a $k$-th order antisymmetric tensor field. If $\Phi_{\mu(1) \cdots \mu(k)}^{(k)}(\sigma)$ has positive degree, it is a ghost field, or it is a ghost for ghosts, etc. If $\Phi_{\mu(1) \cdots \mu(k)}^{(k)}(\sigma)$ has negative degree, it is the Hodge dual of the antifield that is introduced in the BV formalism.

Let us consider expansions of the Darboux coordinate superfields:

$$
\begin{aligned}
\boldsymbol{q}^{a(i)}(\sigma, \theta) & =\sum_{k} \frac{1}{k !} \theta^{\mu(1)} \cdots \theta^{\mu(k)} q_{\mu(1) \cdots \mu(k)}^{(k), a(i)}(\sigma) \\
\boldsymbol{p}_{a(i)}(\sigma, \theta) & =\sum_{k} \frac{1}{k !} \theta^{\mu(1)} \cdots \theta^{\mu(k)} p_{a(i), \mu(1) \cdots \mu(k)}^{(k)}(\sigma) .
\end{aligned}
$$

The antifield for the ghost $q_{\mu(1) \cdots \mu(k)}^{(k), a(i)}(\sigma)$ for $i-k>0$ is $p_{a(i), \mu(1) \cdots \mu(n+1-k)}^{(n+1-k)}(\sigma)$, and the antifield for the ghost $p_{\mu(1) \cdots \mu(k)}^{(k), a(i)}(\sigma)$ for $k-i>0$ is $q_{a(i), \mu(1) \cdots \mu(n+1-k)}^{(n+1-k)}(\sigma)$. We can see that 
this coincides with the BF theory for abelian $i$-form fields that was presented in Section 3. Note that, if $n$ is even, a superfield of degree $i=n / 2$ is a self-conjugate superfield $\boldsymbol{q}_{a(n / 2)}(\sigma, \theta)=\sum_{k, \mu(k)} \frac{1}{k !} \theta^{\mu(1)} \cdots \theta^{\mu(k)} q_{a(n / 2), \mu(1) \cdots \mu(k)}^{(k)}(\sigma)$. The antifield $q_{\mu(1) \cdots \mu(k)}^{(k), a(n / 2)}(\sigma)$ for $k \leq n / 2$ is $q_{\mu(1) \cdots \mu(n+1-k)}^{(n+1-k), a(n / 2)}(\sigma)$, which is contained in the same superfield.

If the component fields of nonzero ghost number are set to zero and the $d \theta$ integration is carried out, we obtain the kinetic term for a BF theory of general $k$-forms:

$$
S_{0}=S_{A}=\sum_{0 \leq i \leq\lfloor n / 2\rfloor} \frac{1}{i !(n-i) !} \int_{\mathcal{X}} d^{n+1} \sigma(-1)^{n+1-i} \epsilon^{\mu(0) \cdots \mu(n)} p_{a(i), \mu(i+1) \cdots \mu(n)}^{(n-i)} \partial_{\mu(i)} q_{\mu(0) \cdots \mu(i-1)}^{(i), a(i)} .
$$

This coincides with the action $S_{A}$ given in Section 3 ,

The interaction term $S_{1}$ was determined in Theorem 7.2 in Section 7 . The local coordinate expression of $S_{1}$ is as follows:

$$
S_{1}=\sum_{\lambda, a(\lambda),|\lambda|=n+1} \int_{\mathcal{X}} \mu\left(f_{\lambda, a\left(\lambda_{1}\right) \cdots a\left(\lambda_{m}\right)}(\boldsymbol{x}) \boldsymbol{e}^{a\left(\lambda_{1}\right)} \boldsymbol{e}^{a\left(\lambda_{2}\right)} \cdots \boldsymbol{e}^{a\left(\lambda_{m}\right)}\right),
$$

where the integrand contains arbitrary functions of superfields of degree $n+1$ without the superderivative. $f_{\lambda, a\left(\lambda_{1}\right) \cdots a\left(\lambda_{m}\right)}(\boldsymbol{x})$ is a local structure function of $\boldsymbol{x}$ and $|\lambda|=\sum_{k} \lambda_{k}$. The consistency condition $\left\{S_{1}, S_{1}\right\}=0$ imposes algebraic conditions on the structure functions $f_{\lambda, a\left(\lambda_{1}\right) \cdots a\left(\lambda_{m}\right)}(\boldsymbol{x})$. Since $S_{1}=\int_{\mathcal{X}} \mu \mathrm{ev}^{*} \Theta$, this consistency condition is equivalent to $\{\Theta, \Theta\}=0$, and determines the mathematical structure on the target space. Thus, by solving $\{\Theta, \Theta\}=0$, we obtain consistent local expressions for the AKSZ sigma models in $n+1$ dimensions.

Finally, we give the expression of the odd Laplace operator, which appears in the quantum BV master equation. Let $\boldsymbol{\rho}=\rho_{v} d^{n+1} \boldsymbol{q} d^{n+1} \boldsymbol{p}$ be a volume form on $\operatorname{Map}(\mathcal{X}, \mathcal{M})$. The odd Laplace operator,

$$
\Delta F=\frac{(-1)^{|F|}}{2} \operatorname{div} \boldsymbol{\rho} X_{F}
$$

can be written as

$$
\Delta=\int_{\mathcal{X}} d^{n+1} \sigma d^{n+1} \theta \sum_{i=0}^{n}(-1)^{i} \frac{\partial}{\partial \boldsymbol{q}^{a(i)}} \frac{\partial}{\partial \boldsymbol{p}_{a(i)}}+\frac{1}{2}\left\{\ln \rho_{v},-\right\}
$$

If we take coordinates such that $\rho_{v}=1$, we obtain the following simple expression:

$$
\Delta=\int_{\mathcal{X}} d^{n+1} \sigma d^{n+1} \theta \sum_{i=0}^{n}(-1)^{i} \frac{\partial}{\partial \boldsymbol{q}^{a(i)}} \frac{\partial}{\partial \boldsymbol{p}_{a(i)}} .
$$




\section{Examples of AKSZ Sigma Models}

In this section, we list some important examples.

\section{$9.1 n=1$}

\subsubsection{The Poisson Sigma Model}

We take $n=1$. In Example 5.2.1 we showed that a QP-structure of degree 1 on $\mathcal{M}=T^{*}[1] M$ is equivalent to a Poisson structure on $M$. Let $X$ be a two-dimensional manifold, and let $\mathcal{X}=T[1] X$. The AKSZ construction defines a TFT on $\operatorname{Map}\left(T[1] X, T^{*}[1] M\right)$.

Let $\boldsymbol{x}^{i}$ be a map from $T[1] X$ to $M$, and let $\boldsymbol{\xi}_{i}$ be a section of $T^{*}[1] X \otimes \boldsymbol{x}^{*}\left(T^{*}[1] M\right)$, which are superfields induced by the local coordinates $\left(x^{i}, \xi_{i}\right)$. Here, we denote the indices $a(0), b(0)$ by $i, j$. The P-structure on $\operatorname{Map}\left(T[1] X, T^{*}[1] M\right)$ is

$$
\boldsymbol{\omega}=\int_{\mathcal{X}} d^{2} \sigma d^{2} \theta \delta \boldsymbol{x}^{i} \wedge \delta \boldsymbol{\xi}_{i}
$$

The BV action (Q-structure) is

$$
S=\int_{\mathcal{X}} d^{2} \sigma d^{2} \theta\left(\boldsymbol{\xi}_{i} \boldsymbol{d} \boldsymbol{x}^{i}+\frac{1}{2} f^{i j}(\boldsymbol{x}) \boldsymbol{\xi}_{i} \boldsymbol{\xi}_{j}\right) .
$$

This action is the superfield BV formalism of the Poisson sigma model, where the superfields are identified with $\boldsymbol{x}^{i}=\boldsymbol{\phi}^{i}$ and $\boldsymbol{\xi}_{i}=\boldsymbol{A}_{i}$. The Q-structure condition is equivalent to equation (5.41) on $f^{i j}(x)$.

Take $M=\mathfrak{g}^{*}$, where $\mathfrak{g}$ is a semi-simple Lie algebra. Then, $\mathcal{M}=T^{*}[1] \mathfrak{g}^{*}$, and the Qstructure reduces to $\Theta=\frac{1}{2} f^{i j}{ }_{k} x^{k} \xi_{i} \xi_{j}$, where $f^{i j}{ }_{k}$ is a structure constant of the Lie algebra. The AKSZ construction yields the BV action

$$
S=\int_{\mathcal{X}} d^{2} \sigma d^{2} \theta\left(\boldsymbol{\xi}_{i} \boldsymbol{d} \boldsymbol{x}^{i}+\frac{1}{2} f^{i j}{ }_{k} \boldsymbol{x}^{k} \boldsymbol{\xi}_{i} \boldsymbol{\xi}_{j}\right),
$$

which is the BV formalism of a nonabelian BF theory in two dimensions.

\subsubsection{B-Model}

Let $X$ be a Riemann surface, and $M$ a complex manifold. Let us consider the supermanifold $\mathcal{X}=T[1] X$ and the QP-manifold $\mathcal{M}=T^{*}[1] T[1] M$ given in Example 5.2.2. This QPmanifold realizes a complex structure. The AKSZ construction for $n=1$ induces a TFT on $\operatorname{Map}\left(T[1] X, T^{*}[1] T[1] M\right)$. 
Let $\boldsymbol{x}$ be $\boldsymbol{x}: T[1] X \longrightarrow M$, let $\boldsymbol{\xi}$ be a section of $T^{*}[1] X \otimes \boldsymbol{x}^{*}\left(T^{*}[1] M\right)$, let $\boldsymbol{q}$ be a section of $T^{*}[1] X \otimes \boldsymbol{x}^{*}(T[1] M)$, and let $\boldsymbol{p}$ be a section of $T^{*}[1] X \otimes \boldsymbol{x}^{*}\left(T^{*}[0] M\right)$. The superfield expression of the P-structure is

$$
\boldsymbol{\omega}=\int_{\mathcal{X}} d^{2} \sigma d^{2} \theta\left(\delta \boldsymbol{x}^{i} \wedge \delta \boldsymbol{\xi}_{i}-\delta \boldsymbol{q}^{i} \wedge \delta \boldsymbol{p}_{i}\right)
$$

The Q-structure BV action is

$$
\begin{aligned}
S_{B} & =\int_{\mathcal{X}} d^{2} \sigma d^{2} \theta\left(\boldsymbol{\xi}_{i} \boldsymbol{d} \boldsymbol{x}^{i}-\boldsymbol{p}_{i} \boldsymbol{d} \boldsymbol{q}^{i}+J^{i}{ }_{j}(\boldsymbol{x}) \boldsymbol{\xi}_{i} \boldsymbol{q}^{j}+\frac{\partial J^{i}{ }_{k}}{\partial \boldsymbol{x}^{j}}(\boldsymbol{x}) \boldsymbol{p}_{i} \boldsymbol{q}^{j} \boldsymbol{q}^{k}\right) \\
& =\int_{\mathcal{X}} d^{2} \sigma d^{2} \theta\left[\left(\boldsymbol{\xi}_{i} \boldsymbol{q}^{i}\right) \boldsymbol{d}\left(\begin{array}{c}
\boldsymbol{x}^{i} \\
\boldsymbol{p}_{i}
\end{array}\right)+\left(\boldsymbol{\xi}_{i} \boldsymbol{q}^{i}\right)\left(\begin{array}{cc}
0 & \frac{1}{2} J^{i}{ }_{j}(\boldsymbol{x}) \\
-\frac{1}{2} J^{j}{ }_{i}(\boldsymbol{x}) & \frac{\partial J^{i}}{\partial \boldsymbol{x}^{j}}(\boldsymbol{x}) \boldsymbol{p}_{i}
\end{array}\right)\left(\begin{array}{l}
\boldsymbol{\xi}_{j} \\
\boldsymbol{q}^{j}
\end{array}\right)\right] .
\end{aligned}
$$

Proper gauge fixing of this action describes the so-called B-model action of a topological string. [5, 80]

\section{$9.2 n=2$}

\subsubsection{The Courant Sigma Model}

We consider the case, where $\mathcal{M}$ is a QP-manifold of degree $n=2$. Here, $\mathcal{M}$ has the Courant algebroid structure, discussed in Example 5.3.1. We take a three-dimensional manifold $X$ and consider $\mathcal{X}=T[1] X$ as the world-volume of the AKSZ sigma model. Let $\boldsymbol{x}^{i}$ be a map from $T[1] X$ to $M=\mathcal{M}^{(0)}, \boldsymbol{\xi}_{i}$ be a section of $T^{*}[1] X \otimes \boldsymbol{x}^{*}\left(\mathcal{M}^{(2)}\right)$ and $\boldsymbol{\eta}^{a}$ be a section of $T^{*}[1] X \otimes \boldsymbol{x}^{*}\left(\mathcal{M}^{(1)}\right) . \quad k_{a b}$ is a fiber metric on $\mathcal{M}^{(1)}$. Here, we denote $a(0), b(0), \cdots$ by $i, j, \cdots$ and $a(1), b(1), \cdots$ by $a, b, \cdots$. The P-structure on $\operatorname{Map}(\mathcal{X}, \mathcal{M})$ is

$$
\boldsymbol{\omega}=\int_{\mathcal{X}} d^{3} \sigma d^{3} \theta\left(\delta \boldsymbol{x}^{i} \wedge \delta \boldsymbol{\xi}_{i}+\frac{1}{2} k_{a b} \delta \boldsymbol{\eta}^{a} \wedge \delta \boldsymbol{\eta}^{b}\right),
$$

and the Q-structure BV action has the following form:

$$
S=\int_{\mathcal{X}} d^{3} \sigma d^{3} \theta\left(-\boldsymbol{\xi}_{i} \boldsymbol{d} \boldsymbol{x}^{i}+\frac{1}{2} k_{a b} \boldsymbol{\eta}^{a} \boldsymbol{d} \boldsymbol{\eta}^{b}+f_{1}^{i}{ }_{a}(\boldsymbol{x}) \boldsymbol{\xi}_{i} \boldsymbol{\eta}^{a}+\frac{1}{3 !} f_{2 a b c}(\boldsymbol{x}) \boldsymbol{\eta}^{a} \boldsymbol{\eta}^{b} \boldsymbol{\eta}^{c}\right) .
$$

This model has the Courant algebroid structure given in Theorem 5.1, and therefore, it is called the Courant sigma model [72, 73, 66, 124].

We can derive the action of the physical fields from equation (9.100) by setting the com-

ponents of the nonzero ghost number to zero: $\boldsymbol{x}^{i}=\boldsymbol{x}^{(0) i}=x^{i}, \boldsymbol{\xi}_{i}=\boldsymbol{\xi}_{i}^{(2)}=\frac{1}{2} \theta^{\mu} \theta^{\nu} \xi_{\mu \nu, i}^{(2)}$ and 
$\boldsymbol{\eta}^{a}=\boldsymbol{\eta}^{(1) a}=\theta^{\mu} \eta_{\mu}^{(1) a}$. Then, we obtain

$$
S=\int_{X}\left(-\xi_{i} \wedge d x^{i}+\frac{1}{2} k_{a b} \eta^{a} \wedge d \eta^{b}+f_{1 a}^{i}(x) \xi_{i} \wedge \eta^{a}+\frac{1}{3 !} f_{2 a b c}(x) \eta^{a} \wedge \eta^{b} \wedge \eta^{c}\right),
$$

where $d$ is the exterior differential on $X, \xi_{i}=\frac{1}{2} d \sigma^{\mu} \wedge d \sigma^{\nu} \xi_{\mu \nu, i}^{(2)}$ and $\eta^{a}=d \sigma^{\mu} \eta_{\mu}^{(1) a}$.

\subsubsection{Chern-Simons Gauge Theory}

In the Courant sigma model, (9.101), if we take $\xi_{i}=0, f_{1 a}^{i}(x)=0$ and $f_{2 a b c}(x)=f_{2 a b c}=$ constant, the action reduces to the Chern-Simons theory:

$$
S=\int_{X}\left(\frac{1}{2} k_{a b} A^{a} \wedge d A^{b}+\frac{1}{3 !} f_{2 a b c} A^{a} \wedge A^{b} \wedge A^{c}\right),
$$

where we denote the 1 -form by $A^{a}=\eta^{a}$. Therefore, the Chern-Simons theory can be obtained by the AKSZ construction.

In fact, the AKSZ construction in three dimensions for a Lie algebra target space yields the Chern-Simons theory. Let $\mathfrak{g}$ be a Lie algebra and let $k_{a b}$ be a metric on $\mathfrak{g}$. If $\mathfrak{g}$ is semisimple, we can take $k_{a b}$ as the Killing metric. Note that $\mathcal{M}=\mathfrak{g}[1]$ has QP-manifold structure of degree 2 , and $M=\{p t\}$. The P-structure is defined as

$$
\omega=\frac{1}{2} k_{a b} \delta \eta^{a} \wedge \delta \eta^{b}
$$

where $a=a(1), b=b(1), \cdots$. The Q-structure is

$$
\Theta=\frac{1}{3 !} f_{a b c} \eta^{a} \eta^{b} \eta^{c}
$$

where $f_{a b c}$ is the structure constant of $\mathfrak{g}$.

Let $X$ be a three-dimensional manifold and $\mathcal{X}=T[1] X$. Then, $\boldsymbol{\eta}^{a}$ is a section of $T^{*}[1] X \otimes$ $\boldsymbol{x}^{*}(\mathfrak{g}[1])$. The AKSZ construction on $\operatorname{Map}(T[1] X, \mathfrak{g}[1])$ yields the P-structure:

$$
\boldsymbol{\omega}=\int_{\mathcal{X}} d^{3} \sigma d^{3} \theta \frac{1}{2} k_{a b} \delta \boldsymbol{\eta}^{a} \wedge \delta \boldsymbol{\eta}^{b}
$$

and the Q-structure function

$$
S=\int_{\mathcal{X}} d^{3} \sigma d^{3} \theta\left(\frac{1}{2} k_{a b} \boldsymbol{\eta}^{a} \boldsymbol{d} \boldsymbol{\eta}^{b}+\frac{1}{3 !} f_{a b c} \boldsymbol{\eta}^{a} \boldsymbol{\eta}^{b} \boldsymbol{\eta}^{c}\right) .
$$

The action satisfies $\{S, S\}=0$. This is the AKSZ sigma model of the action (9.102) for the Chern-Simons theory in three dimensions [5], which coincides with the BV action obtained in Ref. [7]. 


\section{$9.3 n=3$}

\subsubsection{AKSZ Sigma Model in 4 Dimensions}

We take $n=3$. Then, $X$ is a four-dimensional manifold, and $\mathcal{M}$ is the QP-manifold of degree 3 in Example 5.4.1. Let $\boldsymbol{x}^{i}$ be a map from $T[1] X$ to $M=\mathcal{M}^{(0)}$ and $\boldsymbol{\xi}_{i}$ be a section of $T^{*}[1] X \otimes \boldsymbol{x}^{*}\left(\mathcal{M}^{(3)}\right)$. Let $\boldsymbol{q}^{a}$ be a section of $T^{*}[1] X \otimes \boldsymbol{x}^{*}\left(\mathcal{M}^{(1)}\right)$ and $\boldsymbol{p}_{a}$ be a section of $T^{*}[1] X \otimes \boldsymbol{x}^{*}\left(\mathcal{M}^{(2)}\right)$. Here, we denote $a(0), b(0), \cdots$ by $i, j, \cdots$ and $a(1), b(1), \cdots$ by $a, b, \cdots$. Note that $\left(\boldsymbol{x}^{i}, \boldsymbol{\xi}_{i}, \boldsymbol{q}^{a}, \boldsymbol{p}_{a}\right)$ are superfields of degrees $(0,3,1,2)$. The P-structure is

$$
\boldsymbol{\omega}=\int_{\mathcal{X}} d^{4} \sigma d^{4} \theta\left(\delta \boldsymbol{x}^{i} \wedge \delta \boldsymbol{\xi}_{i}-\delta \boldsymbol{q}^{a} \wedge \delta \boldsymbol{p}_{a}\right) .
$$

The Q-structure funciton is

$$
\begin{aligned}
S & =S_{0}+S_{1}, \\
S_{0} & =\int_{\mathcal{X}} d^{4} \sigma d^{4} \theta\left(\boldsymbol{\xi}_{i} \boldsymbol{d} \boldsymbol{x}^{i}-\boldsymbol{p}_{a} \boldsymbol{d} \boldsymbol{q}^{a}\right), \\
S_{1} & =\int_{\mathcal{X}} d^{4} \sigma d^{4} \theta\left(f_{1}{ }^{i} a(\boldsymbol{x}) \boldsymbol{\xi}_{a} \boldsymbol{q}^{i}+\frac{1}{2} f_{2}{ }^{a b}(\boldsymbol{x}) \boldsymbol{p}_{a} \boldsymbol{p}_{b}+\frac{1}{2} f_{3}{ }^{a}{ }_{b c}(\boldsymbol{x}) \boldsymbol{p}_{a} \boldsymbol{q}^{b} \boldsymbol{q}^{c}+\frac{1}{4 !} f_{4 a b c d}(\boldsymbol{x}) \boldsymbol{q}^{a} \boldsymbol{q}^{b} \boldsymbol{q}^{c} \boldsymbol{q}^{d}\right) .
\end{aligned}
$$

This topological sigma model has the structure of a Lie 3-algebroid, which is also called a Lie algebroid up to homotopy or H-twisted Lie algebroid, that appeared in Example 5.4.1. [81, 56]

\subsubsection{Topological Yang-Mills Theory}

We consider a semi-simple Lie algebra $\mathfrak{g}$ and a graded vector bundle $\mathcal{M}=T^{*}[3] \mathfrak{g}[1] \simeq$ $\mathfrak{g}^{*}[2] \oplus \mathfrak{g}[1]$ of degree 3 on a point $M=\{p t\}$. The world-volume supermanifold is $\mathcal{X}=T[1] X$, where $X$ is a four-dimensional manifold. Then, $\boldsymbol{q}^{a}$ is a section of $T^{*}[1] X \otimes \boldsymbol{x}^{*}(\mathfrak{g}[1])$ and $\boldsymbol{p}_{a}$ is a section of $T^{*}[1] X \otimes \boldsymbol{x}^{*}\left(\mathfrak{g}^{*}[2]\right)$, where $a(1)=a, b(1)=b, \cdots$. The P-structure is

$$
\boldsymbol{\omega}=\int_{\mathcal{X}} d^{4} \sigma d^{4} \theta\left(-\delta \boldsymbol{q}^{a} \wedge \delta \boldsymbol{p}_{a}\right) .
$$

The dual space $\mathfrak{g}^{*}$ has the metric $(\cdot, \cdot)_{K^{-1}}$, which is the inverse of the Killing form on $\mathfrak{g}$. We can define the Q-structure

$$
\Theta=k^{a b} p_{a} p_{b}+\frac{1}{2} f^{a}{ }_{b c} p_{a} q^{b} q^{c}
$$


where $q^{a}$ is a coordinate on $\mathfrak{g}[1], p_{a}$ is a coordinate on $\mathfrak{g}^{*}[2], k^{a b} p_{a} p_{b}:=\left(p_{a}, p_{b}\right)_{K^{-1}}$ and $f_{b c}^{a}$ is the structure constant of the Lie algebra $\mathfrak{g}$. The AKSZ construction determines the following BV action:

$$
S=\int_{\mathcal{X}} d^{4} \sigma d^{4} \theta\left(-\boldsymbol{p}_{a} \boldsymbol{F}^{a}+k^{a b} \boldsymbol{p}_{a} \boldsymbol{p}_{b}\right)
$$

where $\boldsymbol{F}^{a}=\boldsymbol{d} \boldsymbol{q}^{a}-\frac{1}{2} f_{b c}^{a} \boldsymbol{q}^{b} \boldsymbol{q}^{c}$. This derives a topological Yang-Mills theory, if we integrate out $\boldsymbol{p}_{a}$ and make a proper gauge fixing of the remaining superfields. [75]

\subsection{General $n$}

\subsubsection{Nonabelian BF Theories in $n+1$ Dimensions}

Let $n \geq 2$, and let $\mathfrak{g}$ be a Lie algebra. $X$ is an $(n+1)$-dimensional manifold, and we define $\mathcal{X}=T[1] X$. We consider $\mathcal{M}=T^{*}[n] \mathfrak{g}[1] \simeq \mathfrak{g}[1] \oplus \mathfrak{g}^{*}[n-1]$ with a point base manifold, $M=\{p t\}$. Let $\boldsymbol{q}^{a}$ be a section of $\left.T^{*}[1] X \otimes \boldsymbol{x}^{*}(\mathfrak{g}[1]]\right)$ of degree 1 , and $\boldsymbol{p}_{a}$ be a section of $\left.T^{*}[1] X \otimes \boldsymbol{x}^{*}\left(\mathfrak{g}^{*}[n-1]\right]\right)$ of degree $n$. Here, we denote $a(1)=a, b(1)=b, \cdots$. The P-structure is defined as

$$
\boldsymbol{\omega}=\int_{\mathcal{X}} d^{n+1} \sigma d^{n+1} \theta(-1)^{n|q|} \delta \boldsymbol{q}^{a} \wedge \delta \boldsymbol{p}_{a}
$$

The curvature is defined as $\boldsymbol{F}^{a}=\boldsymbol{d} \boldsymbol{q}^{a}+(-1)^{n} \frac{1}{2} f_{b c}^{a} \boldsymbol{q}^{b} \boldsymbol{q}^{c}$. The BV action is

$$
\begin{aligned}
S & =\int_{\mathcal{X}} d^{n+1} \sigma d^{n+1} \theta\left((-1)^{n} \boldsymbol{p}_{a} \boldsymbol{F}^{a}\right) \\
& =\int_{\mathcal{X}} d^{n+1} \sigma d^{n+1} \theta\left((-1)^{n} \boldsymbol{p}_{a} \boldsymbol{d} \boldsymbol{q}^{a}+\frac{1}{2} f^{a}{ }_{b c} \boldsymbol{p}_{a} \boldsymbol{q}^{b} \boldsymbol{q}^{c}\right)
\end{aligned}
$$

The master equation $\{S, S\}=0$ is easily confirmed. This action is equivalent to the BV formalism of a nonabelian BF theory in $n+1$ dimensions. [32, 41]

\subsubsection{Nonassociative Topological Field Theory}

We consider the QP-structure that was presented in Example 5.4.3. We obtain a TFT with a nontrivial nonassociativity based on a Lie n-algebroid structure.

$\mathcal{M}$ is a QP-manifold of degree $n, X$ is an $(n+1)$-dimensional manifold, and $\mathcal{X}=T[1] X$. From the Q-structure $\Theta$ in Example 5.4.3, the BV action $S=S_{0}+S_{1}$ on $\operatorname{Map}(\mathcal{X}, \mathcal{M})$ is 
constructed by the AKSZ construction. When $n$ is odd, $S_{0}$ has the form of equation (8.93), and when $n$ is even, it has the form of equation (8.94). $S_{1}$ has the following expression:

$$
S_{1}=\int_{\mathcal{X}} \mu \mathrm{ev}^{*} \Theta=\int_{\mathcal{X}} \mu \mathrm{ev}^{*}\left(\Theta_{0}+\Theta_{2}+\Theta_{3}+\cdots+\Theta_{n}\right),
$$

where the $\Theta_{i}$ 's are given in (5.79) and (5.80). After transgression, we obtain the superfield expressions,

$$
\int_{\mathcal{X}} \mu \mathrm{ev}^{*} \Theta_{0}=\int_{\mathcal{X}} d^{n+1} \sigma d^{n+1} \theta\left(f_{0}^{a(0)}{ }_{b(1)}(\boldsymbol{x}) \boldsymbol{\xi}_{a(0)} \boldsymbol{q}^{b(1)}\right)
$$

and

$$
\begin{aligned}
& \int_{\mathcal{X}} \mu \mathrm{ev}^{*} \Theta_{i} \\
= & \int_{\mathcal{X}} d^{n+1} \sigma d^{n+1} \theta\left(\frac{1}{i !} f_{i, a(n-i+1) b_{1}(1) \cdots b_{i}(1)}(\boldsymbol{x}) \boldsymbol{e}^{a(n-i+1)} \boldsymbol{q}^{b_{1}(1)} \cdots \boldsymbol{q}^{b_{i}(1)}\right) .
\end{aligned}
$$

In particular, for the $(n+1)$-form $\Theta_{n}$,

$$
\begin{aligned}
& \int_{\mathcal{X}} \mu \mathrm{ev}^{*} \Theta_{n} \\
= & \int_{\mathcal{X}} d^{n+1} \sigma d^{n+1} \theta\left(\frac{1}{(n+1) !} f_{n, b_{0}(1) b_{1}(1) \cdots b_{n}(1)}(\boldsymbol{x}) \boldsymbol{q}^{b_{0}(1)} \boldsymbol{q}^{b_{1}(1)} \cdots \boldsymbol{q}^{b_{n}(1)}\right) .
\end{aligned}
$$

The master equation $\{S, S\}=0$ defines the structure of the $(i+1)$-forms $\Theta_{i}$.

\section{AKSZ Sigma Models with Boundary}

So far, we have considered AKSZ sigma models on a closed base manifold $X$. In this section, we will consider AKSZ models, where the base manifold $X$ has boundaries. These have important applications. In the case where $n=1$, it corresponds to a topological open string and it yields the deformation quantization formulas [33]. The quantization of the $n=1$ case will be discussed below. If $n \geq 2$, the theory describes a topological open $n$-brane [116, 66].

\section{$10.1 n=2$ : WZ-Poisson Sigma Model}

We will explain the construction of the AKSZ theory with boundary using the WZ-Poisson sigma model, the simplest nontrivial example. Nontrivial boundary structures are described in supergeometry terminology. 
We take $n=2$ and the target graded manifold $\mathcal{M}=T^{*}[2] T^{*}[1] M$. As discussed, $T^{*}[2] T^{*}[1] M$ has a natural QP-manifold structure. Let $x^{i}$ be a coordinate of degree 0 on $M, q^{i}$ be a coordinate of degree 1 on the fiber of $T[1] M, p_{i}$ be a coordinate of degree 1 on the fiber of $T^{*}[1] M$, and $\xi_{i}$ be a coordinate of degree 2 on the fiber of $T^{*}[2] M$.

We take the following P-structure:

$$
\omega=\delta x^{i} \wedge \delta \xi_{i}+\delta q^{i} \wedge \delta p_{i}
$$

By introducing a 3-form $H$ on $M$, the Q-structure function is defined as

$$
\Theta=\xi_{i} q^{i}+\frac{1}{3 !} H_{i j k}(x) q^{i} q^{j} q^{k}
$$

Note that $\{\Theta, \Theta\}=0$ is equivalent to $d H=0$.

Let us consider a three-dimensional manifold $X$ with boundary $\partial X$. The AKSZ construction defines a topological sigma model on $\operatorname{Map}\left(T[1] X, T^{*}[2] T[1] M\right)$. This model is a special case of the Courant sigma model on an open manifold. The P-structure becomes

$$
\boldsymbol{\omega}=\int_{\mathcal{X}} d^{3} \sigma d^{3} \theta\left(\delta \boldsymbol{x}^{i} \wedge \delta \boldsymbol{\xi}_{i}+\delta \boldsymbol{p}_{i} \wedge \delta \boldsymbol{q}^{i}\right)
$$

The Q-structure BV action has the following form:

$$
S=\int_{\mathcal{X}} d^{3} \sigma d^{3} \theta\left(-\boldsymbol{\xi}_{i} \boldsymbol{d} \boldsymbol{x}^{i}+\boldsymbol{q}^{i} \boldsymbol{d} \boldsymbol{p}_{i}+\boldsymbol{\xi}_{i} \boldsymbol{q}^{i}+\frac{1}{3 !} H_{i j k}(\boldsymbol{x}) \boldsymbol{q}^{i} \boldsymbol{q}^{j} \boldsymbol{q}^{k}\right) .
$$

We need to determine the boundary conditions to complete the theory. Consistency with the variation principle restricts the possible boundary conditions. The variation $\delta S$ is

$$
\delta S=\int_{\mathcal{X}} d^{3} \sigma d^{3} \theta\left(-\delta \boldsymbol{\xi}_{i} \boldsymbol{d} \boldsymbol{x}^{i}-\boldsymbol{\xi}_{i} \boldsymbol{d} \delta \boldsymbol{x}^{i}+\delta \boldsymbol{q}^{i} \boldsymbol{d} \boldsymbol{p}_{i}+\boldsymbol{q}^{i} \boldsymbol{d} \delta \boldsymbol{p}_{i}+\cdots\right)
$$

To derive the equations of motion, we use integration by parts for the terms $-\boldsymbol{\xi}_{i} \boldsymbol{d} \delta \boldsymbol{x}^{i}+\boldsymbol{q}^{i} \boldsymbol{d} \delta \boldsymbol{p}_{i}$. The boundary terms must vanish, i.e.,

$$
\left.\delta S\right|_{\partial \mathcal{X}}=\int_{\partial \mathcal{X}} d^{2} \sigma d^{2} \theta\left(-\boldsymbol{\xi}_{i} \delta \boldsymbol{x}^{i}-\boldsymbol{q}^{i} \delta \boldsymbol{p}_{i}\right)=0
$$

Any boundary condition must be consistent with equation (10.108).

Two kinds of local boundary conditions are possible: $\boldsymbol{\xi}_{/ / i}=0$ or $\delta \boldsymbol{x}_{/ /}^{i}=0$, and $\boldsymbol{q}_{/ /}^{i}=0$ or $\delta \boldsymbol{p}_{/ / i}=0$, where $/ /$ indicates the component that is parallel to the boundary ${ }^{r}$ As an example, ${ }^{r}$ Hybrids of these boundary conditions are also possible. 
we take the boundary conditions $\boldsymbol{\xi}_{/ / i}=0$ and $\boldsymbol{q}_{/ /}^{i}=0$ on $\partial \mathcal{X}$. These boundary conditions can be written using the components of the superfields as follows: $\xi_{i}^{(0)}=\xi_{0 i}^{(1)}=\xi_{1 i}^{(1)}=\xi_{01 i}^{(2)}=0$ and $q^{(0) i}=q_{0}^{(1) i}=q_{1}^{(1) i}=q_{01}^{(2) i}=0$ on $\partial \mathcal{X}$.

Another consistency condition is that the boundary conditions must not break the classical master equation $\{S, S\}=0$. Direct computation using the BV action (10.107) gives

$$
\{S, S\}=\int_{\partial \mathcal{X}} d^{2} \sigma d^{2} \theta\left(-\boldsymbol{\xi}_{i} \boldsymbol{d} \boldsymbol{x}^{i}+\boldsymbol{q}^{i} \boldsymbol{d} \boldsymbol{p}_{i}+\boldsymbol{\xi}_{i} \boldsymbol{q}^{i}+\frac{1}{3 !} H_{i j k}(\boldsymbol{x}) \boldsymbol{q}^{i} \boldsymbol{q}^{j} \boldsymbol{q}^{k}\right) .
$$

The boundary conditions $\boldsymbol{\xi}_{/ / i}=0$ and $\boldsymbol{q}_{/ /}^{i}=0$ are consistent with the classical master equation. The kinetic terms on the right-hand side in equation (10.109) vanish on the boundary:

$$
\int_{\partial \mathcal{X}} d^{2} \sigma d^{2} \theta \widehat{\vartheta}=\int_{\partial \mathcal{X}} d^{2} \sigma d^{2} \theta\left(-\boldsymbol{\xi}_{i} \boldsymbol{d} \boldsymbol{x}^{i}+\boldsymbol{q}^{i} \boldsymbol{d} \boldsymbol{p}_{i}\right)=0 .
$$

The interaction terms in equation (10.109) also vanish:

$$
\int_{\partial \mathcal{X}} d^{2} \sigma d^{2} \theta \widehat{\Theta}=\int_{\partial \mathcal{X}} d^{2} \sigma d^{2} \theta\left(\boldsymbol{\xi}_{i} \boldsymbol{q}^{i}+\frac{1}{3 !} H_{i j k}(\boldsymbol{x}) \boldsymbol{q}^{i} \boldsymbol{q}^{j} \boldsymbol{q}^{k}\right)=0 .
$$

It is accidental that the second condition does not impose a new condition. Generally, we have more conditions on the boundary, such as in the next example.

The consistency of the boundary conditions is described in the language of the target QP-manifold $\mathcal{M}$. Equation (10.110) is satisfied if $\xi_{i}=q^{i}=0$. From equation (10.104), this is satisfied if the image of a boundary is in a Lagrangian subspace of the P-structure $\omega$. Equation (10.111) is satisfied if $\left.\widehat{\Theta}\right|_{\partial \mathcal{X}}=0$, that is, the Q-structure vanishes $(\Theta=0)$ on the Lagrangian subspace.

Note that there exists an ambiguity in the total derivatives of $S_{0}$, and this comes from the ambiguity in the expression for the local coordinates of $\vartheta$. Here, we choose an $S_{0}$ such that the classical master equation is satisfied if we take $\left.\widehat{\Theta}\right|_{\partial \mathcal{X}}=0$. For example, if we use the boundary condition $\xi_{i}=p_{i}=0$, then we should take $S_{0}=\int_{\mathcal{X}} d^{3} \sigma d^{3} \theta\left(-\boldsymbol{\xi}_{i} \boldsymbol{d} \boldsymbol{x}^{i}+\boldsymbol{p}_{i} \boldsymbol{d} \boldsymbol{q}^{i}\right)$.

We can change the boundary condition by introducing consistent boundary terms. For the present example, the boundary terms must be pullbacks of a degree two function $\alpha$ by the transgression map, $\mu_{*} \mathrm{ev}^{*} \alpha$. As an example, we take $\alpha=\frac{1}{2} f^{i j}(x) p_{i} p_{j}$ [116] and find consistency 
conditions for $H_{i j k}(x)$ and $f^{i j}(x)$ s The modified action is given by

$$
\begin{aligned}
S= & \int_{\mathcal{X}} d^{3} \sigma d^{3} \theta\left(-\boldsymbol{\xi}_{i} \boldsymbol{d} \boldsymbol{x}^{i}+\boldsymbol{q}^{i} \boldsymbol{d} \boldsymbol{p}_{i}+\boldsymbol{\xi}_{i} \boldsymbol{q}^{i}+\frac{1}{3 !} H_{i j k}(\boldsymbol{x}) \boldsymbol{q}^{i} \boldsymbol{q}^{j} \boldsymbol{q}^{k}\right) \\
& -\int_{\partial \mathcal{X}} d^{2} \sigma d^{2} \theta \frac{1}{2} f^{i j}(\boldsymbol{x}) \boldsymbol{p}_{i} \boldsymbol{p}_{j} .
\end{aligned}
$$

In order to derive the equations of motion from the variation of $\delta S$, the following boundary integral must vanish:

$$
\left.\delta S\right|_{\partial \mathcal{X}}=\int_{\partial \mathcal{X}} d^{2} \sigma d^{2} \theta\left[\left(-\boldsymbol{\xi}_{i}-\frac{1}{2} \frac{\partial f^{j k}(\boldsymbol{x})}{\partial \boldsymbol{x}^{i}} \boldsymbol{p}_{j} \boldsymbol{p}_{k}\right) \delta \boldsymbol{x}^{i}+\left(-\boldsymbol{q}^{i}+f^{i j}(\boldsymbol{x}) \boldsymbol{p}_{j}\right) \delta \boldsymbol{p}_{i}\right] .
$$

This determines the boundary conditions as

$$
\left.\boldsymbol{\xi}_{i}\right|_{/ /}=-\left.\left.\frac{1}{2} \frac{\partial f^{j k}}{\partial \boldsymbol{x}^{i}}(\boldsymbol{x}) \boldsymbol{p}_{j} \boldsymbol{p}_{k}\right|_{/ /,} \quad \boldsymbol{q}^{i}\right|_{/ /}=\left.f^{i j}(\boldsymbol{x}) \boldsymbol{p}_{j}\right|_{/ /}
$$

In addition, we must also consider a boundary term in $\{S, S\}$. In this example, the classical master equation, $\{S, S\}=0$, requires the integrand of $S_{1}$ to be zero on the boundary $t^{t}$

$$
\left.\left(\boldsymbol{\xi}_{i} \boldsymbol{q}^{i}+\frac{1}{3 !} H_{i j k}(\boldsymbol{x}) \boldsymbol{q}^{i} \boldsymbol{q}^{j} \boldsymbol{q}^{k}\right)\right|_{/ /}=0 .
$$

Equations (10.113) and (10.114) show that the image of the boundary must satisfy the following conditions,

$$
\begin{aligned}
& \xi_{i} q^{i}+\frac{1}{3 !} H_{i j k}(x) q^{i} q^{j} q^{k}=0, \\
& \xi_{i}=-\frac{1}{2} \frac{\partial f^{j k}}{\partial x^{i}}(x) p_{j} p_{k}, \\
& q^{i}=f^{i j}(x) p_{j} .
\end{aligned}
$$

This means that equation (10.115) is satisfied on the Lagrangian subspace $\mathcal{L}_{\alpha}$ of a target QP-manifold $\mathcal{M}$ defined by (10.116) and (10.117). By substituting equations (10.116) and ${ }^{s}$ Equation (10.112) is just one example of a boundary term; we can consider more general boundary terms, such as

$$
-\int_{\partial \mathcal{X}} d^{2} \sigma d^{2} \theta\left(\boldsymbol{p}_{i} \boldsymbol{d} \boldsymbol{x}^{i}+\frac{1}{2} f^{i j}(\boldsymbol{x}) \boldsymbol{p}_{i} \boldsymbol{p}_{j}+g^{i}{ }_{j}(\boldsymbol{x}) \boldsymbol{p}_{i} \boldsymbol{q}^{j}+\frac{1}{2} h_{i j}(\boldsymbol{x}) \boldsymbol{q}^{i} \boldsymbol{q}^{j}\right) .
$$

${ }^{t}$ Equation (10.114) is the same as equation (10.111). We can prove that this condition does not depend on the boundary conditions. 
(10.117) into equation (10.115), we obtain the geometric structures on the image of the boundary $\partial X$

$$
\begin{aligned}
& \xi_{i} q^{i}+\frac{1}{3 !} H_{i j k}(x) q^{i} q^{j} q^{k} \\
= & -\frac{1}{2} \frac{\partial f^{j k}}{\partial x^{l}}(x) f^{l i}(x) p_{j} p_{k} p_{i}+\frac{1}{3 !} H_{i j k}(x) f^{i l}(x) f^{j m}(x) f^{k n}(x) p_{l} p_{m} p_{n} \\
= & 0
\end{aligned}
$$

If we define a bivector field $\pi=\frac{1}{2} f^{i j}(x) \partial_{i} \wedge \partial_{j}$, then equation (10.118) is equivalent to

$$
[\pi, \pi]_{S}=\wedge^{3} \pi^{\#} H
$$

Here, $[-,-]_{S}$ is the Schouten-Nijenhuis bracket on the space of multivector fields $\Gamma\left(\wedge^{\bullet} T M\right)$, which is an odd Lie bracket on the exterior algebra such that $\partial_{i} \wedge \partial_{j}=-\partial_{j} \wedge \partial_{i}$. The operation $\pi^{\#}: T^{*} M \rightarrow T M$ is locally defined by $\frac{1}{2} f^{i j}(x) \partial_{i} \wedge \partial_{j}\left(d x^{k}\right)=f^{k j}(x) \partial_{j}$. Equation (10.119) is called a twisted Poisson structure [137].

The ghost number 0 part of the BV action, equation (10.112), becomes

$$
\begin{aligned}
\left.S\right|_{0}= & \int_{X}\left(-\xi_{i}^{(2)} \wedge d x^{i}+q^{(1) i} \wedge d p_{i}^{(1)}+\xi_{i}^{(2)} \wedge q^{(1) i}+\frac{1}{3 !} H_{i j k}(x) q^{(1) i} \wedge q^{(1) j} \wedge q^{(1) k}\right) \\
& -\int_{\partial X} \frac{1}{2} f^{i j}(x) p_{i}^{(1)} \wedge p_{j}^{(1)}
\end{aligned}
$$

after integration with respect to $\theta^{\mu}$, where $x=x^{(0)}$. Integrating out $\xi_{i}^{(2)}$, we obtain a topological field theory in two dimensions with a Wess-Zumino term:

$$
\left.S\right|_{0}=\int_{\partial X}\left(-p_{i}^{(1)} \wedge d x^{i}-\frac{1}{2} f^{i j}(x) p_{i}^{(1)} \wedge p_{j}^{(1)}\right)+\int_{X} \frac{1}{3 !} H_{i j k}(x) d x^{i} \wedge d x^{j} \wedge d x^{k} .
$$

This model is called the WZ-Poisson sigma model or the twisted Poisson sigma model [91]. The constraints are first class if and only if the target space manifold has a twisted Poisson structure.

\subsection{General Structures of AKSZ Sigma Models with Boundary}

In the previous subsection, a typical example for boundary structures of AKSZ sigma models was presented. In this subsection, we discuss the general theory in $n+1$ dimensions.

Assume that $X$ is an $(n+1)$-dimensional manifold with boundary, $\partial X \neq \emptyset$. Let $\mathcal{M}$ be a QP-manifold of degree $n$. Then, by the AKSZ construction, a topological sigma model on 
$\operatorname{Map}(T[1] X, \mathcal{M})$ can be constructed. The boundary conditions on $\partial X$ must be consistent with the QP-structure.

First, let us take a Q-structure function $S=S_{0}+S_{1}=\iota_{\hat{D}} \mu_{*} \mathrm{ev}^{*} \vartheta+\mu_{*} \mathrm{ev}^{*} \Theta$ without boundary terms. Then, $\{S, S\}$ yields the integrated boundary terms,

$$
\{S, S\}=\iota_{\hat{D}} \mu_{\partial \mathcal{X}_{*}}\left(i_{\partial} \times \mathrm{id}\right)^{*} \mathrm{ev}^{*} \vartheta+\mu_{\partial \mathcal{X}_{*}}\left(i_{\partial} \times \mathrm{id}\right)^{*} \mathrm{ev}^{*} \Theta,
$$

where $\mu_{\partial \mathcal{X}}$ is the boundary measure induced from $\mu$ on $\partial \mathcal{X}$ by the inclusion map $i_{\partial}: \partial \mathcal{X} \longrightarrow$ $\mathcal{X}$. The map $\left(i_{\partial} \times \text { id }\right)^{*}: \Omega^{\bullet}(\mathcal{X} \times \mathcal{M}) \longrightarrow \Omega^{\bullet}(\partial \mathcal{X} \times \mathcal{M})$ is the restriction of the bulk graded differential forms on the mapping space to the boundary $\partial \mathcal{X}$. In order to satisfy the master equation, the right-hand side of equation (10.121) must vanish. Thus we obtain the following theorem,

Theorem 10.1 Assume that $\partial \mathcal{X} \neq \emptyset .\{S, S\}=0$ requires $\iota_{\hat{D}} \mu_{\partial \mathcal{X}_{*}}\left(i_{\partial} \times \mathrm{id}\right)^{*} \operatorname{ev}^{*} \vartheta+\mu_{\partial \mathcal{X}_{*}}\left(i_{\partial} \times\right.$ id) ${ }^{*} \mathrm{ev}^{*} \Theta=0$.

If we consider the consistency with the variational principle of a field theory, the two terms must vanish independently. We explain this using the local coordinate expression.

The kinetic term in the AKSZ sigma model is

$$
S_{0}=\int_{\mathcal{X}} d^{n+1} \sigma d^{n+1} \theta \sum_{0 \leq i \leq\lfloor n / 2\rfloor}(-1)^{n+1-i} \boldsymbol{p}_{a(i)} \boldsymbol{d} \boldsymbol{q}^{a(i)} .
$$

In order to derive the equations of motion, we take the variation. We find that the boundary integration of the variation of the total action, should vanish for consistency:

$$
\left.\delta S\right|_{\partial \mathcal{X}}=\int_{\partial \mathcal{X}} d^{n} \sigma d^{n} \theta \sum_{0 \leq i \leq\lfloor n / 2\rfloor}(-1)^{n+1-i} \boldsymbol{p}_{a(i)} \delta \boldsymbol{q}^{a(i)}=0 .
$$

This imposes the boundary conditions $\boldsymbol{p}_{a(i)}=0$ or $\delta \boldsymbol{q}^{a(i)}=0$ on $\partial X$. This implies that the image of the boundary lies in a Lagrangian submanifold $\mathcal{L} \subset \mathcal{M}$, which is the zero locus of $\vartheta,\left.\vartheta\right|_{\mathcal{L}}=0$, on the target space. Under this condition, the first term in equation (10.121), $\iota_{\hat{D}} \mu_{\partial \mathcal{X}}\left(i_{\partial} \times \mathrm{id}\right)^{*} \mathrm{ev}^{*} \vartheta$, vanishes. Therefore, Theorem 10.1 reduces to a simpler form, that is, the condition that the second term vanishes. This can be reinterpreted as a condition on $\Theta$ on the target space.

Proposition 10.2 Let $\mathcal{L}$ be a Lagrangian submanifold of $\mathcal{M}$, i.e., $\left.\vartheta\right|_{\mathcal{L}}=0$. Then $\{S, S\}=0$ is satisfied if $\left.\Theta\right|_{\mathcal{L}}=0$. [66] 


\subsection{Canonical Transformation of Q-structure Function}

In the remainder of this section, we discuss the general theory of boundary terms. Let us define an exponential adjoint operation $e^{\delta_{\alpha}}$ on a general QP-manifold $\mathcal{M}$,

$$
e^{\delta_{\alpha}} \Theta=\Theta+\{\Theta, \alpha\}+\frac{1}{2}\{\{\Theta, \alpha\}, \alpha\}+\cdots
$$

where $\alpha \in C^{\infty}(\mathcal{M})$.

Definition 10.3 Let $(\mathcal{M}, \omega, \Theta)$ be a QP-manifold of degree $n, \alpha \in C^{\infty}(\mathcal{M})$ be a function of degree $n$, then, $e^{\delta_{\alpha}}$ is called a twist by $\alpha$.

This transformation preserves degree, since $\alpha$ is of degree $n$. Note that a twist satisfies $\left\{e^{\delta_{\alpha}} f, e^{\delta_{\alpha}} g\right\}=e^{\delta_{\alpha}}\{f, g\}$ for any function $f, g \in C^{\infty}(\mathcal{M})$, therefore, the twist by $\alpha$ is a canonical transformation.

Now we consider a canonical transformation of a QP-manifold $(\mathcal{M}, \omega, \Theta)$ by a twist $e^{\delta_{\alpha}}$. Since the Q-structure function $\Theta$ changes to $e^{\delta_{\alpha}} \Theta$, the Q-structure function in the corresponding AKSZ sigma model is changed to

$$
\begin{aligned}
S & =S_{0}+S_{1} \\
& =\iota_{\hat{D}} \mu_{*} \mathrm{ev}^{*} \vartheta+\mu_{*} \mathrm{ev}^{*} e^{\delta_{\alpha}} \Theta .
\end{aligned}
$$

If $\partial X=\emptyset$, the consistency condition of the theory is not changed, since a canonical transformation preserves the graded Poisson bracket and the classical master equation. However, if $\partial X \neq \emptyset$, the twist changes the boundary conditions. Applying Proposition 10.1 to equation (10.125), we obtain the following conditions on $\alpha$ for the consistent boundary conditions of the AKSZ sigma models.

Proposition 10.4 Assume $\partial \mathcal{X} \neq \emptyset$. Let $(\mathcal{M}, \omega, \Theta)$ be a $Q P$-manifold of degree $n$, $\mathcal{L}$ be a Lagrangian submanifold of $\mathcal{M}$, which is the zero locus of $\vartheta$, and $\alpha \in C^{\infty}(\mathcal{M})$ be a function of degree $n$. If the twist generated by $\alpha$ vanishes on $\mathcal{L},\left.e^{\delta_{\alpha}} \Theta\right|_{\mathcal{L}}=0$, then the Q-structure function (10.125) satisfies the classical master equation $\{S, S\}=0$. [66]

A function $\alpha$ with the property defined in Proposition 10.4 is called a Poisson function [141, 97] or a canonical function [82]. The structures for general $n$ have been analyzed in Ref. [82]. 


\subsection{From Twist to Boundary Terms}

In this subsection, we show that a canonical function $\alpha$, defined in the previous section, generates a boundary term. Let $I=\mu_{*} \mathrm{ev}^{*} \alpha$ be a functional constructed by a transgression of $\alpha$. In equation (10.125), the change in the Q-structure by the twist is converted into the change in the P-structure by the following inverse canonical transformation on the mapping space,

$$
\begin{aligned}
S^{\prime} & =e^{-\delta_{I}} S \\
& =e^{-\delta_{I}} S_{0}+\mu_{*} \mathrm{ev}^{*} e^{-\delta_{\alpha}} e^{\delta_{\alpha}} \Theta \\
& =e^{-\delta_{I}} S_{0}+\mu_{*} \mathrm{ev}^{*} \Theta .
\end{aligned}
$$

This QP-structure $\left(\boldsymbol{\omega}^{\prime}=-d\left(e^{-\delta_{I}} S_{0}\right), S^{\prime}\right)$ is equivalent to the original QP-structure $(\boldsymbol{\omega}, S)$. 66]

For a physical interpretation of $\alpha$, we consider the simple special case in which $\alpha$ satisfies $\{\alpha, \alpha\}=0$, and thus $\{I, I\}=0$. Then, since $e^{-\delta_{I}} S_{0}=S_{0}-\left\{S_{0}, I\right\}$, the BV action becomes

$$
S^{\prime}=S_{0}-\left\{S_{0}, I\right\}+\mu_{*} \mathrm{ev}^{*} \Theta
$$

The second term, $-\left\{S_{0}, I\right\}$, is nothing but a boundary term:

$$
\begin{aligned}
-\left\{S_{0}, I\right\} & =-\left\{S_{0}, \int_{\mathcal{X}} \mu \mathrm{ev}^{*} \alpha\right\} \\
& =\int_{\mathcal{X}} d^{n+1} \sigma d^{n+1} \theta \boldsymbol{d e v}^{*} \alpha=\int_{\partial \mathcal{X}} d^{n+1} \sigma d^{n+1} \theta \mathrm{ev}^{*} \alpha .
\end{aligned}
$$

Therefore, a canonical transformation by a twist induces a boundary term generated by the $\alpha$ in the BV action $S$. The boundary term generally carries a nonzero charge. In physics, this charge can be identified with the number of $n$-branes, and the above action (10.127) defines a so-called topological open $n$-brane theory. This structure has been applied to the analysis of T-duality geometry. [14] If $\{\alpha, \alpha\} \neq 0$, we cannot make a simple interpretation as local boundary terms, but it still gives a consistent deformation of an AKSZ sigma model. As a special case of this construction, the Nambu-Poisson structures are realized by the AKSZ sigma models on a manifold with boundary. [25]

In this section, we have discussed Dirichlet-like fixed boundary conditions. We can also impose Neumann-like free boundary conditions. The AKSZ sigma models with free boundary 
conditions are called the AKSZ-BFV theories on a manifold with boundary, and they have been analyzed in Ref. [38, 39].

\section{Topological Strings from AKSZ Sigma Models}

In this section, we discuss derivations of the A- and B-models [151] from the AKSZ sigma models in two dimensions, which is equivalent to the Poisson sigma model. The A- and B-models are derived by gauge fixing of this AKSZ sigma model. [5]

\section{$11.1 \quad$ A-Model}

Let the worldsheet $X=\Sigma$ be a compact Riemann surface and the target space $M$ be a Kähler manifold. Let us consider the AKSZ formalism of the Poisson sigma model in Example 9.1.1. Here, we take the theory where $S_{0}=0$ in the Q-structure BV action (9.99), i.e.,

$$
S=S_{1}=\int_{T[1] \Sigma} d^{2} \sigma d^{2} \theta f^{i j}(\boldsymbol{x}) \boldsymbol{\xi}_{i} \boldsymbol{\xi}_{j}
$$

Here, we take the normalization of $S_{1}$ in Ref. [5]. The classical master equation, $\left\{S_{1}, S_{1}\right\}=0$, is satisfied if $f^{i j}(x)$ satisfies equation (5.41) as in the case of the Poisson sigma model, i.e., if $M$ is a Poisson manifold. This condition is satisfied on a Kähler manifold $M$, by taking $f^{i j}$ as the inverse of the Kähler form. As in Example 9.1.1, the superfields $\left(\boldsymbol{x}^{i}, \boldsymbol{\xi}_{i}\right)$ of degree $(0,1)$ can be identified with $\left(\phi^{i}, \boldsymbol{A}_{i}\right)$ in Section 2.4. The superfields are expanded in the supercoordinate $\theta^{\mu}$,

$$
\begin{aligned}
& \boldsymbol{x}^{i}=\boldsymbol{\phi}^{i}=\phi^{i}+A^{+i}+c^{+i}\left(=x^{(0) i}+x^{(1) i}+x^{(2) i}\right) \\
& \boldsymbol{\xi}_{i}=\boldsymbol{A}_{i}=-c_{i}+A_{i}+\phi_{i}^{+}\left(=\xi_{i}^{(0)}+\xi_{i}^{(1)}+\xi_{i}^{(2)}\right)
\end{aligned}
$$

We take the complex coordinates $(z, \bar{z})$ on the worldsheet $\Sigma$ and on the target space $M$ with holomorphic and antiholomorphic indices $i=(a, \dot{a})$. Let $J_{j}^{i}$ be a complex structure and $g_{i j}$ be a Kähler metric. Then, the inverse of the Kähler form $f^{i j}$ is expressed as $f^{i j}=-J^{i}{ }_{k} g^{k j}$. We decompose the holomorphic and antiholomorphic parts of the fields with respect to the worldsheet complex structure. $A_{z}^{+i}=-A_{0}^{+i}+i A_{1}^{+i}$ and $A_{\bar{z}}^{+i}=A_{0}^{+i}+i A_{1}^{+i}, A_{z i}=-A_{1 i}-i A_{0 i}$ 
and $A_{\bar{z} i}=A_{1 i}-i A_{0 i}, \phi_{z \bar{z} i}^{+}=2 i \phi_{i}^{*}$ and $c_{z \bar{z}}{ }^{+i}=2 i c^{* i}$. The BV antibrackets are

$$
\begin{aligned}
& \left\{A_{z i}, A_{\bar{z}^{\prime}}^{+j}\right\}=2 \delta_{i}^{j} \delta\left(z-z^{\prime}\right) \delta\left(\bar{z}-\bar{z}^{\prime}\right), \quad\left\{A_{\bar{z} i}, A_{z^{\prime}}^{+j}\right\}=2 \delta_{i}^{j} \delta\left(z-z^{\prime}\right) \delta\left(\bar{z}-\bar{z}^{\prime}\right), \\
& \left\{\phi^{i}, \phi_{z \bar{z}}{ }^{+}\right\}=2 i \delta^{i}{ }_{j} \delta\left(z-z^{\prime}\right) \delta\left(\bar{z}-\bar{z}^{\prime}\right), \quad\left\{c_{i}, c_{z \bar{z}}{ }^{+j}\right\}=2 i \delta_{i}{ }^{j} \delta\left(z-z^{\prime}\right) \delta\left(\bar{z}-\bar{z}^{\prime}\right),
\end{aligned}
$$

and all other antibrackets are zero. Taking linear combinations of the fields, we obtain the complex fields with respect to the target complex structure. For example, for $A_{z i}, A_{\bar{z} i}, A_{z}^{+i}$ and $A_{\bar{z}}^{+i}$, we take linear combinations such that $\overline{A_{z}^{+a}}=A_{\bar{z}}^{+\dot{a}}, \overline{A_{z a}}=A_{\bar{z} \dot{a}}$. Their BV brackets are

$$
\left\{A_{z a}, A_{\bar{z}^{\prime}}^{+b}\right\}=\delta_{a}^{b} \delta\left(z-z^{\prime}\right) \delta\left(\bar{z}-\bar{z}^{\prime}\right), \quad\left\{A_{\bar{z} a}, A_{z^{\prime}}^{+b}\right\}=\delta_{a}^{b} \delta\left(z-z^{\prime}\right) \delta\left(\bar{z}-\bar{z}^{\prime}\right)
$$

and their complex conjugates.

If the gauge symmetry of the theory is partially fixed by the BV gauge fixing procedure, the action reduces to the A-model action given in Ref. [151]. We fix $c^{+i}, A_{\bar{z} a}, A_{z \dot{a}}$ and $\phi_{i}^{+}$by taking the following gauge fixing fermion

$$
\Psi=\int_{T[1] \Sigma} d^{2} z g_{a \dot{a}}(\phi)\left(A_{z}^{+\dot{a}} \partial_{\bar{z}} \phi^{a}-A_{\bar{z}}^{+a} \partial_{z} \phi^{\dot{a}}\right) .
$$

We obtain the gauge fixing conditions,

$$
\begin{aligned}
& c^{+i}=0, \\
& A_{\dot{a}}^{z}=i g_{a \dot{a}}(\phi) \partial_{\bar{z}} \phi^{a}, \\
& A^{\bar{z}}{ }_{a}=-i g_{a \dot{a}}(\phi) \partial_{z} \phi^{\dot{a}}, \\
& \phi_{z \bar{z}}^{+}=-i \partial_{\bar{z}}\left(g_{a \dot{a}}(\phi) A_{z}^{+\dot{a}}\right), \\
& \phi_{z \bar{z}_{\dot{a}}}=i \partial_{z}\left(g_{a \dot{a}}(\phi) A_{\bar{z}}^{+a}\right) .
\end{aligned}
$$

Substituting equations (11.129) into equation (11.128) and integrating out $A_{z a}$ and $A_{\bar{z} \dot{a}}$, we obtain the original A-model action,

$$
S_{1}=\int_{\Sigma} d^{2} z\left(g_{a \dot{a}} \partial_{\bar{z}} \phi^{a} \partial_{z} \phi^{\dot{a}}-i \psi_{\bar{z}}^{a} D_{z} \chi_{a}-i \psi_{z}^{\dot{a}} D_{\bar{z}} \chi_{\dot{a}}+R_{a \dot{a}}^{b \dot{b}} \psi_{\bar{z}}^{a} \psi_{z}^{\dot{a}} \chi_{b} \chi_{\dot{b}}\right)
$$

where

$$
\chi_{i}=\frac{1}{2 i} c_{i}, \quad \psi_{\mu}^{a}=A_{\mu}^{+a}, \quad \psi_{\mu}^{\dot{a}}=A_{\mu}^{+\dot{a}}
$$


and

$$
\begin{aligned}
D_{z} \chi_{i} & =\partial_{z} \chi_{i}-\Gamma_{i j}^{k} \partial_{z} \phi^{j} \chi_{k}, \\
D_{\bar{z}} \chi_{i} & =\partial_{\bar{z}} \chi_{i}-\Gamma_{i j}^{k} \partial_{\bar{z}} \phi^{j} \chi_{k}
\end{aligned}
$$

and $\Gamma_{i j}^{k}$ is the Christoffel symbol on the target space.

\subsection{B-Model}

We start from Example 9.1.2, the QP-manifold realization of a complex structure on a smooth manifold $M$, and take local coordinates on the target space such that $J^{i}{ }_{j}=\left(\begin{array}{cc}0 & 1 \\ -1 & 0\end{array}\right)=$ $\epsilon^{i k} \delta_{k j}$. Then, the BV action (9.100) is simplified to

$$
S_{B}=\int_{\mathcal{X}} d^{2} z d^{2} \theta\left(\boldsymbol{\xi}_{i} \boldsymbol{d} \boldsymbol{x}^{i}-\boldsymbol{p}_{i} \boldsymbol{d} \boldsymbol{q}^{i}+\epsilon_{j}^{i} \boldsymbol{\xi}_{i} \boldsymbol{q}^{j}\right)
$$

The superfields can be expanded in $\theta^{\mu}$ as

$$
\begin{aligned}
& \boldsymbol{x}^{i}=x^{(0) i}+x^{(1) i}+x^{(2) i}, \\
& \boldsymbol{\xi}_{i}=\xi_{i}^{(0)}+\xi_{i}^{(1)}+\xi_{i}^{(2)}, \\
& \boldsymbol{q}^{i}=q^{(0) i}+q^{(1) i}+q^{(2) i}, \\
& \boldsymbol{p}_{i}=p_{i}^{(0)}+p_{i}^{(1)}+p_{i}^{(2)} .
\end{aligned}
$$

We consider partial gauge fixing, as in the A-model. Different gauge fixing conditions for the holomorphic and antiholomorphic parts are imposed as follows,

$$
\begin{aligned}
& x^{(1) \dot{a}}=0 \\
& x_{z \bar{z}}^{(2) a}+\Gamma_{b c}^{a} x_{z}^{(1) b} x_{\bar{z}}^{(1) c}=0 \\
& \xi_{\dot{a}}^{(0)}=0 \\
& \xi_{z a}^{(1)}+\Gamma_{a c}^{b} \xi_{b}^{(0)} x_{z}^{(1) c}=g_{a \dot{a}}(\phi) \partial_{z} x^{(0) \dot{a}} \\
& \xi_{\bar{z} a}^{(1)}-\Gamma_{a c}^{b} \xi_{b}^{(0)} x_{\bar{z}}^{(1) c}=g_{a \dot{a}}(\phi) \partial_{\bar{z}} x^{(0) \dot{a}}, \\
& \xi_{z \bar{z} a}^{(2)}=0, \quad \xi_{z \bar{z} \dot{a}}^{(2)}-R_{a \dot{a} b}^{c} x_{\bar{z}}^{(1) a} x_{z}^{(1) b} \xi_{c}^{(0)}=-\left(D_{z} x_{\bar{z}}^{(1) a}+D_{\bar{z}} x_{z}^{(1) a}\right) g_{a \dot{a}}, \\
& q^{(0) \dot{a}}=q^{(1) \dot{a}}=q^{(2) \dot{a}}=0 \\
& p_{a}^{(0)}=p_{a}^{(1)}=p_{a}^{(2)}=0 .
\end{aligned}
$$


Substituting equations (11.131) into equation (11.130), we obtain the original B-model action,

$$
\begin{aligned}
S= & \int_{\Sigma} d^{2} z\left(g_{i j} \partial_{z} \phi^{i} \partial_{\bar{z}} \phi^{j}+i \eta_{z}^{\dot{a}}\left(D_{z} \rho_{\bar{z}}^{a}+D_{\bar{z}} \rho_{z}^{a}\right) g_{a \dot{a}}+i \theta_{a}\left(D_{\bar{z}} \rho_{z}^{a}-D_{z} \rho_{\bar{z}}^{a}\right)\right. \\
& \left.-R_{a \dot{a} b \dot{b}} \rho_{z}^{a} \rho_{\bar{z}}^{b} \eta^{\dot{a}} \theta_{c} g^{c \dot{b}}\right)
\end{aligned}
$$

where $\phi^{i}=x^{(0) i}, \rho^{a}=x^{(1) a}, \theta_{a}=\xi_{a}^{(0)}$ and $\eta^{\dot{a}}=g^{a \dot{a}} p_{a}^{(0)}$.

\section{Quantization}

We discuss the quantization of the AKSZ sigma models in two dimensions as an important example. The quantization is carried out by the usual procedure of the BV formalism. Quantization in general dimensions is not well understood, yet.

\subsection{Poisson Sigma Model on a Disc}

The path integral quantization of the Poisson sigma model on a disc yields the Kontsevich deformation quantization formula on a Poisson manifold. [33] We briefly explain this model as an example of the quantization of an AKSZ sigma model. For details, we refer to Ref. 33.

\subsubsection{Deformation Quantization}

Recall that a Poisson manifold is a manifold $M$ with a Poisson bracket $\{-,-\}_{P B}$.

Definition 12.1 [deformation quantization] Let $M$ be a Poisson manifold and $C^{\infty}(M)[[\hbar]]$ be a set of formal power series on $C^{\infty}(M)$, where $\hbar$ is a formal parameter. A deformation quantization is a product (star product) * on $C^{\infty}(M)[[\hbar]]$ satisfying the following conditions:

(1) For $F, G \in C^{\infty}(M)[[\hbar]], F * G=\sum_{k}\left(\frac{i \hbar}{2}\right)^{k} \mathcal{B}_{k}(F, G)$ is bilinear, where $\mathcal{B}_{k}$ is a bidifferential operator such that $\mathcal{B}_{0}$ is a product, $\mathcal{B}_{0}(F, G)=F G$, and $\mathcal{B}_{1}$ is a Poisson bracket, $\mathcal{B}_{1}(F, G)=\{F, G\}_{P B}$.

(2) For $F, G, H \in C^{\infty}(M)[[\hbar]]$, * is associative, i.e.,

$$
(F * G) * H=F *(G * H)
$$


(3) Two star products $*$ and $*^{\prime}$ corresponding to the same Poisson bracket are equivalent if they coincide by the following linear transformation: $F^{\prime}=R F=\sum_{k}\left(\frac{i \hbar}{2}\right)^{k} \mathcal{D}_{k}(F)$, where $\mathcal{D}_{k}$ is a differential operator. i.e.

$$
F *^{\prime} G(x)=R^{-1}(R F * R G)
$$

We review the following theorem proved in Ref. [33].

Theorem 12.2 The correlation functions of the Poisson sigma model of observables on the boundary of a disc coincide with the star product formula on a Poisson manifold, called the Kontsevich formula. i.e.

$$
F * G(x)=\langle F(\phi(1)) G(\phi(0))\rangle=\int_{\phi(\infty)=x} \mathcal{D} \Phi F(\phi(1)) G(\phi(0)) e^{\frac{i}{\hbar} S_{q}} .
$$

\subsubsection{Path Integrals}

Let us consider the disc $D=\{z \in C|| z \mid \leq 1\}$. Since the Poisson sigma model is invariant under conformal transformations, we map the disc to the upper half-plane $\Sigma=\left\{z=\sigma^{0}+\right.$ $\left.i \sigma^{1} \mid \sigma^{1} \geq 0\right\}$ by a conformal transformation. Then, we consider

$$
S=S_{0}+S_{1}=\int_{T[1] \Sigma} d^{2} \sigma d^{2} \theta\left(\boldsymbol{A}_{i} \boldsymbol{d} \phi^{i}+\frac{1}{2} f^{i j}(\boldsymbol{\phi}) \boldsymbol{A}_{i} \boldsymbol{A}_{j}\right)
$$

where $\boldsymbol{\phi}^{i}=\boldsymbol{x}^{i}$ and $\boldsymbol{A}_{i}=\boldsymbol{\xi}_{i}$.

The partition function $Z=\int_{L} \mathcal{D} \Phi e^{\frac{i}{\hbar} S_{q}}$ and correlation functions are calculated by a formal perturbative expansion in $\hbar$ in the path integral,

$$
Z\left(\mathcal{O}_{1} \cdots \mathcal{O}_{r}\right)=\int_{L} \mathcal{D} \Phi \mathcal{O}_{1} \cdots \mathcal{O}_{r} e^{\frac{i}{\hbar} S_{q}}=\sum_{k=0}^{\infty} \hbar^{k} Z_{k}\left(\mathcal{O}_{1} \cdots \mathcal{O}_{r}\right)
$$

Here, $S_{q}$ is the gauge fixed quantum action and the $\mathcal{O}_{s}$ are observables.

Since a complete superfield formalism is not known for gauge fixed actions of AKSZ theories, we expand it in the component fields. The superfields are expanded in $\theta^{\mu}$ as follows,

$$
\begin{aligned}
& \phi^{i}=\phi^{i}+A^{+i}+c^{+i}, \\
& \boldsymbol{A}_{i}=-c_{i}+A_{i}+\phi_{i}^{+} .
\end{aligned}
$$




\subsubsection{BV Quantization}

In general, the gauge symmetry algebra of an AKSZ sigma model is an open algebra. Thus, we apply the BV quantization procedure [61, 52]. We consider the gauge fixing of the action $S$.

First, we introduce an FP antighost $\bar{c}^{i}$ of ghost number gh $\bar{c}^{i}=-1$, a Nakanishi-Lautrup multiplier field $b^{i}$ of gh $b^{i}=0$ and their antifields $\bar{c}_{i}^{+}=\frac{1}{2} \theta^{\mu} \theta^{\nu} \bar{c}_{\mu \nu i}^{+}$of $\operatorname{gh} \bar{c}_{i}^{+}=0$ and $b_{i}^{+}=$ $\frac{1}{2} \theta^{\mu} \theta^{\nu} b_{\mu \nu i}^{+}$of gh $b_{i}^{+}=-1$. Then, the P-structure (antibracket) is extended as

$$
\left\{\bar{c}^{i}, \bar{c}_{j}^{+}\right\}=\left\{b^{i}, b_{j}^{+}\right\}=\delta^{i}{ }_{j}
$$

and the other antibrackets are zero.

The following gauge fixing term is added to the classical BV action $S$,

$$
S_{G F}=-\int_{T[1] \Sigma} d^{2} \sigma d^{2} \theta b^{i} \bar{c}_{i}^{+},
$$

and we denote $S_{q}=S+S_{G F}$.

Next, the gauge fixing fermion $\Psi(\Phi)$ of ghost number one is determined such that it restricts the path integral to the subspace of the gauge fixed fields and ghosts. We take the gauge fixing fermion as

$$
\Psi=\int_{T[1] \Sigma} d^{2} \sigma d^{2} \theta \bar{c}^{i} \boldsymbol{d} * A_{i},
$$

where $*$ is the Hodge star on $\Sigma$. The BV gauge fixing is carried out by imposing the following equation,

$$
\Phi^{+}=\frac{\delta \Psi}{\delta \Phi}
$$

All the antifields are fixed by this gauge fixing condition. In components, we obtain

$$
\begin{aligned}
& \bar{c}_{i}^{+}=\boldsymbol{d} * A_{i}, \quad A^{+i}=* \boldsymbol{d} \bar{c}^{i}, \\
& \phi_{i}^{+}=0, \quad c_{i}^{+}=b_{i}^{+}=0 .
\end{aligned}
$$

Substituting equations (12.136) into the BV action $S_{q}$, we obtain the gauge fixed quantum BV action, $S_{q \mid f i x}(\Phi)=S_{q}\left(\Phi, \Phi^{+}=\frac{\delta \Psi}{\delta \Phi}\right)$ :

$$
\begin{aligned}
S_{q \mid f i x}= & \int_{T[1] \Sigma} d^{2} \sigma d^{2} \theta\left(A_{i} \boldsymbol{d} \phi^{i}-* \boldsymbol{d} \bar{c}^{i} \boldsymbol{d} c_{i}-b^{i} \boldsymbol{d} * A_{i}+\frac{1}{2} f^{i j}(\phi) A_{i} A_{j}-\right. \\
& \left.-\frac{\partial f^{i j}}{\partial \phi^{k}}(\phi) * \boldsymbol{d} \bar{c}^{k} A_{i} c_{j}+\frac{1}{4} \frac{\partial^{2} f^{i j}}{\partial \phi^{k} \partial \phi^{l}}(\phi) * \boldsymbol{d} \bar{c}^{k} * \boldsymbol{d} \bar{c}^{l} c_{i} c_{j}\right) .
\end{aligned}
$$


The partition function $Z$ must be independent of the gauge fixing conditions. This means that the partition function is invariant under arbitrary infinitesimal changes of the gauge fixing fermion $\Psi$,

$$
Z(\Psi)=Z(\Psi+\delta \Psi)
$$

This requirement gives the following consistency condition for the quantum BV action $S_{q}=$ $S+S_{G F}$,

$$
\Delta e^{\frac{i}{\hbar} S_{q}\left(\Phi, \Phi^{+}\right)}=0
$$

where $\Delta$ is the odd Laplace operator (6.85) introduced in Section 6. This equation is equivalent to the quantum master equation,

$$
2 i \hbar \Delta S_{q}-\left\{S_{q}, S_{q}\right\}=0
$$

We can prove that the AKSZ sigma models formally satisfy this equation. More precisely, the AKSZ sigma models satisfy $\Delta S_{q}=0$ and $\left\{S_{q}, S_{q}\right\}=0$. Since these equations contain divergences in general, we need to renormalize in order to prove these equations beyond the

formal expressions. As we discuss later, we can properly regularize the equation in the Poisson sigma model.

The correlation function of an observable $\mathcal{O}$,

$$
\langle\mathcal{O}\rangle=\int_{\Phi^{+}=\frac{\delta \Psi}{\delta \Phi}} \mathcal{D} \Phi \mathcal{O} e^{\frac{i}{\hbar} S_{q}},
$$

must also be invariant under infinitesimal changes of the gauge fixing fermion $\Psi$. This condition is equivalent to

$$
\Delta\left(\mathcal{O} e^{\frac{i}{\hbar} S_{q}}\right)=0
$$

and can be rewritten as

$$
i \hbar \Delta \mathcal{O}-\left\{S_{q}, \mathcal{O}\right\}=0
$$

\subsubsection{Boundary Conditions}

Here, we determine the boundary conditions of the classical theory, using the same procedure as explained in Section 10. 
The boundary conditions on each field are determined by two consistency conditions. The variation of the action is

$$
\delta S=\int_{T[1] \Sigma} d^{2} \sigma d^{2} \theta\left(\delta \boldsymbol{A}_{i} \boldsymbol{d} \phi^{i}+\boldsymbol{A}_{i} \boldsymbol{d} \delta \boldsymbol{\phi}^{i}+\delta \boldsymbol{\phi}^{i} \frac{1}{2} \frac{\partial f^{j k}}{\partial \boldsymbol{\phi}^{i}}(\boldsymbol{\phi}) \boldsymbol{A}_{j} \boldsymbol{A}_{k}+f^{i j}(\boldsymbol{\phi}) \delta \boldsymbol{A}_{i} \boldsymbol{A}_{j}\right) .
$$

In order to obtain the equations of motion, we need to integrate the second term $\boldsymbol{A}_{i} \boldsymbol{d} \delta \boldsymbol{\phi}^{i}$ by parts. Its boundary integral must vanish. Thus, we obtain

$$
\begin{aligned}
\int_{T[1] \Sigma} d^{2} \sigma d^{2} \theta \boldsymbol{d}\left(\boldsymbol{A}_{i} \delta \phi^{i}\right) & =\int_{\partial T[1] \Sigma} d \sigma^{0} d \theta^{0} \boldsymbol{A}_{i} \delta \phi^{i} \\
& =\int_{\partial T[1] \Sigma} d \sigma^{0} d \theta^{0}\left(A_{i} \delta \phi^{i}-c_{i} \delta A^{+i}\right)=0 .
\end{aligned}
$$

The possible boundary conditions that satisfy equation (12.143) are $A_{/ / i} \mid=0$ or $\delta \phi^{i} \mid=0$, and $c_{i} \mid=0$ or $\delta A_{/ /}^{+i} \mid=0{ }^{\mu}$ Here, the notation $A_{/ / i}=A_{0 i}$ means the component parallel to the boundary and $\Phi \mid$ denotes the value of $\Phi$ on the boundary. In order to obtain a nontrivial solution for the embedding map from $\Sigma$ to $M, \phi^{i}$, we take $A_{/ / i} \mid=0$ and $c_{i} \mid=0$.

The classical equations of motion are

$$
\begin{aligned}
& \boldsymbol{d} \phi^{i}+f^{i j}(\boldsymbol{\phi}) \boldsymbol{A}_{j}=0, \\
& \boldsymbol{d} \boldsymbol{A}_{i}+\frac{1}{2} \frac{\partial f^{j k}}{\partial \phi^{i}}(\boldsymbol{\phi}) \boldsymbol{A}_{j} \boldsymbol{A}_{k}=0 .
\end{aligned}
$$

From the equations of motion and the boundary conditions, $A_{/ / i} \mid=0$ and $c_{i} \mid=0$, we obtain the boundary conditions $\phi^{i} \mid=$ constant and $A_{/ /}^{+i} \mid=0$. Therefore, the boundary conditions for all fields are

$$
\begin{aligned}
& \phi^{i} \mid=x^{i}=\text { constant }, \quad A_{/ / i} \mid=0, \\
& c_{i}\left|=0, \quad A_{/ /}^{+i}\right|=0 .
\end{aligned}
$$

Here $x^{i}$ parametrize the boundary.

Next, we determine the boundary conditions for other extra fields. The consistency conditions for the equations of motion of the gauge fixed action (12.137) fix the boundary conditions for the ghost $b^{i}=0$. The boundary conditions for the other ghosts and antifields are determined by consistency with the gauge fixing conditions of equation (12.136) as

$$
\begin{aligned}
& \phi_{i}^{+}\left|=0, \quad c_{i}^{+}\right|=b_{i}^{+} \mid=0, \\
& \bar{c}_{i}^{+}\left|=\boldsymbol{d} * A_{i}\right|, \quad \bar{c}^{i} \mid=\text { constant. }
\end{aligned}
$$

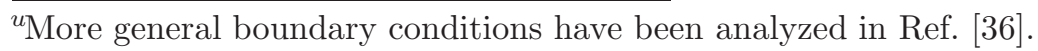


These boundary conditions are consistent with the master equation.

\subsubsection{Propagators}

The propagators are defined by the first three terms of the gauge fixed action (12.137),

$$
\begin{aligned}
S_{F} & =\int_{T[1] \Sigma} d^{2} \sigma d^{2} \theta\left(A_{i} \boldsymbol{d} \phi^{i}-b^{i} \boldsymbol{d} * A_{i}-* \boldsymbol{d} \bar{c}^{i} \boldsymbol{d} c_{i}\right) \\
& =\int_{T[1] \Sigma} d^{2} \sigma d^{2} \theta\left(A_{i} \boldsymbol{d} \phi^{i}+A_{i} * \boldsymbol{d} b^{i}-c_{i} \boldsymbol{d} * \boldsymbol{d} \bar{c}^{i}\right) .
\end{aligned}
$$

If we introduce the gauge fixed superfields,

$$
\begin{aligned}
& \boldsymbol{\varphi}^{i}=\varphi^{i}+* \boldsymbol{d} \bar{c}^{i}+0 \\
& \boldsymbol{A}_{i}=-c_{i}+A_{i}+0
\end{aligned}
$$

the propagators of each component field are combined to a superfield propagator, where $\varphi$ is defined by $\phi^{i}=x^{i}+\varphi^{i}$.

Let $\boldsymbol{d}_{z}$ and $\boldsymbol{d}_{w}$ be superderivatives with respect to the variables $z$ and $w$. Let $G(z, w)$ be a Green's function such that $\boldsymbol{d}_{w} * \boldsymbol{d}_{w} G(z, w)=2 \pi \delta(z-w)$, where $G(z, w)$ is determined by the Dirichlet boundary condition for $z$ and the Neumann boundary condition for $w$, respectively.

The solution is $G(z, w)=\frac{1}{2 i} \ln \frac{(z-w)(z-\bar{w})}{(\bar{z}-\bar{w})(\bar{z}-w)}$. Using this Green's function, the superpropagator of $\left(\varphi^{i}, \boldsymbol{d} \bar{c}^{i}, c_{i}, A_{i}\right)$ is determined as

$$
\left\langle\boldsymbol{\varphi}^{i}(w) \boldsymbol{A}_{j}(z)\right\rangle=\frac{i \hbar}{2 \pi} \delta^{i}{ }_{j}\left(\boldsymbol{d}_{z}+\boldsymbol{d}_{w}\right) G(z, w)
$$

which is consistent with the boundary conditions for each field. In addition to equation (12.147), there is the propagator of $A_{i}$ and $b^{i}$, which we omit, since the star product does not involve the propagator $\left\langle A_{i}(w) b^{j}(z)\right\rangle$. 


\subsubsection{Vertices}

The last three terms of the gauge fixed action (12.137) are interaction terms denoted by $S_{I}$, and they define the vertices. $S_{I}$ is simplified using gauge fixed superfields (12.146) as follows,

$$
\begin{aligned}
S_{I}= & \int_{T[1] \Sigma} d^{2} \sigma d^{2} \theta\left(\frac{1}{2} f^{i j}(\phi) A_{i} A_{j}-\frac{\partial f^{i j}}{\partial \phi^{k}}(\phi) * \boldsymbol{d} \bar{c}^{k} A_{i} c_{j}\right. \\
& \left.+\frac{1}{4} \frac{\partial^{2} f^{i j}}{\partial \phi^{k} \partial \phi^{l}}(\phi) * \boldsymbol{d} \bar{c}^{k} * \boldsymbol{d} \bar{c}^{l} c_{i} c_{j}\right) \\
= & \int_{T[1] \Sigma} d^{2} \sigma d^{2} \theta \frac{1}{2} f^{i j}(\boldsymbol{\phi}) \boldsymbol{A}_{i} \boldsymbol{A}_{j} .
\end{aligned}
$$

In order to identify the vertices, $\boldsymbol{\phi}^{i}$ and $\boldsymbol{A}_{i}$ are expanded around the classical solutions $\phi^{i}=x^{i}+\varphi^{i}$ and $\boldsymbol{A}_{i}=0+\boldsymbol{A}_{i}$. Taylor expansion of $f^{i j}(\phi)$ gives

$$
S_{I}=\frac{1}{2} \int_{T[1] \Sigma} d^{2} \sigma d^{2} \theta \sum_{k=0}^{\infty} \frac{1}{k !} \partial_{l_{1}} \partial_{l_{2}} \cdots \partial_{l_{k}} f^{i j}(x) \boldsymbol{\varphi}^{l_{1}} \boldsymbol{\varphi}^{l_{2}} \cdots \boldsymbol{\varphi}^{l_{k}} \boldsymbol{A}_{i} \boldsymbol{A}_{j}
$$

which determines the vertices of order $\hbar^{-1}$. Note that there is an infinite number of vertices. From equation (12.149), the $k$-th vertex has two $\boldsymbol{A}$ lines and $k \boldsymbol{\varphi}$ lines that have the weight $\frac{1}{2} \frac{1}{k !} \partial_{l_{1}} \partial_{l_{2}} \cdots \partial_{l_{k}} f^{i j}(x)$.

The path integral of an observable $\mathcal{O}$ can be expanded as

$$
\langle\mathcal{O}\rangle=\int \mathcal{O} e^{\frac{i}{\hbar}\left(S_{F}+S_{I}\right)}=\sum_{n=0}^{\infty} \frac{i^{n}}{\hbar^{n} n !} \int \mathcal{O} e^{\frac{i}{\hbar} S_{F}} S_{I}^{n} .
$$

Since $\mathcal{O}$ is a function of superfields $\boldsymbol{\varphi}$ and $\boldsymbol{A}$, it is computed by Wick's theorem using the propagators $\left\langle\boldsymbol{\varphi}^{i}(w) \boldsymbol{A}_{j}(z)\right\rangle$, as in usual perturbation theory.

\subsubsection{Renormalization of Tadpoles}

Contributions from tadpoles are renormalized to zero in order to derive a star product. Although this renormalization is different from the one usually used in quantum field theory, it can be carried out consistently with the quantum master equation. We can add a gauge invariant counter term that subtracts all tadpole contributions,

$$
S_{c t}=\int_{T[1] \Sigma} d^{2} \theta d^{2} \sigma \frac{\partial f^{i j}(\boldsymbol{\phi})}{\partial \phi^{i}} \boldsymbol{A}_{j} \kappa,
$$

where $\kappa$ is the subtraction coefficient of the renormalization. 


\subsubsection{Correlation Functions of Observables on the Boundary}

An arbitrary function of $\phi, F(\phi)$, restricted to the boundary of $\Sigma$, is an observable since it satisfies equation (12.141). We now compute the correlation functions of these observables (often called vertex operators). They satisfy the first condition in the definition of a deformation quantization, Definition 12.1.

We consider an observable $\mathcal{O}=F(\phi(t)) G(\phi(s))$ which depends on two points, where $t$ and $s$ are coordinates on the boundary $\partial \Sigma$ and $F$ and $G$ are arbitrary functions of $\phi$. The conformal transformation of the disc worldsheet fixes the three points $0,1, \infty$ on the boundary circle $S^{1}$. The boundary condition of $\phi$ is fixed at $\sigma^{0}=\infty$ as $\phi^{i}(\infty)=x^{i}$, and $\mathcal{O}$ can be transformed to $\mathcal{O}=F(\phi(1)) G(\phi(0))$ by conformal transformation.

We compute the correlation function $\langle F(\phi(1)) G(\phi(0))\rangle$ by the Feynman rules. The order $\hbar^{n}$ amplitudes consist of $n$ vertices and $2 n$ propagators. We choose $n+2$ points on $\Sigma$. There are two points $z=u_{L}=0$ and $z=u_{R}=1$ on the boundary where two vertex operators $F(\phi(1))$ and $G(\phi(0))$ are inserted. Other $n$ points are located in the interior of $\Sigma$. These points are denoted by $u_{j} \in \Sigma,(j=1,2, \cdots, n, L, R)$, where $u_{j}$ for $j=1,2, \cdots, n$ are the points of $n$ vertices. A propagator $d G(z, w)$ connects two points chosen from the above $n+2$ points. We introduce a map $v_{a}:\{1,2, \cdots, n\} \rightarrow\{1,2, \cdots, n, L, R\}$, where $a=1,2$, and $d G\left(u_{j}, u_{v_{a}(j)}\right)$ denotes the propagator from $u_{j}$ to $u_{v_{a}(j)}$, where $j=1,2, \cdots, n$, since two vertex operators on the boundary are functions of $\phi . v_{a}(j) \neq j$ for all $j$, since we renormalize the tadpole graphs to zero as in Section 12.1.7. Since all the vertices contain precisely two $\boldsymbol{A}_{i}$ 's, the weight of the nonzero Feynman diagram is obtained as

$$
\frac{1}{n !}\left(\frac{(i \hbar)^{n}}{(2 \pi)^{2 n}}\right) \int \wedge_{j=1}^{n} \boldsymbol{d} G\left(u_{j}, u_{v_{1}(j)}\right) \wedge \boldsymbol{d} G\left(u_{j}, u_{v_{2}(j)}\right),
$$

where $\boldsymbol{d}=\boldsymbol{d}_{z}+\boldsymbol{d}_{w}$. This gives coefficients of the $\hbar^{n}$ term of the star product $(-1)^{n} \mathcal{B}_{\Gamma n}(F, G)$ induced from the Feynman diagram $\Gamma$.

The first two terms of the perturbative expansion are

$$
\begin{aligned}
\langle F(\phi(1)) G(\phi(0))\rangle & =\int_{\phi(\infty)=x} \mathcal{D} \Phi F(\phi(1)) G(\phi(0)) e^{\frac{i}{\hbar} S_{q}} \\
& =F(x) G(x)+\frac{i \hbar}{2} f^{i j}(x) \frac{\partial F(x)}{\partial x^{i}} \frac{\partial G(x)}{\partial x^{j}}+O\left(\hbar^{2}\right) \\
& =F(x) G(x)+\frac{i \hbar}{2}\{F(x), G(x)\}_{P B}+O\left(\hbar^{2}\right),
\end{aligned}
$$


where the first term is the solution of the classical equations of motion and the second term is the Poisson bracket of $F$ and $G$. This correlation function satisfies the first condition in Definition 12.1.

Higher-order terms are determined by the Feynman diagrams. From equation (12.151), the Poisson sigma model has been determined only by the Poisson structure on $M$, and thus higher-order terms in the expansion are expressed by $f^{i j}$ and its derivatives.

If $f^{i j}(x)$ is a constant, the perturbation is simplified at all orders. In this case, (12.149) has one vertex without derivatives of $f, \frac{1}{2} f^{i j}(x) \boldsymbol{A}_{i} \boldsymbol{A}_{j}$. Therefore, we obtain

$$
\begin{aligned}
\langle F(\phi(1)) G(\phi(0))\rangle & =\int_{\phi(\infty)=x} \mathcal{D} \Phi F(\phi(1)) G(\phi(0)) e^{\frac{i}{\hbar} S_{q}} \\
& =\sum_{n=0}^{\infty} \lim _{y \rightarrow x} \exp \left(\frac{i \hbar}{2} f^{i j} \frac{\partial}{\partial x^{i}} \frac{\partial}{\partial y^{j}}\right)^{n} F(x) G(y) .
\end{aligned}
$$

This is nothing but the Moyal product, which is the star product derived from the constant antisymmetric tensor $f^{i j}$.

\subsubsection{Associativity and Equivalence}

In this section, we explain how the correlation function (12.151) satisfies Condition (2) of Definition 12.1, i.e., the associativity condition.

The associativity condition is derived from the Ward-Takahashi identity of the gauge symmetry of this theory. In the BV formalism, the Ward-Takahashi identity is derived from the quantum master equation (12.138) and its path integral,

$$
\int_{\phi(\infty)=x} \mathcal{D} \Phi \Delta\left(\mathcal{O} e^{\frac{i}{\hbar} S_{q}}\right)=0
$$

Take an observable $\mathcal{O}=F(\phi(1)) G(\phi(t)) H(\phi(0))$ on the boundary, where $t$ is a coordinate on the boundary such that $0<t<1$, and let $\tau$ be a supercoordinate partner of $t$. Since the conformal transformation in two dimensions fixes only three points, this observable has the modulus $t$. Substituting this observable into equation (12.152), we get

$$
\int_{\phi(\infty)=x, 1>t>0} d t d \tau \mathcal{D} \Phi \Delta\left(F(\phi(1)) G(\phi(t)) H(\phi(0)) e^{\frac{i}{\hbar} S_{q}}\right)=0 .
$$

From equations (12.138) and (12.152), we obtain

$$
\int_{\phi(\infty)=x, 1>t>0} d t d \tau \mathcal{D} \Phi\left\{S_{q}, F(\phi(1)) G(\phi(t)) H(\phi(0))\right\} e^{\frac{i}{\hbar} S_{q}}=0 .
$$


Substituting

$$
\left\{S_{q}, F(\phi(1)) G(\phi(t)) H(\phi(0))\right\}=-\boldsymbol{d}(F(\phi(1)) G(\phi(t)) H(\phi(0)))
$$

and applying Stokes' theorem, this path integral becomes a boundary integral on the moduli space,

$$
\begin{aligned}
& \lim _{t \rightarrow 1} \int_{\phi(\infty)=x} \mathcal{D} \Phi\left(F(\phi(1)) G(\phi(t)) H(\phi(0)) e^{\frac{i}{\hbar} S_{q}}\right) \\
& -\lim _{t \rightarrow 0} \int_{\phi(\infty)=x} \mathcal{D} \Phi\left(F(\phi(1)) G(\phi(t)) H(\phi(0)) e^{\frac{i}{\hbar} S_{q}}\right)=0 .
\end{aligned}
$$

This equation leads to the associativity relation

$$
(F * G) * H-F *(G * H)=0
$$

for $F, G, H \in C^{\infty}(M)[[\hbar]]$.

Next, we discuss Condition (3) in Definition 12.1. It is sufficient to prove the following statement: Let $F(x)$ be a function such that $\{F(x), G(x)\}_{P B}=0$ for any $G$. Then, $F * G(x)$ is equivalent to the normal product $F(x) G(x)$ by a redefinition $F^{\prime}=R F$

If $\{F(x),-\}_{P B}=0, F(\phi(u)) G(\phi(0))$ is an observable, where $u$ is an interior point on the disc. Thus, the correlation function

$$
\langle F(\phi(u)) G(\phi(0))\rangle=\int_{\phi(\infty)=x} \mathcal{D} \Phi F(\phi(u)) G(\phi(0)) e^{\frac{i}{\hbar} S_{q}}
$$

satisfies the following Ward-Takahashi identity,

$$
\int_{\phi(\infty)=x} \mathcal{D} \Phi \Delta\left(F(\phi(u)) G(\phi(0)) e^{\frac{i}{\hbar} S_{q}}\right)=0 .
$$

From equation (12.155) and a similar computation to the derivation of (12.153) using $\{S, F(\phi(u))\}=$ $\boldsymbol{d} F(\phi(u))$, we obtain

$$
\int_{\phi(\infty)=x} \mathcal{D} \Phi \boldsymbol{d} F(\phi(u)) G(\phi(0)) e^{\frac{i}{\hbar} S_{q}}=0 .
$$

This means that the correlation function $\langle F(\phi(u)) G(\phi(0))\rangle$ is independent of $u$.

For $G=0$, we obtain the one-point function,

$$
\langle F(\phi(u))\rangle=\int_{\phi(\infty)=x} \mathcal{D} \Phi F(\phi(u)) e^{\frac{i}{\hbar} S_{q}}=F(x)+O\left(\hbar^{2}\right),
$$

$\overline{{ }^{v} \text { Note that if }\{F, G\}_{P B}=0 \text {, then } F * G(x)}=F(x) G(x)$ is a trivial solution of the deformation quantization. 
which is expressed by a formal series of derivatives of $F(x)$ as $\sum_{k}\left(\frac{i \hbar}{2}\right)^{k} \mathcal{D}_{k}(F)$. Then, we can take $R F(x)=\langle F(\phi(u))\rangle$.

We can prove that

$$
R F * G(x)=\langle F(\phi(1)) G(\phi(0))\rangle=\lim _{\epsilon \rightarrow+0}\langle F(\phi(1+i \epsilon)) G(\phi(0))\rangle
$$

by the factorization property of the path integral. This shows that $R F * G(x)$ is equivalent to $F(x) G(x)$.

\subsection{Formality}

The mathematical proof of the existence of a deformation quantization on a Poisson manifold [93, 33] is called the formality theorem, and it is closely related to the quantization of the Poisson sigma model. In this article, we discuss the correspondence between mathematical terms and physical concepts appearing in the AKSZ sigma model.

\subsubsection{Differential Graded Lie Algebras}

The input data of the deformation quantization is a Poisson bracket $\{F, G\}_{P B}$. As we saw in Example 5.2.1, the Poisson structure can be interpreted in terms of supergeometry. Thus, a deformation quantization is also reformulated in terms of supergeometry or graded algebras. First, we introduce a differential graded Lie algebra.

Definition 12.3 A differential graded Lie algebra (dg Lie algebra) $(\mathfrak{g},\{-,-\}, d)$ is a graded algebra with $\mathbb{Z}$-degree $\mathfrak{g}=\oplus_{k \in \mathbb{Z}} \mathfrak{g}^{k}[-k]$, where $\mathfrak{g}^{k}$ is the degree $k$ part of $\mathfrak{g} .\{-,-\}: \mathfrak{g}^{k} \times \mathfrak{g}^{l} \rightarrow$ $\mathfrak{g}^{k+l}$ is a graded Lie bracket and $d: \mathfrak{g}^{k} \longrightarrow \mathfrak{g}^{k+1}$ is a differential of degree 1 such that $d^{2}=0$.

\subsubsection{Maurer-Cartan Equations of Poisson Bivector Fields}

We consider a QP-manifold of degree $1,(\mathcal{M}, \omega, \Theta)$. The graded Poisson bracket, $\{-,-\}$, induced by the P-structure is identified with the graded Lie bracket of the dg Lie algebra, where the degree is shifted by 1 . The corresponding differential is $d=0$. The space of functions of degree 2 in $C^{\infty}(\mathcal{M})$ is identified with $\mathfrak{g}^{1}$, which is isomorphic to the space of the bivector fields, $\alpha_{1}=\frac{1}{2} \alpha^{i j}(x) \partial_{i} \wedge \partial_{j}$. Then, the space $\left(\mathfrak{g}^{1}=\Gamma\left(\wedge^{2} T M\right),\{-,-\}, d=0\right)$ is a $\mathrm{dg}$ Lie algebra and denoted by $\mathfrak{g}_{1}^{1}=T_{\text {poly }}^{1}(M)$. 
Next, we consider the subspace of the solutions of the Maurer-Cartan equation $d \alpha_{1}+$ $\frac{1}{2}\left\{\alpha_{1}, \alpha_{1}\right\}=0$ in $\mathfrak{g}_{1}^{1}$. This space is denoted by $\mathcal{M C}\left(\mathfrak{g}_{1}^{1}\right)=\mathfrak{g}_{1}^{1} / \sim$. It is equivalent to the solutions of the classical master equation $\{\Theta, \Theta\}=0$ since $d=0$ and $\Theta$ is of degree 2 and can be identified with a bivector field. Therefore, the QP-manifold of degree $1,(\mathcal{M}, \omega, \Theta)$, is identified with $\mathcal{M C}\left(\mathfrak{g}_{1}^{1}\right)$.

\subsubsection{Hochschild Complex of Polydifferential Operators}

The $\hbar^{1}$-th order of the deformation quantization corresponds to the classical theory in physics.

The Poisson bivector $\alpha_{1}=\frac{1}{2} f^{i j}(x) \partial_{i} \wedge \partial_{j}$ determines first two terms of the star product as $(F, G) \mapsto \mathcal{B}_{0}(F, G)+\frac{i \hbar}{2} \mathcal{B}_{1}(F, G)=F G+\frac{i \hbar}{2} \frac{1}{2} f^{i j}(x) \partial_{i} F \partial_{j} G \in \operatorname{Hom}\left(A^{\otimes 2}, A\right)$, where $F, G \in A=$ $C^{\infty}(M)$.

From Condition (3) in Definition 12.1, the two expressions of $\mathcal{B}_{0}(F, G)+\frac{i \hbar}{2} \mathcal{B}_{1}(F, G)$ and $\mathcal{B}_{0}\left(F^{\prime}, G\right)+\frac{i \hbar}{2} \mathcal{B}_{1}\left(F^{\prime}, G\right)$ are equivalent in $\hbar^{1}$-th order, if they coincide after $F$ is redefined as $F^{\prime}=F+\frac{i \hbar}{2} \mathcal{D} F$. The redefinition map is an element of $\operatorname{Hom}(A, A)$.

In order to prove associativity, we must consider a map $C(F, G, H)$ in $\operatorname{Hom}\left(A^{\otimes 3}, A\right)$. The following associativity relation is obtained at classical level, i.e., at $\hbar^{1}$-th order,

$$
\begin{aligned}
C:(F, G, H) \mapsto & C(F, G, H) \\
& =(F G) H-F(G H) \\
& +\frac{i \hbar}{2}\left(\mathcal{B}_{1}(F G, H)-\mathcal{B}_{1}(F, G H)+\mathcal{B}_{1}(F, G) H-F \mathcal{B}_{1}(G, H)\right) \\
& +\left(\frac{i \hbar}{2}\right)^{2}\left(\mathcal{B}_{1}\left(\mathcal{B}_{1}(F, G), H\right)-\mathcal{B}_{1}\left(F, \mathcal{B}_{1}(G, H)\right)\right) .
\end{aligned}
$$

The classical associativity holds, if

$$
C(F, G, H)=0
$$

To formulate associativity for all orders in $\hbar$, we define a second dg Lie algebra in $\operatorname{Hom}\left(A^{\otimes k+1}, A\right)$. Let $\mathfrak{g}_{2}=\oplus_{k \in \mathbb{Z}, k \geq-1} \mathfrak{g}_{2}^{k}[-k]$, where $\mathfrak{g}_{2}^{k}=\operatorname{Hom}\left(A^{\otimes k+1}, A\right)$. For an element $C \in \mathfrak{g}_{2}^{k}$, a differential $d$ and a graded Lie bracket $[-,-]$ are defined in such a way that equation (12.159) is obtained as a part of the Maurer-Cartan equation. The differential is defined 
as

$$
\begin{aligned}
(d C)\left(F_{0} \otimes \cdots \otimes F_{k+1}\right)= & F_{0} C\left(F_{1} \otimes \cdots \otimes F_{k+1}\right)-\sum_{r=0}^{k} C\left(F_{0} \otimes \cdots \otimes\left(F_{r} F_{r+1}\right) \otimes \cdots \otimes F_{k+1}\right) \\
& +(-1)^{k} C\left(F_{0} \otimes \cdots \otimes F_{k}\right) F_{k+1} .
\end{aligned}
$$

The graded Lie bracket is defined as

$$
\begin{gathered}
{\left[C_{1}, C_{2}\right]=C_{1} \circ C_{2}-(-1)^{k_{1} k_{2}} C_{2} \circ C_{1},} \\
C_{1} \circ C_{2}\left(F_{0} \otimes \cdots \otimes F_{k_{1}+k_{2}}\right)=\sum_{r=0}^{k}(-1)^{r k} C_{1}\left(F_{0} \otimes \cdots \otimes F_{r-1} \otimes C_{2}\left(F_{r} \otimes \cdots \otimes F_{r+k_{2}}\right)\right. \\
\left.\otimes F_{r+k_{2}+1} \otimes \cdots \otimes F_{k_{1}+k_{2}}\right),
\end{gathered}
$$

where $C_{1} \in \mathfrak{g}_{2}^{k_{1}}$ and $C_{2} \in \mathfrak{g}_{2}^{k_{2}}$. Note that $\left(\mathfrak{g}_{2}, d\right)$ is called the Hochschild complex of polydifferential operators, and is also denoted as $\mathfrak{g}_{2}^{k}=D_{\text {poly }}^{k}(M)$ and $\mathfrak{g}_{2}=D_{\text {poly }}(M)$. The bracket $[-,-]$ is called the Gerstenhaber bracket.

For an element $\widetilde{\alpha} \in \mathfrak{g}_{2}^{1}$ of degree 1 , the Maurer-Cartan equation $d \widetilde{\alpha}+\frac{1}{2}[\widetilde{\alpha}, \widetilde{\alpha}]=0$ is equivalent to the associativity equation (12.159). Equivalence under redefinition, Condition (3), is also expressed by the Maurer-Cartan equation in elements on $\mathfrak{g}_{2}^{0}$. Therefore, a solution of the Maurer-Cartan equation in $\mathfrak{g}_{2}$ gives the star product at order $\hbar^{1}$. The space of solutions of the Maurer-Cartan equation is denoted by $\mathcal{M C}\left(\mathfrak{g}_{2}\right)=\mathfrak{g}_{2} / \sim$.

\subsubsection{Morphisms of Two Differential Graded Lie Algebras}

At classical level, i.e., at $\hbar^{1}$-th order, we define a map $U_{1}: \mathfrak{g}_{1}^{1} \longrightarrow \mathfrak{g}_{2}^{1}$, such that $U_{1}: \frac{1}{2} f^{i j}(x) \partial_{i} \wedge$ $\partial_{j} \mapsto\left(F_{0} \otimes F_{1} \mapsto \frac{1}{2} f^{i j}(x) \partial_{i} F_{0} \partial_{j} F_{1}\right)$. Since this map preserves the Maurer-Cartan equations, this induces the map $U_{1}: \mathcal{M C}\left(\mathfrak{g}_{1}^{1}\right) \longrightarrow \mathcal{M C}\left(\mathfrak{g}_{2}^{1}\right)$.

A deformation quantization is expressed as follows. Fix the map $U_{1}$. The problem is to find a morphism on $\hbar$ deformations of two dg Lie algebras, $U: \mathcal{M C}\left(\mathfrak{g}_{1}^{1}[[\hbar]]\right) \longrightarrow \mathcal{M C}\left(\mathfrak{g}_{2}[[\hbar]]\right)$.

In general, the Maurer-Cartan equation on $\mathcal{M C}\left(\mathfrak{g}_{2}[[\hbar]]\right)$ is not preserved by a linear deformation of $U_{1}$, since $U_{1}$ does not preserve graded Lie brackets. To find $U$ consistent with the MC equations, we extend the two dg Lie algebras to $L_{\infty}$-algebras. Then, we construct the map $U$ as an $L_{\infty}$-morphism between them.

We extend $\mathfrak{g}_{1}^{1}$ to the space of polyvector fields $T_{\text {poly }}(M)=\mathfrak{g}_{1}=\oplus_{k \in \mathbb{Z}, k \geq-1} \mathfrak{g}_{1}^{k}[-k]$, where $\mathfrak{g}_{1}^{k}=\Gamma\left(\wedge^{k+1} T M\right)$. An element of $\mathfrak{g}_{1}^{k}$ is a $k$-th multivector field (an order $k$ antisymmetric 
tensor field), $\alpha_{k}=\alpha^{j_{0} \cdots j_{k}}(x) \partial_{j_{0}} \wedge \cdots \wedge \partial_{j_{k}}=\alpha^{j_{0} \cdots j_{k}}(x) \xi_{j_{0}} \cdots \xi_{j_{k}} \in \mathfrak{g}_{1}^{k}$. The differential and the graded Lie bracket on $\mathfrak{g}_{1}^{1}$ are generalized to $\mathfrak{g}_{1}$ as follows. The differential is kept trivial, $d=0$, and the graded Lie bracket is the Schouten-Nijenhuis bracket $[-,-]_{S}$ of multivector fields, i.e., the graded Poisson bracket $\{-,-\}$ of degree 1 of the QP-manifold $\mathcal{M}=T^{*}[1] M$.

The map between two dg Lie algebras in the classical theory is defined as follows,

$$
\begin{aligned}
U_{1}: \quad & T_{\text {poly }}(M) \longrightarrow D_{\text {poly }}(M), \\
& \alpha_{k} \mapsto\left(F_{0} \otimes \cdots \otimes F_{k} \mapsto \frac{1}{(k+1) !} \alpha^{j_{0} \cdots j_{k}}(x) \partial_{j_{0}} F_{0} \cdots \partial_{j_{k}} F_{k}\right) .
\end{aligned}
$$

Although this map is not isomorphic, $U_{1}$ induces an isomorphism between the $d$-cohomologies of the two spaces, $T_{\text {poly }}(M)$ and $D_{\text {poly }}(M)$ [146] 小 $^{w}$

\subsection{5 $L_{\infty}$-Algebras and $L_{\infty}$-Morphisms}

A dg Lie algebra is embedded into the more general algebra, an $L_{\infty}$-algebra. In this section, we discuss $L_{\infty}$-algebras and $L_{\infty}$-morphisms to describe the statement of the formality theorem.

For a graded vector space $V=\oplus_{k \in \mathbb{Z}} V^{k}$, we consider a graded commutative tensor algebra, $T(V)=\oplus_{n=1}^{\infty} V^{\otimes n}$, which is a space of the sum of infinite tensor products. On this space, a coassociative and cocommutative coproduct $\triangle$ is defined as

$$
\triangle\left(v_{1}, \cdots, v_{n}\right)=\sum_{\sigma \in \mathfrak{S}} \sum_{k=1}^{n-1} \epsilon(\sigma) \frac{1}{k !(n-k) !}\left(v_{\sigma(1)} \cdots v_{\sigma(k)}\right) \otimes\left(v_{\sigma(k+1)} \cdots v_{\sigma(n)}\right),
$$

where $v_{k} \in T(V)$. Next, we assume the following multilinear maps of degree 1 ,

$$
\begin{aligned}
\mathfrak{l}_{k}: \quad & V^{\otimes k} \longrightarrow V, \\
& \left(v_{1} \otimes \cdots \otimes v_{k}\right) \mapsto \mathfrak{l}_{k}\left(v_{1} \cdots v_{k}\right),
\end{aligned}
$$

and define a codifferential $Q=\sum_{k=1}^{\infty} Q_{k}$ as

$$
Q_{k}\left(v_{1}, \cdots, v_{n}\right)=\sum_{\sigma \in \mathfrak{S}} \epsilon(\sigma) \frac{1}{k !(n-k) !} \mathfrak{l}_{k}\left(v_{\sigma(1)} \cdots v_{\sigma(k)}\right) \otimes v_{\sigma(k+1)} \otimes \cdots \otimes v_{\sigma(n)}
$$

\footnotetext{
${ }^{w}$ By definition, if their cohomologies are isomorphic, two spaces are called quasi-isomorphic. The cohomology on $T_{\text {poly }}(M)$ is trivial because $d=0$.
} 
Definition 12.4 A pair $(V, Q)$ is called an $L_{\infty}$-algebra (a strong homotopy Lie algebra) if $Q^{2}=0 .[130,102]$

The first two operations in $\mathfrak{l}_{k}$ are a differential $\mathfrak{l}_{1}=d$ and a superbracket $\mathfrak{l}_{2}(-,-)=\{-,-\}$. Moreover, a graded differential Lie algebra is embedded by the identification, $\mathfrak{g}^{k-1}[1] \sim V^{k-1}$, and $\mathfrak{l}_{k}=0$, for $k \geq 3{ }^{x}$

We now define an $L_{\infty}$-morphism between two $L_{\infty}$-algebras.

Definition 12.5 A map between two $L_{\infty}$-algebras, $U:\left(V_{1}, Q\right) \longrightarrow\left(V_{2}, Q\right)$, is called a cohomomorphism if the map preserves degree and satisfies $\triangle \circ U=(U \otimes U) \circ \triangle$.

Definition 12.6 A cohomomorphism $U$ between two $L_{\infty}$-algebras is called an $L_{\infty}$-morphism if $U Q=Q U$.

Let us denote $e^{v}=1+v+\frac{1}{2 !} v \otimes v+\frac{1}{3 !} v \otimes v \otimes v+\cdots$ and $\mathfrak{l}_{*}\left(e^{v}\right)=\mathfrak{l}_{1}(v)+\frac{1}{2 !} \mathfrak{l}_{2}(v \otimes v)+\frac{1}{3 !} \mathfrak{l}_{3}(v \otimes$ $v \otimes v)+\cdots$.

Definition 12.7 The Maurer-Cartan equation on an $L_{\infty}$-algebra $(V, Q)$ is $\mathfrak{l}_{*}\left(e^{v}\right)=0$.

The Maurer-Cartan equation $\mathfrak{l}_{*}\left(e^{v}\right)=0$ is equivalent to $Q\left(e^{v}\right)=\mathfrak{l}_{*}\left(e^{v}\right) \otimes e^{v}=0$. If an $L_{\infty}$-algebra is a dg Lie algebra, then $Q\left(e^{v}\right)=0$ is equivalent to the ordinary Maurer-Cartan equation $d \alpha+\frac{1}{2}[\alpha, \alpha]=0$, since $\mathfrak{l}_{k}=0$ for $k \geq 3$, where $v=\alpha$.

If we regard two dg Lie algebras $\mathfrak{g}_{1}$ and $\mathfrak{g}_{2}$ as $L_{\infty}$-algebras, the nonlinear correspondence between the two Maurer-Cartan equations on the two dg Lie algebras becomes transparent. Let $V_{1}=\mathfrak{g}_{1}=T_{\text {poly }}(M)[1]$ and $V_{2}=\mathfrak{g}_{2}=D_{\text {poly }}(M)[1]$. Then, the existence of a deformation quantization can be derived as the special case with $\alpha_{k}=0$ except for $k=2$ if the following theorem is proved.

Theorem 12.8 (formality theorem) [92, 93] There exists an $L_{\infty}$-morphism from $\left(T_{\text {poly }}(M)[1], Q\right)$ to $\left(D_{\text {poly }}(M)[1], Q\right)$ such that $U_{1}$ is the map in equation (12.162).

We refer to Ref. 93 for the rigorous proof. In this article, we observe that a two-dimensional AKSZ sigma model contains all the structures required above to find the formality map.

${ }^{x} \mathrm{~A}$ set of functions of a QP-manifold is regarded as an $L_{\infty}$-algebra, where degree of a function on the QPmanifold is equal to degree as an element of the $L_{\infty}$-algebra. 


\subsubsection{Correspondence to $n=1$ AKSZ Sigma Model}

The field theoretical realization of the Poisson structure is the Poisson sigma model, and that of the Maurer-Cartan equations of a dg Lie algebra is the quantum BV master equations. (The MC equation with $d=0$ corresponds to the classical master equation.) The deformation of a dg Lie algebra in $\hbar$ corresponds to the perturbative quantization of a physical theory. The subalgebra $\mathcal{M C}(\mathfrak{g})$ corresponds to the space of correlation functions which satisfy the Ward-Takahashi identities induced from the quantum master equation.

In order to generalize the Poisson sigma model to the $L_{\infty}$ setting, we have to consider the AKSZ sigma model where the target space is generalized to the space of multivector fields, $\mathfrak{g}_{1}$. The BV action of the AKSZ sigma model based on multivector fields is

$$
\begin{aligned}
S & =S_{0}+\sum_{p=0}^{d-1} S_{\alpha_{p}} \\
& =\int_{T[1] \Sigma} d^{2} \sigma d^{2} \theta\left(\boldsymbol{A}_{i} \boldsymbol{d} \phi^{i}+\sum_{p=0}^{d-1} \frac{1}{(p+1) !} \alpha^{j_{0} \cdots j_{p}}(\boldsymbol{\phi}) \boldsymbol{A}_{j_{0}} \cdots \boldsymbol{A}_{j_{p}},\right),
\end{aligned}
$$

where $\alpha_{p}=\frac{1}{(p+1) !} \alpha^{j_{0} \cdots j_{p}}(x) \frac{\partial}{\partial x^{j_{0}}} \wedge \cdots \wedge \frac{\partial}{\partial x^{j_{p}}} \in \Gamma\left(\wedge^{p+1} T M\right)$ is a multivector field satisfying the $\mathrm{MC}$ equation in $\mathcal{M C}\left(\mathfrak{g}_{1}\right)$. We denote the term of the order $p$ multivector field by

$$
S_{\alpha_{p}}=\int_{T[1] \Sigma} d^{2} \sigma d^{2} \theta \frac{1}{(p+1) !} \alpha^{j_{0} \cdots j_{p}}(\boldsymbol{\phi}) \boldsymbol{A}_{j_{0}} \cdots \boldsymbol{A}_{j_{p}},
$$

and $\alpha^{j_{0} j_{1}}(\phi)=f^{j_{0} j_{1}}(\phi)$ corresponds to the original Poisson bivector field. This action $S$ no longer has degree 0 . The $\mathrm{MC}$ equation on $\mathcal{M C}\left(\mathfrak{g}_{1}\right)$ is equivalent to the classical master equation $\{S, S\}=0$.

We take the same gauge fixing fermion and the same boundary conditions as in the case of the Poisson sigma model in Section 12.1.3. Observables are correlation functions of $m+1$ vertex operators on the boundary. From the analysis of the moduli of insertion points of the observables, the observables on the boundary have the following form,

$$
\mathcal{O}_{x}\left(F_{0}, \ldots, F_{m}\right)=\int_{B_{m}} d^{m-1} t\left[F_{0}\left(\phi\left(t_{0}, \theta_{0}\right)\right) \cdots F_{m}\left(\phi\left(t_{m}, \theta_{m}\right)\right)\right]^{(m-1)} \delta_{x}(\phi(\infty)),
$$

where $t_{i}$ are the points on the boundary circle such that $1=t_{0}>t_{i}>\cdots>t_{m-1}>t_{m}=0$. $B_{m}$ is the space of the parameters $t_{i}$ and $[\cdots]^{(m-1)}$ denotes the order $\tau^{m-1}$-th term which is given by the integration over supercoordinates $\tau_{i}$. The map $U$ is given by

$$
U(\alpha)\left(F_{0} \otimes \cdots \otimes F_{m}\right)(x)=\int \mathcal{O}_{x}\left(F_{0}, \ldots, F_{m}\right) e^{\frac{i}{\hbar} S_{q}},
$$


where the path integral includes the $t_{i}$ integration over the moduli space $B_{m}$. We can obtain the $L_{\infty}$-morphism $U(\alpha)=\sum_{n=1}^{\infty} \frac{1}{n !} U_{n}\left(\alpha_{1}, \cdots, \alpha_{n}\right)$, where $U_{n}: \mathfrak{g}_{1}^{\otimes n} \longrightarrow \mathfrak{g}_{2}$. The concrete equation of $U$ is computed by the perturbative expansion of the path integral. The MC equation of the $L_{\infty}$-morphism is derived by using the WT identity induced from the quantum master equation as

$$
\begin{aligned}
& \sum_{\ell=0}^{n} \sum_{k=1}^{m-1} \sum_{i=0}^{m-k} \sum_{\sigma \in \mathfrak{S}_{l, n-l}} \epsilon(\sigma)(-1)^{k(i+1)}(-1)^{m} U_{l}\left(\alpha_{\sigma(1)}, \ldots, \alpha_{\sigma(l)}\right) \\
& \quad\left(F_{0} \otimes \cdots \otimes F_{i-1} \otimes U_{n-l}\left(\alpha_{\sigma(l+1)}, \ldots, \alpha_{\sigma(n)}\right)\left(F_{i} \otimes \cdots \otimes F_{i+k}\right) \otimes F_{i+k+1} \otimes \cdots \otimes F_{m}\right) \\
& =\sum_{i<j} \epsilon_{i j} U_{n-1}\left(\left[\alpha_{i}, \alpha_{j}\right], \alpha_{1}, \ldots, \widehat{\alpha}_{i}, \ldots, \widehat{\alpha}_{j}, \ldots, \alpha_{n}\right)\left(F_{0} \otimes \cdots \otimes F_{m}\right)
\end{aligned}
$$

where

$$
(i \hbar)^{n+m-1} U_{n}\left(\alpha_{1}, \ldots, \alpha_{n}\right)\left(F_{0} \otimes \cdots \otimes F_{m}\right)=\int \mathcal{O}_{x}\left(F_{0}, \ldots, F_{m}\right) e^{\frac{i}{\hbar} S_{0}} \frac{i}{\hbar} S_{\alpha_{1}} \cdots \frac{i}{\hbar} S_{\alpha_{n}}
$$

The map $U$ satisfying equation (12.163) is nothing but the $L_{\infty}$-morphism used in the proof of Theorem 12.8,

\section{Comments and Future Outlook}

The AKSZ construction is a clear method for the construction and analysis of topological field theories in any dimension. Although important aspects have been discussed here, we could not consider all topics related to AKSZ sigma models. We briefly list the subjects related to AKSZ sigma models that have not been discussed here.

The Poisson sigma model on a general Lie algebroid (the Lie algebroid Poisson sigma model) has been analyzed [20, 158, 145]. Several versions of TFTs with a generalized geometric structure have been constructed in two, three and higher dimensions [153, 154, 117, 79, 80, 40]. The Rozansky-Witten theory has been formulated by the AKSZ construction in three dimensions [118]. Open $p$-branes with worldvolume boundaries have been analyzed [116, 66]. A TFT with Dirac structure (a Dirac sigma model) has been formulated in Refs. [98, 100]. A three-dimensional version of the A-model has been proposed [139] and the relation between the doubled formalism and the AKSZ formalism has been analyzed [140]. A topological sigma model with a Nambu-Poisson structure (the Nambu-Poisson sigma model) has been 
constructed [25, 131]. The Poisson (and symplectic) reduction has been discussed in terms of the AKSZ approach [30, 43, 156, 157, 159, 17].

Many other geometric structures have been realized in the AKSZ construction [155, 120, 10, 86, 160].

General structures of this formulation and applications to various aspects of quantum field theories have been analyzed [12, 47, 48, 77, 99, 9, 18, 55]. The AKSZ construction on a discrete spacetime has been considered [19, 3]. Categorical and graded versions of bundles related to the AKSZ method, called derived geometry, have been formulated [115]. There are categorical and Chern-Weil formulations of the AKSZ construction [50]. The Wilson loop in the Chern-Simons theory has been formulated [2]. A current algebra theory based on the supergeometric AKSZ formulation has been constructed [78, 83]. The AKSZ sigma models have been applied to analyze T-duality and R-flux in string theory [113, 14]. The AKSZ formalism has been used in the construction of higher spin theories [23, 24, 22].

Supergeometry such as QP-manifolds is used to analyze the geometry of double field theory [46, 45, 60]. There are also recent papers that analyze AKSZ theories [1, 16, 85, 112].

The geometric structures of AKSZ theories have not yet been satisfactory analyzed. Many geometric structures have been realized by AKSZ sigma models, but there exist some structures for which the topological sigma model formulations have yet to be found. For example, the Nambu bracket itself, which appears in membrane theory, has not been constructed as a target space structure of an AKSZ type sigma model, although the Nambu-Poisson tensor has been realized by the AKSZ sigma model on a manifold with boundary [25]. Here, we did not fully discuss AKSZ theories on an open manifold, although we note that they are important and related to higher categories.

Many analyses of the quantization of AKSZ sigma models can be found in the literature [67, 21, 19, 119, 120, 86], but the analysis of the general AKSZ theory has not been completely understood. The gauge fixing procedure is complicated. It requires the BV formalism of component fields, since the gauge fixing is not formulated by superfields. Moreover, the moduli space of the observables in the path integral is not clear in more than two dimensions, and it is difficult to generalize the formality theorem.

Since gauge structures are algebroids, in general, their structures are highly nonlinear. Analysis of their structures, including their quantizations, is not so easy. For complete quan- 
tizations, we must solve the problem of globalization of algebroids to groupoids [34, 104]. Mathematical structures of algebroids and groupoids in general dimensions should be analyzed. Other important problems are the analysis of nonperturbative effects, such as instantons or monopoles.

AKSZ sigma models have not only reformulated topological invariants, but also led to the proposal of new topological or differential topological invariants. Thus, analysis of these models may solve problems, such as the classification of differential topological manifolds. It will also be important to clarify the relationship between the AKSZ formulation and the mathematical formulation of TFTs. [6]

In TFTs, mathematical and physical arguments are closely connected. AKSZ sigma models are rich in potential, and they lead to a deeper understanding of the relationship between mathematics and physics.

\section{Acknowledgments}

This lecture is partially based on my lecture series at Tohoku and Keio Universities, and I would like to thank them for their hospitality and discussions. The author would like to thank T. Asakawa, U. Carow-Watamura, T. Bessho, K. Koizumi, Y. Maeda, M. A. Heller, S. Sasa, M. Sato, K. Uchino, X. Xu and S. Watamura for valuable comments and discussions. He would like to thank Y. Maeda for encouraging me to write this lecture note, and thank U. Carow-Watamura, M. A. Heller and S. Watamura for careful reading of this manuscript. This work was supported by the Maskawa Institute, Kyoto Sangyo University and supported by the research promotion program grant at Ritsumeikan University.

\section{A Appendix: Formulas in Graded Differential Calculus}

We summarize the formulas of graded symplectic geometry. 


\section{A.1 Basic definitions}

Let $z$ be a local coordinate on a graded manifold $\mathcal{M}$. A differential on a function is defined as follows.

$$
d f(z)=d z^{a} \frac{\vec{\partial} f}{\partial z^{a}}
$$

A vector field $X$ is expanded using local coordinates, as follows.

$$
X=X^{a}(z) \frac{\vec{\partial}}{\partial z^{a}} .
$$

The interior product is defined using differentiation by the following graded vector field on $T[1] \mathcal{M}$

$$
\iota_{X}=(-1)^{|X|} X^{a}(z) \frac{\vec{\partial}}{\partial d z^{a}}
$$

where we define $\frac{\vec{\partial}}{\partial d z^{a}} d z^{b}=\delta^{b}{ }_{a}$. For a graded differential form $\alpha$, we denote by $|\alpha|$ the total degree (form degree plus degree by grading) of $\alpha$. Note that $|d|=1,\left|d z^{a}\right|=\left|z^{a}\right|+1$ and $\left|\iota_{X}\right|=|X|-1$. For vector fields, $X=X^{a}(z) \frac{\vec{\partial}}{\partial z^{a}}$ and $Y=Y^{a}(z) \frac{\vec{\partial}}{\partial z^{a}}$, the graded Lie bracket is

$$
[X, Y]=X^{a} \frac{\vec{\partial} Y^{b}}{\partial z^{a}} \frac{\vec{\partial}}{\partial z^{b}}-(-1)^{|X||Y|} Y^{a} \frac{\vec{\partial} X^{b}}{\partial z^{a}} \frac{\vec{\partial}}{\partial z^{b}}
$$

We obtain the following formula,

$$
X f=(-1)^{|X|} \iota_{X} d f=(-1)^{(|f|+1)|X|} d f(X),
$$

where

$$
d z^{a}\left(\frac{\vec{\partial}}{\partial z^{b}}\right)=\delta_{b}^{a}
$$

Proof We prove Eq. (A.168). Since $X f=X^{a}(z) \frac{\vec{\partial} f}{\partial z^{a}}$, we have

$$
(-1)^{|X|} \iota_{X} d f=(-1)^{|X|}(-1)^{|X|} X^{a}(z) \frac{\vec{\partial}}{\partial d z^{a}}\left(d z^{a} \frac{\vec{\partial} f}{\partial z^{a}}\right)
$$


Therefore,

$$
\begin{aligned}
d f(X) & =d z^{a} \frac{\vec{\partial} f}{\partial z^{a}}\left(X^{b}(z) \frac{\vec{\partial}}{\partial z^{b}}\right) \\
& =(-1)^{(|f|-|z|)|X|}\left[d z^{a}\left(X^{b}(z) \frac{\vec{\partial}}{\partial z^{b}}\right)\right] \frac{\vec{\partial} f}{\partial z^{a}} \\
& =(-1)^{(|f|-|z|)|X|}(-1)^{(|X|-|z|)(|z|+1)} X^{b}(z)\left[d z^{a}\left(\frac{\partial}{\partial z^{b}}\right)\right] \frac{\partial f}{\partial z^{a}} \\
& =(-1)^{(|f|+1)|X|} X^{a}(z) \frac{\vec{\partial} f}{\partial z^{a}}
\end{aligned}
$$

\section{A.2 Cartan formulas}

The Lie derivative is defined by

$$
L_{X}=\iota_{X} d-(-1)^{(|X|-1) \times 1} d \iota_{X}=\iota_{X} d+(-1)^{|X|} d \iota_{X} .
$$

Its degree is $\left|L_{X}\right|=|X|$.

Let $\alpha$ and $\beta$ be graded differential forms. We can show the following graded Cartan formulas,

$$
\begin{aligned}
& \alpha \wedge \beta=(-)^{|\alpha||\beta|} \beta \wedge \alpha, \\
& d(\alpha \wedge \beta)=d \alpha \wedge \beta+(-1)^{|\alpha|} \alpha \wedge d \beta, \\
& \iota_{X}(\alpha \wedge \beta)=\iota_{X} \alpha \wedge \beta+(-1)^{|\alpha|(|X|+1)} \alpha \wedge \iota_{X} \beta, \\
& L_{X}(\alpha \wedge \beta)=L_{X} \alpha \wedge \beta+(-1)^{|\alpha||X|} \alpha \wedge L_{X} \beta, \\
& L_{X} d=(-1)^{|X|} d L_{X}, \\
& \iota_{X} \iota_{Y}-(-1)^{(|X|-1)(|Y|-1)} \iota_{Y} \iota_{X}=0, \\
& L_{X} \iota_{Y}-(-1)^{|X|(|Y|-1)} \iota_{Y} L_{X}=\iota_{[X, Y]}, \\
& L_{X} L_{Y}-(-1)^{|X||Y|} L_{Y} L_{X}=L_{[X, Y]} .
\end{aligned}
$$

\section{A.3 Differential forms}

Let $\alpha=d z^{a_{1}} \wedge \cdots d z^{a_{m}} \alpha_{a_{1} \cdots a_{m}}(z)$ be an $m$-form on $\mathcal{M}$. The contraction of $\alpha(X,-, \cdots,-)$ with a vector field $X$ on $\mathcal{M}$ is

$$
\alpha(X,-, \cdots,-)=(-1)^{|X|(|\alpha|+1)} \iota_{X} \alpha(-, \cdots,-) .
$$




\section{Proof}

$$
\begin{aligned}
\alpha(X,-, \cdots,-)= & d z^{a_{1}} \wedge \cdots d z^{a_{m}} \alpha_{a_{1} \cdots a_{m}}(z)\left(X^{b} \frac{\vec{\partial}}{\partial z^{b}}\right) \\
= & (-1)^{|X|(|\alpha|-|z|-1)} d z^{a_{1}}\left(X^{b} \frac{\vec{\partial}}{\partial z^{b}}\right) d z^{a_{2}} \wedge \cdots d z^{a_{m}} \alpha_{a_{1} \cdots a_{m}}(z) \\
= & (-1)^{|X|(|\alpha|-|z|-1)}(-1)^{(|X|-|z|)(|z|+1)} \\
& \times X^{a_{1}} d z^{a_{2}} \wedge \cdots d z^{a_{m}} \alpha_{a_{1} \cdots a_{m}}(z) \\
= & (-1)^{|X||\alpha|} X^{a_{1}} d z^{a_{2}} \wedge \cdots d z^{a_{m}} \alpha_{a_{1} \cdots a_{m}}(z) \\
= & (-1)^{|X||\alpha|}(-1)^{|X|} \iota_{X} \alpha .
\end{aligned}
$$

By induction using Eq. (A.181), we obtain the following general formula,

$$
\begin{aligned}
& \alpha\left(X_{m}, X_{m-1}, \cdots, X_{1}\right)=-(-1)^{\sum_{i=1}^{m}\left|X_{i}\right|(|\alpha|+i)} \iota_{X_{m}} \cdots \iota_{X_{1}} \alpha, \\
& \alpha\left(X_{m}, \cdots, X_{j}, \cdots, X_{i}, \cdots X_{1}\right)=-(-1)^{\left|X_{i}\right|\left|X_{j}\right|} \alpha\left(X_{m}, \cdots, X_{i}, \cdots, X_{j}, \cdots X_{1}\right) .
\end{aligned}
$$

In particular, if $\alpha$ is a 2 -form, we obtain

$$
\alpha(X, Y)=-(-1)^{|X||Y|} \alpha(Y, X)
$$

\section{A.3.1 Exterior derivatives}

Recall the exterior derivative of a function was given by Eq. (A.168), i.e.

$$
d f(X)=(-1)^{|X|(|f|+1)} X f .
$$

Let $\alpha$ be a 1 -form on $\mathcal{M}$. Then, from the Cartan formulas, we obtain

$$
d \alpha\left(X_{1}, X_{2}\right)=(-1)^{\left|X_{1}\right||\alpha|} X_{1} \alpha\left(X_{2}\right)-(-1)^{\left|X_{2}\right||\alpha|}(-1)^{\left|X_{1}\right|\left|X_{2}\right|} X_{2} \alpha\left(X_{1}\right)-\alpha\left(\left[X_{1}, X_{2}\right]\right) .
$$

For a 2-form $\alpha$, the formula gives

$$
\begin{aligned}
d \alpha\left(X_{1}, X_{2}, X_{3}\right)= & (-1)^{\left|X_{1}\right|(|\alpha|+1)} X_{1} \alpha\left(X_{2}, X_{3}\right)-(-1)^{\left|X_{2}\right|(|\alpha|+1)}(-1)^{\left|X_{1}\right|\left|X_{2}\right|} X_{2} \alpha\left(X_{1}, X_{3}\right) \\
& +(-1)^{\left|X_{3}\right|(|\alpha|+1)}(-1)^{\left(\left|X_{1}\right|+\left|X_{2}\right|\right)\left|X_{3}\right|} X_{3} \alpha\left(X_{1}, X_{2}\right)-\alpha\left(\left[X_{1}, X_{2}\right], X_{3}\right) \\
& +(-1)^{\left|X_{2}\right|\left|X_{3}\right|} \alpha\left(\left[X_{1}, X_{3}\right], X_{2}\right)-(-1)^{\left|X_{1}\right|\left(\left|X_{2}\right|+\left|X_{3}\right|\right)} \alpha\left(\left[X_{2}, X_{3}\right], X_{1}\right)
\end{aligned}
$$


Let $\alpha=d z^{a_{1}} \wedge \cdots d z^{a_{m}} \alpha_{a_{1} \cdots a_{m}}(z)$ be an $m$-form on $\mathcal{M}$. Then, we can prove the following formula by induction,

$$
\begin{aligned}
d \alpha\left(X_{1}, X_{2}, \cdots, X_{m}\right)= & \sum_{i=1}^{m}(-1)^{i-1}(-1)^{\left|X_{i}\right|(|\alpha|+m)}(-1)^{\sum_{k=1}^{i-1}\left|X_{i}\right|\left|X_{k}\right|} X_{i} \alpha\left(X_{1}, \cdots, \hat{X}_{i}, \cdots, X_{m}\right) \\
& +\sum_{i<j}(-1)^{i+j}(-1)^{\sum_{k=1}^{i-1}\left|X_{i}\right|\left|X_{k}\right|+\sum_{l=1, l \neq j}^{j-1}\left|X_{j}\right|\left|X_{l}\right|} \\
& \times \alpha\left(\left[X_{i}, X_{j}\right], \cdots, \hat{X}_{i}, \cdots, \hat{X}_{j}, \cdots, X_{m}\right) .
\end{aligned}
$$

\section{A.4 Graded symplectic form and Poisson bracket}

Let $\omega$ be a symplectic form of degree $n$. Since $\omega$ is a 2-form, its total degree is $|\omega|=n+2$. Let $z=\left(q^{a}, p_{a}\right)$ be Darboux coordinates such that $|q|+|p|=n$. Then, we obtain

$$
\begin{aligned}
\omega & =(-1)^{|q|(|p|+1)} d q^{a} \wedge d p_{a}=(-1)^{n|q|} d q^{a} \wedge d p_{a} \\
& =(-1)^{n|q|}(-1)^{(|q|+1)(|p|+1)} d p_{a} \wedge d q^{a}=(-1)^{|p|+1} d p_{a} \wedge d q^{a} .
\end{aligned}
$$

The Liouville 1-form $\omega=-d \vartheta$ is then given by

$$
\begin{aligned}
\vartheta & =(-1)^{|p|} p_{a} d q^{a}=-(-1)^{n+1-|q|} p_{a} d q^{a}=(-1)^{|q||p|} d q^{a} p_{a} \\
& =-(-1)^{|q|(|p|+1)} q^{a} d p_{a}=-d p_{a} q^{a} .
\end{aligned}
$$

The Hamiltonian vector field $X_{f}$ of a function $f$ is defined by

$$
\iota_{X_{f}} \omega=-d f
$$

Its total degree is $\left|X_{f}\right|=|f|-n$. In order to obtain the Darboux coordinate expression of $X_{f}$, we take a local coordinate expression $X=X_{a} \frac{\vec{\partial}}{\partial p_{a}}+Y^{a} \frac{\vec{\partial}}{\partial q^{a}}$. Then we obtain

$$
\begin{aligned}
\iota_{X_{f}} \omega & =\left((-1)^{|X|+p} X_{a} \frac{\vec{\partial}}{\partial d p_{a}}+(-1)^{|X|+q} Y^{a} \frac{\vec{\partial}}{\partial d q^{a}}\right) \cdot\left((-1)^{n|q|} d q^{a} \wedge d p_{a}\right) \\
& =-d q^{a} \frac{\vec{\partial} f}{\partial q^{a}}-d p_{a} \frac{\vec{\partial} f}{\partial p_{a}} .
\end{aligned}
$$

By solving this equation, we finally obtain

$$
X_{f}=\frac{f \overleftarrow{\partial}}{\partial q^{a}} \frac{\vec{\partial}}{\partial p_{a}}-(-1)^{|q||p|} \frac{f \overleftarrow{\partial}}{\partial p_{a}} \frac{\vec{\partial}}{\partial q^{a}}
$$


Here, $\frac{f \overleftarrow{\partial}}{\partial q^{a}}=(-1)^{(|f|-q) q} \frac{\vec{\partial} f}{\partial q^{a}}$ is the right derivative.

The graded Poisson bracket is defined by

$$
\{f, g\}=X_{f} g=(-1)^{|f|+n} \iota_{X_{f}} d g=(-1)^{|f|+n+1} \iota_{X_{f}} \iota_{X_{g}} \omega .
$$

It satisfies

$$
\begin{aligned}
\{f, g\} & =-(-1)^{(|f|-n)(|g|-n)}\{g, f\}, \\
\{f, g h\} & =\{f, g\} h+(-1)^{(|f|-n)|g|} g\{f, h\}, \\
\{f,\{g, h\}\} & =\{\{f, g\}, h\}+(-1)^{(|f|-n)(|g|-n)}\{g,\{f, h\}\} .
\end{aligned}
$$

For the Darboux coordinates, we get the relations

$$
\left\{q^{a}, p_{b}\right\}=\delta_{b}^{a}, \quad\left\{p_{b}, q^{a}\right\}=-(-1)^{|q||p|} \delta_{b}^{a} .
$$

For the functions $f=f(q, p)$ and $g=g(q, p)$, the graded Poisson bracket is given by

$$
\{f, g\}=\frac{f \overleftarrow{\partial}}{\partial q^{a}} \frac{\vec{\partial} g}{\partial p_{a}}-(-1)^{|q||p|} \frac{f \overleftarrow{\partial}}{\partial p_{a}} \frac{\vec{\partial} g}{\partial q^{a}}
$$

$X$ is called a symplectic vector field if $L_{X} \omega=0$, i.e., $d \iota_{X} \omega=0$. Let $X, Y$ be symplectic vector fields. Then, $[X, Y]$ is the Hamiltonian vector field for $-(-1)^{|X|} \iota_{X} \iota_{Y} \omega$.

\section{Proof}

$$
\begin{aligned}
\iota_{[X, Y]} \omega & =\left(L_{X} \iota_{Y}-(-1)^{|X|(|Y|-1)} \iota_{Y} L_{X}\right) \omega=(-1)^{|X|} d \iota_{X} \iota_{Y} \omega \\
& =-d\left[-(-1)^{|X|} \iota_{X} \iota_{Y} \omega\right] .
\end{aligned}
$$

If $X=X_{f}, Y=X_{g}$ are Hamiltonian vector fields, then the following equation holds,

$$
\iota_{\left[X_{f}, X_{g}\right]} \omega=(-1)^{|f|+n} d \iota_{X_{f}} \iota_{X_{g}} \omega .
$$

Therefore, we get

$$
X_{\{f, g\}}=-\left[X_{f}, X_{g}\right]
$$

Since $\iota_{X_{f}} \iota_{X_{g}} \omega=-(-1)^{|f| n+|g|(n+1)} \omega\left(X_{g}, X_{f}\right)$, we easily obtain

$$
\begin{aligned}
\{f, g\} & =(-1)^{|f|+n+1} \iota_{X_{f}} \iota_{X_{g}} \omega \\
& =(-1)^{(|f|+|g|)(n+1)} \omega\left(X_{g}, X_{f}\right) \\
& =(-1)^{|f||g|+n+1} \omega\left(X_{f}, X_{g}\right) .
\end{aligned}
$$


We consider the $\operatorname{AKSZ}$ construction on $\operatorname{Map}(\mathcal{X}, \mathcal{M})$. Let $D$ be a differential on $\mathcal{X}$. It can be locally expressed as $D=\theta^{\mu} \frac{\partial}{\partial \sigma^{\mu}}$. We denote by $\hat{D}$ the vector field on $\operatorname{Map}(\mathcal{X}, \mathcal{M})$ of degree 1 which is induced by $D$. The following equation holds,

$$
\begin{aligned}
\left\{\iota_{\hat{D}} \mu_{*} \mathrm{ev}^{*} \vartheta, \mu_{*} \mathrm{ev}^{*} f\right\} & =-\iota_{\hat{D}} \mu_{*} \mathrm{ev}^{*} d f \\
& =\int d^{n+1} \sigma d^{n+1} \theta \boldsymbol{d} f(\sigma, \theta),
\end{aligned}
$$

for $f \in C^{\infty}(\mathcal{M})$.

Proof $S_{0}=\iota_{\hat{D}} \mu_{*} \mathrm{ev}^{*} \vartheta$ is a Hamiltonian for the vector field $\hat{D}$, i.e., $X_{S_{0}}=\hat{D}$. Therefore, we have

$$
\begin{aligned}
\left\{\iota_{\hat{D}} \mu_{*} \mathrm{ev}^{*} \vartheta, \mu_{*} \mathrm{ev}^{*} f\right\} & =\left\{S_{0}, \mu_{*} \mathrm{ev}^{*} f\right\} \\
& =(-1)^{\left|S_{0}\right|} \iota \hat{D}^{\iota} X_{\mu_{* \mathrm{ev}} f} \boldsymbol{\omega} \\
& =-\iota_{\hat{D}} \mu_{*} \mathrm{ev}^{*} d f .
\end{aligned}
$$

\section{References}

[1] K. B. Alkalaev and M. Grigoriev, "Frame-like Lagrangians and presymplectic AKSZ-type sigma models," Int. J. Mod. Phys. A 29 (2014) 18, 1450103 [arXiv:1312.5296 [hep-th]].

[2] A. Alekseev, Y. Barmaz and P. Mnev, "Chern-Simons Theory with Wilson Lines and Boundary in the BV-BFV Formalism," J. Geom. Phys. 67 (2013) 1 arXiv:1212.6256 [math-ph]].

[3] A. Alekseev and P. Mnev, "One-dimensional Chern-Simons theory," Commun. Math. Phys. 307 (2011) 185 arXiv:1005.2111 [hep-th]].

[4] A. Y. Alekseev, P. Schaller and T. Strobl, "The Topological G/G WZW model in the generalized momentum representation," Phys. Rev. D 52 (1995) 7146 arXiv:hep-th/9505012.

[5] M. Alexandrov, M. Kontsevich, A. Schwartz and O. Zaboronsky, "The Geometry of the master equation and topological quantum field theory," Int. J. Mod. Phys. A 12 (1997) 1405 arXiv:hep-th/9502010. 
[6] M. Atiyah, "Topological quantum field theories," Inst. Hautes Etudes Sci. Publ. Math. 68 (1989) 175.

[7] S. Axelrod and I. M. Singer, "Chern-Simons perturbation theory," arXiv:hep-th/9110056.

[8] G. Barnich, F. Brandt and M. Henneaux, "Local BRST cohomology in the antifield formalism. 1. General theorems," Commun. Math. Phys. 174 (1995) 57 arXiv:hep-th/9405109.

[9] G. Barnich and M. Grigoriev, "A Poincaré lemma for sigma models of AKSZ type," J. Geom. Phys. 61 (2011) 663 [arXiv:0905.0547 [math-ph]].

[10] G. Barnich and M. Grigoriev, "First order parent formulation for generic gauge field theories," JHEP 1101 (2011) 122 [arXiv:1009.0190 [hep-th]].

[11] G. Barnich and M. Henneaux, "Consistent couplings between fields with a gauge freedom and deformations of the master equation," Phys. Lett. B 311 (1993) 123 arXiv:hep-th/9304057.

[12] I. Batalin and R. Marnelius, "Superfield algorithms for topological field theories," In *Olshanetsky, M. (ed.) et al.: Multiple facets of quantization and supersymmetry* 233251 hep-th/0110140].

[13] I. A. Batalin and G. A. Vilkovisky, "Gauge Algebra And Quantization," Phys. Lett. B 102 (1981) 27; "Quantization Of Gauge Theories With Linearly Dependent Generators," Phys. Rev. D 28 (1983) 2567, [Erratum-ibid. D 30 (1984) 508].

[14] T. Bessho, M. A. Heller, N. Ikeda and S. Watamura, "Topological Membranes, Current Algebras and H-flux - R-flux Duality based on Courant Algebroids," arXiv:1511.03425 [hep-th].

[15] Y. Bi and Y. Sheng, "On higher analogues of Courant algebroids", Science China Mathematics 54 (2011) 437-447, arXiv:1003.1350[math.DG]

[16] G. Bonavolonta and A. Kotov, "Local BRST cohomology for AKSZ field theories: a global approach I," arXiv:1310.0245 [math-ph]. 
[17] F. Bonechi, A. Cabrera and M. Zabzine, "AKSZ construction from reduction data," arXiv:1204.2453 [hep-th].

[18] F. Bonechi, A. S. Cattaneo and P. Mnev, "The Poisson sigma model on closed surfaces," JHEP 1201 (2012) 099 arXiv:1110.4850 [hep-th]].

[19] F. Bonechi, P. Mnev and M. Zabzine, "Finite dimensional AKSZ-BV theories," Lett. Math. Phys. 94 (2010) 197 [arXiv:0903.0995 [hep-th]].

[20] F. Bonechi and M. Zabzine, "Lie algebroids, Lie groupoids and TFT," J. Geom. Phys. 57 (2007) 731 arXiv:math/0512245].

[21] F. Bonechi and M. Zabzine, "Poisson sigma model on the sphere," Commun. Math. Phys. 285 (2009) 1033 arXiv:0706.3164 [hep-th]].

[22] R. Bonezzi, N. Boulanger, E. Sezgin and P. Sundell, "An Action for Matter Coupled Higher Spin Gravity in Three Dimensions," arXiv:1512.02209 [hep-th].

[23] N. Boulanger and P. Sundell, "An action principle for Vasiliev's four-dimensional higherspin gravity," J. Phys. A 44 (2011) 495402 arXiv:1102.2219 [hep-th]].

[24] N. Boulanger, N. Colombo and P. Sundell, "A minimal BV action for Vasiliev's fourdimensional higher spin gravity," JHEP 1210 (2012) 043 [arXiv:1205.3339 [hep-th]].

[25] P. Bouwknegt and B. Jurco, "AKSZ construction of topological open p-brane action and Nambu brackets," Rev. Math. Phys. 25 (2013) 1330004 [arXiv:1110.0134 [math-ph]].

[26] I. Calvo, F. Falceto and D. Garcia-Alvarez, "Topological Poisson sigma models on Poisson lie groups," JHEP 0310 (2003) 033 arXiv:hep-th/0307178.

[27] C. Carmeli, L. Caston and R. Fioresi, "Mathematical Foundation of Supersymmetry," with an appendix with I. Dimitrov, EMS Ser. Lect. Math., European Math. Soc., Zurich 2011.

[28] U. Carow-Watamura, M. A. Heller, N. Ikeda, Y. Kaneko and S. Watamura, "Higher Gauge Theories from Lie n-algebras and Off-Shell Covariantization,” JHEP 1607 (2016) 125 arXiv:1606.03861 [hep-th]]. 
[29] A. S. Cattaneo, "Cabled Wilson loops in BF theories," J. Math. Phys. 37 (1996) 36843703. q-alg/9602015.

[30] A. S. Cattaneo, "Deformation quantization and reduction," math/0701378 [math-qa].

[31] A. S. Cattaneo, P. Cotta-Ramusino, A. Gamba, M. Martellini, "The Donaldson-Witten invariants in pure 4-D QCD with order and disorder 't Hooft - like operators," Phys. Lett. B355 (1995) 245-254. [hep-th/9502110].

[32] A. S. Cattaneo, P. Cotta-Ramusino and C. A. Rossi, "Loop observables for BF theories in any dimension and the cohomology of knots," Lett. Math. Phys. 51 (2000) 301, math.qa/0003073;

[33] A. S. Cattaneo and G. Felder, "A Path integral approach to the Kontsevich quantization formula," Commun. Math. Phys. 212 (2000) 591 arXiv:math/9902090.

[34] A. S. Cattaneo and G. Felder, "Poisson sigma models and symplectic groupoids," math/0003023 [math-sg].

[35] A. S. Cattaneo and G. Felder, "On the AKSZ formulation of the Poisson sigma model," Lett. Math. Phys. 56, 163 (2001) arXiv:math/0102108.

[36] A. S. Cattaneo and G. Felder, "Coisotropic submanifolds in Poisson geometry and branes in the Poisson sigma model," Lett. Math. Phys. 69 (2004) 157 math/0309180 [math-qa]].

[37] A. S. Cattaneo and G. Felder, "Effective Batalin-Vilkovisky theories, equivariant configuration spaces and cyclic chains," in Higher Structures in Geometry and Physics, Progress in Mathematics, 2011, Vol. 287 111-137, arXiv:0802.1706 [math-ph].

[38] A. Cattaneo, P. Mnev and N. Reshetikhin, "Classical BV theories on manifolds with boundaries," arXiv:1201.0290 [math-ph].

[39] A. S. Cattaneo, P. Mnev and N. Reshetikhin, "Classical and quantum Lagrangian field theories with boundary," PoS CORFU 2011 (2011) 044 [arXiv:1207.0239 [math-ph]].

[40] A. S. Cattaneo, J. Qiu and M. Zabzine, "2D and 3D topological field theories for generalized complex geometry," Adv. Theor. Math. Phys. 14 (2010) 695 arXiv:0911.0993 [hep-th]]. 
[41] A. S. Cattaneo and C. A. Rossi, "Higher dimensional BF theories in the BatalinVilkovisky formalism: the BV action and generalized Wilson loops," Commun. Math. Phys. 221 (2001) 591 arXiv:math/0010172.

[42] A. S. Cattaneo, F. Schätz, "Introduction to supergeometry," arXiv:1011.3401 [math$\mathrm{ph}]$.

[43] A. S. Cattaneo and M. Zambon, "Graded geometry and Poisson reduction," AIP Conf. Proc. 1093 (2009) 48.

[44] T. Courant. "Dirac manifolds," Trans. A. M. S. 319 (1990) 631.

[45] A. Deser and C. Sëmann, "Extended Riemannian Geometry I: Local Double Field Theory," arXiv:1611.02772 [hep-th].

[46] A. Deser and J. Stasheff, "Even symplectic supermanifolds and double field theory," Commun. Math. Phys. 339 (2015) 3, 1003 arXiv:1406.3601 [math-ph]].

[47] L. Edgren and N. Sandström, "First order gauge field theories from a superfield formulation," JHEP 0209 (2002) 036 hep-th/0205273.

[48] L. Edgren and N. Sandström, "Superfield algorithm for higher order gauge field theories," JHEP 0401 (2004) 006 hep-th/0306175.

[49] F. Falceto and K. Gawedzki, "Boundary G/G theory and topological Poisson-Lie sigma model," Lett. Math. Phys. 59 (2002) 61 arXiv:hep-th/0108206.

[50] D. Fiorenza, C. L. Rogers and U. Schreiber, "A Higher Chern-Weil derivation of AKSZ $\sigma$-models," arXiv:1108.4378 [math-ph].

[51] R. Fulp, T. Lada and J. Stasheff, "Sh-Lie algebras Induced by Gauge Transformations," math.qa/0012106.

[52] J. Gomis, J. Paris and S. Samuel, "Antibracket, antifields and gauge theory quantization," Phys. Rept. 259 (1995) 1 arXiv:hep-th/9412228.

[53] J. Grabowski, D. Khudaverdyan, N. Poncin, "Loday algebroids and their supergeometric interpretation," arXiv:1103.5852 [math.DG]. 
[54] J. Grabowski, M. Rotkiewicz, "Higher vector bundles and multi-graded symplectic manifolds," J. Geom. Phys. 59 (2009), 1285-1305. arXiv:math/0702772.

[55] M. Grigoriev, "Parent formulations, frame-like Lagrangians, and generalized auxiliary fields," arXiv:1204.1793 [hep-th].

[56] M. Grützmann, "H-twisted Lie algebroid," J. Geom. Phys. 61 (2011), 476, arXiv:1005.5680.

[57] M. Gualtieri, "Generalized complex geometry," arXiv:math/0401221.

[58] Y. Hagiwara, "Nambu-Dirac manifolds," J. Phys. A: Math. Gen. 35 (2002) 1263.

[59] M. Hansen and T. Strobl, "First Class Constrained Systems and Twisting of Courant Algebroids by a Closed 4-form," arXiv:0904.0711 [hep-th].

[60] M. A. Heller, N. Ikeda and S. Watamura, "Unified picture of non-geometric fluxes and T-duality in double field theory via graded symplectic manifolds," arXiv:1611.08346] [hepth].

[61] M. Henneaux, "Lectures on the Antifield-BRST Formalism for Gauge Theories," Nucl. Phys. Proc. Suppl. 18A (1990) 47.

[62] M. Henneaux, "Consistent interactions between gauge fields: The Cohomological approach," Contemp. Math. 219 (1998) 93 arXiv:hep-th/9712226.

[63] M. Henneaux and C. Teitelboim, "Quantization of gauge systems," Princeton, USA: Univ. Pr. (1992) $520 p$

[64] N. Hitchin, "Generalized Calabi-Yau manifolds," Quart. J. Math. Oxford Ser. 54 (2003) 281 arXiv:math/0209099.

[65] C. Hofman, "On the open-closed B-model," JHEP 0311 (2003) 069 arXiv:hep-th/0204157.

[66] C. Hofman and J. S. Park, "Topological open membranes," hep-th/0209148.

[67] C. Hofman and J. S. Park, "BV quantization of topological open membranes," Commun. Math. Phys. 249 (2004) 249 arXiv:hep-th/0209214. 
[68] G. T. Horowitz, "Exactly Soluble Diffeomorphism Invariant Theories," Commun. Math. Phys. 125 (1989) 417. doi:10.1007/BF01218410

[69] N. Ikeda, "Two-dimensional gravity and nonlinear gauge theory," Annals Phys. 235 (1994) 435 arXiv:hep-th/9312059].

[70] N. Ikeda, "A deformation of three dimensional BF theory," JHEP 0011 (2000) 009, hep-th/0010096.

[71] N. Ikeda, "Deformation of BF theories, topological open membrane and a generalization of the star deformation," JHEP 0107 (2001) 037, hep-th/0105286.

[72] N. Ikeda, "Chern-Simons gauge theory coupled with BF theory," Int. J. Mod. Phys. A 18 (2003) 2689 arXiv:hep-th/0203043.

[73] N. Ikeda, "Topological field theories and geometry of Batalin-Vilkovisky algebras," JHEP 0210 (2002) 076 hep-th/0209042.

[74] N. Ikeda, "Deformation of Batalin-Vilkovisky Structures," arXiv:math.sg/0604157.

[75] N. Ikeda, "Donaldson Invariants and Their Generalizations from AKSZ Topological Field Theories," arXiv:1104.2100 [hep-th].

[76] N. Ikeda and K. I. Izawa, "General form of dilaton gravity and nonlinear gauge theory," Prog. Theor. Phys. 90 (1993) 237 arXiv:hep-th/9304012.

[77] N. Ikeda and K. I. Izawa, "Dimensional reduction of nonlinear gauge theories," JHEP 0409 (2004) 030 hep-th/0407243.

[78] N. Ikeda and K. Koizumi, "Current Algebras and QP Manifolds," Int. J. Geom. Meth. Mod. Phys. 10 (2013) 1350024 arXiv:1108.0473 [hep-th]].

[79] N. Ikeda and T. Tokunaga, "Topological Membranes with 3-Form H Flux on Generalized Geometries," Adv. Theor. Math. Phys. 12 (2008) 1259 hep-th/0609098.

[80] N. Ikeda and T. Tokunaga, "An Alternative Topological Field Theory of Generalized Complex Geometry," JHEP 0709 (2007) 009 [arXiv:0704.1015 [hep-th]]. 
[81] N. Ikeda and K. Uchino, "QP-Structures of Degree 3 and 4D Topological Field Theory," Comm. Math. Phys. 303 (2011) 317-330, arXiv:1004.0601 [hep-th].

[82] N. Ikeda and X. Xu, 'Canonical Functions and Differential Graded Symplectic Pairs in Supergeometry and AKSZ Sigma Models with Boundary," J. of Math. Phys. 55 (2014) 113505, arXiv:1301.4805 [math.SG].

[83] N. Ikeda and X. Xu, "Current Algebras from DG Symplectic Pairs in Supergeometry," arXiv:1308.0100 [math-ph].

[84] K. I. Izawa, "On nonlinear gauge theory from a deformation theory perspective," Prog. Theor. Phys. 103 (2000) 225 [arXiv:hep-th/9910133].

[85] T. Johnson-Freyd, "Poisson AKSZ theories and their quantizations," Proc. Symp. Pure Math. 88 (2014) 291 arXiv:1307.5812 [math-ph]].

[86] J. Kallen, J. Qiu and M. Zabzine, "Equivariant Rozansky-Witten classes and TFTs," arXiv:1011.2101 [hep-th].

[87] H. O. M. Khudaverdian, "Semidensities on odd symplectic supermanifolds," Commun. Math. Phys. 247 (2004) 353, arXiv:math.DG/0012256.

[88] H. M. Khudaverdian and T. Voronov, "On odd Laplace operators," Lett. Math. Phys. 62 (2002) 127 arXiv:math/0205202].

[89] H. M. Khudaverdian and T. Voronov, "On odd Laplace operators. II," arXiv:math/0212311.

[90] L. Kjeseth, "Homotopy Rinehart cohomology of homotopy Lie-Rinehart pairs," Homology, Homotopy and Application 3 (2001) 139.

[91] C. Klimcik and T. Strobl, "WZW-Poisson manifolds," J. Geom. Phys. 43 (2002) 341 [arXiv:math/0104189].

[92] M. Kontsevich, Lectures at IHES. Fall 1995.

[93] M. Kontsevich, "Deformation quantization of Poisson manifolds," Lett. Math. Phys. 66 (2003) 157 [arXiv:q-alg/9709040]. 
[94] Y. Kosmann-Schwarzbach, J. Monterde, "Divergence operators and odd Poisson brackets, " Ann. Inst. Fourier (Grenoble) 52 (2002) 419, math.QA/0002209.

[95] Y. Kosmann-Schwarzbach, "Derived brackets," Lett. Math. Phys. 69, 61 (2004), arXiv:math.dg/0312524.

[96] Y. Kosmann-Schwarzbach. "Quasi-, twisted, and all that... in Poisson geometry and Lie algebroid theory." The Breadth of Symplectic and Poisson Geometry, Festschrift in honor of Alan Weinstein, Progress in Mathematics. 232. (2005). 363-389, math.SG/0310359.

[97] Y. Kosmann-Schwarzbach, "Poisson and symplectic functions in Lie algebroid theory," Higher Structures in Geometry and Physics, in honor of Murray Gerstenhaber and Jim Stasheff, eds. Alberto Cattaneo, Antony Giaquinto and Ping Xu, Progress in Mathematics 287, Birkhauser, 2011, 243-268, arXiv:0711.2043.

[98] A. Kotov, P. Schaller and T. Strobl, "Dirac sigma models," Commun. Math. Phys. 260 (2005) 455 arXiv:hep-th/0411112.

[99] A. Kotov and T. Strobl, "Characteristic classes associated to Q-bundles," arXiv:0711.4106 [math.DG].

[100] A. Kotov and T. Strobl, "Generalizing Geometry - Algebroids and Sigma Models," arXiv:1004.0632 [hep-th].

[101] T. Lada and M. Markl, "Strongly homotopy Lie algebras," arXiv:hep-th/9406095.

[102] T. Lada and J. Stasheff, "Introduction to SH Lie algebras for physicists," Int. J. Theor. Phys. 32 (1993) 1087, hep-th/9209099.

[103] A. M. Levin and M. A. Olshanetsky, "Hamiltonian algebroid symmetries in W-gravity and Poisson sigma-model," hep-th/0010043.

[104] D. Li-Bland, P. Ševera "Integration of Exact Courant Algebroids," arXiv:1101.3996 [math.DG].

[105] Z.-J. Liu, A. Weinstein and P. Xu, "Manin triples for Lie bialgebroids," J. Diff. Geom. 45 (1997), 547-574. 
[106] J-L. Loday. "Dialgebras." Lecture Notes in Mathematics, 1763. Springer-Verlag, Berlin, (2001), 7-66.

[107] J-L. Loday and T. Pirashvili. "Universal enveloping algebras of Leibniz algebras and (co)homology," Math. Ann. 296 (1993), 139-158.

[108] J. Luire. "On the Classification of Topological Field Theories." Current Developments in Mathematics Volume 2008 (2009), 129-280, arXiv:0905.0465.

[109] K. Mackenzie, "Lie Groupoids and Lie Algebroids in Differential Geometry," LMS Lecture Note Series 124, Cambridge U. Press, 1987.

[110] Y. Maeda, H. Kajiura, "String Theory and Deformation Quantization"(Japanese), Sugaku expositions 20. (2007), 191-214.

[111] Y. Manin, "Gauge field theory and complex geometry," Grundlehren der Mathematischen Wissenschaften, 289. Springer-Verlag, Berlin, 1997.

[112] P. Mnev, "A construction of observables for AKSZ sigma models," arXiv:1212.5751 [math-ph].

[113] D. Mylonas, P. Schupp and R. J. Szabo, "Membrane Sigma-Models and Quantization of Non-Geometric Flux Backgrounds," JHEP 1209 (2012) 012 [arXiv:1207.0926 [hep-th]].

[114] M. A. Olshanetsky, "Lie algebroids as gauge symmetries in topological field theories," hep-th/0201164.

[115] T. Pantev, B. Toen, M. Vaquie and G. Vezzosi, "Shifted symplectic structures," arXiv:1111.3209 [math.AG]

[116] J. S. Park, "Topological open p-branes," in: Symplectic Geometry and Mirror Symmetry, eds. K. Fukaya, Y.-G. Oh, K. Ono and G. Tian (World Scientific, 2001) 311-384 hep-th/0012141.

[117] V. Pestun, "Topological strings in generalized complex space," Adv. Theor. Math. Phys. 11 (2007) 399 [hep-th/0603145]. 
[118] J. Qiu and M. Zabzine, "On the AKSZ formulation of the Rozansky-Witten theory and beyond," JHEP 0909 (2009) 024 [arXiv:0906.3167 [hep-th]].

[119] J. Qiu and M. Zabzine, "Odd Chern-Simons Theory, Lie Algebra Cohomology Commun. Math. Phys. 300 (2010) 789-833. arXiv:0912.1243 [hep-th]].

[120] J. Qiu and M. Zabzine, "Knot Invariants and New Weight Systems from General 3D TFTs," J. Geom. Phys. 62 (2012) 242 [arXiv:1006.1240 [hep-th]].

[121] J. Qiu and M. Zabzine, "Introduction to Graded Geometry, Batalin-Vilkovisky Formalism and their Applications," arXiv:1105.2680 [math.QA].

[122] D. Roytenberg, "Courant algebroids, derived brackets and even symplectic supermanifolds," math.DG/9910078.

[123] D. Roytenberg, "On the structure of graded symplectic supermanifolds and Courant algebroids," Quantization, Poisson Brackets and Beyond, Theodore Voronov (ed.), Contemp. Math., Vol. 315, Amer. Math. Soc., Providence, RI, 2002, math.sg/0203110.

[124] D. Roytenberg, "AKSZ-BV formalism and Courant algebroid-induced topological field theories," Lett. Math. Phys. 79 (2007) 143 arXiv:hep-th/0608150.

[125] D. Roytenberg, "On weak Lie 2-algebras," In XXVI Workshop on Geometrical Methods in Physics, volume 956 of AIP Conference Proceedings, Piotr Kielanowski et al (eds). American Institute of Physics, Melville, NY, 2007, arXiv:0712.3461[math.QA].

[126] D. Roytenberg and A. Weinstein "Courant algebroids and strongly homotopy Lie algebras." Lett. in Math. Phys. 46, 81 (1998) arXiv:math.QA/9802118.

[127] L. Rozansky and E. Witten, "HyperKähler geometry and invariants of three manifolds," Selecta Math. 3 (1997) 401 doi:10.1007/s000290050016 hep-th/9612216.

[128] P. Schaller and T. Strobl, "Poisson structure induced (topological) field theories," Mod. Phys. Lett. A 9 (1994) 3129 arXiv:hep-th/9405110.

[129] P. Schaller and T. Strobl, "Poisson sigma models: A Generalization of 2-d gravity Yang-Mills systems," In *Dubna 1994, Finite dimensional integrable systems* 181-190 hep-th/9411163. 
[130] M. Schlessinger, J. Stasheff, "The Lie algebra structure on tangent cohomology and deformation theory," J. Pure Appl. Algebra 89 (1993) 231.

[131] P. Schupp and B. Jurčo, "Nambu Sigma Model and Branes," Rev. Math. Phys. 25 (2013) 1330004, arXiv:1205.2595 [hep-th].

[132] A. Schwarz, "Geometry of Batalin-Vilkovisky quantization," Commun. Math. Phys. 155 (1993) 249, hep-th/9205088.

[133] A. S. Schwarz, "Semiclassical approximation in Batalin-Vilkovisky formalism," Commun. Math. Phys. 158 (1993) 373 arXiv:hep-th/9210115.

[134] T. Schwarzweller, "The Poisson-sigma model: A non-linear gauge theory," Contributed to 3rd International Conference on Geometry, Integrability and Quantization, St. Constantine, Bulgaria, 14-23 Jun 2001, hep-th/0111141.

[135] N. Seiberg and E. Witten, "String theory and noncommutative geometry," JHEP 9909 (1999) 032 hep-th/9908142.

[136] P. Ševera, "Some title containing the words "homotopy" and "symplectic", e.g. this one," Travaux Math. 16 (2005) 121-137, arXiv:math/0105080.

[137] P. Ševera and A. Weinstein, "Poisson geometry with a 3-form background," Prog. Theor. Phys. Suppl. 144 (2001) 145 arXiv:math/0107133].

[138] J. Stasheff, "Deformation Theory and the Batalin-Vilkovisky Master Equation," q-alg/9702012.

[139] V. Stojevic, "Topological A-Type Models with Flux," JHEP 0805 (2008) 023 arXiv:0801.1160 [hep-th]].

[140] V. Stojevic, "Doubled Formalism, Complexification and Topological Sigma-Models," arXiv:0809.4034 [hep-th].

[141] Y. Terashima, "On Poisson functions," J. Sympl. Geom., 6(2008) 1-7.

[142] The author would like to thank to K .Uchino for discussions. 
[143] I. Vaisman, "Lectures on the Geometry of Poisson Manifolds," Progress in Math., vol. 118, Birkhauser Verlag, Basel, 1994.

[144] V. S. Varadarajan, "Supersymmetry for mathematicians: an introduction," Courant Lecture Notes Series, AMS, New York, 2004.

[145] A. Q. Velez, "Boundary coupling of Lie algebroid Poisson sigma models and representations up to homotopy," Lett. Math. Phys. 102 (2012) 31 arXiv:1108.6225 [math-ph]].

[146] J. Vey, "Déformation du crochet de Poisson sur une variété symplectique," Comment. Math. Helvet. 50 (1975) 421-454.

[147] T.Voronov, "Vector fields on mapping spaces and a converse to the AKSZ construction," arXiv:1211.6319 [math-ph].

[148] A. Wade, "Nambu-Dirac Structures on Lie Algebroids," Lett. Math. Phys. 61 (2002) 85-99 arXiv:math.SG/0204310.

[149] E. Witten, "Topological Quantum Field Theory," Commun. Math. Phys. 117 (1988) 353.

[150] E. Witten, "Topological Sigma Models," Commun. Math. Phys. 118 (1988) 411.

[151] E. Witten, "Mirror manifolds and topological field theory," In *Yau, S.T. (ed.): Mirror symmetry I* 121-160 hep-th/9112056.

[152] M. Zambon, "L-infinity algebras and higher analogues of Dirac structures and Courant algebroids," J. Symp .Geom. 10 (2012) 563 arXiv:1003.1004 [math.SG].

[153] R. Zucchini, "A Sigma model field theoretic realization of Hitchin's generalized complex geometry," JHEP 0411 (2004) 045 hep-th/0409181.

[154] R. Zucchini, "Generalized complex geometry, generalized branes and the Hitchin sigma model," JHEP 0503 (2005) 022 hep-th/0501062.

[155] R. Zucchini, "A Topological sigma model of Bikaehler geometry," JHEP 0601 (2006) 041 hep-th/0511144. 
[156] R. Zucchini, "The Hitchin Model, Poisson-quasi-Nijenhuis Geometry and Symmetry Reduction," JHEP 0710 (2007) 075 [arXiv:0706.1289 [hep-th]].

[157] R. Zucchini, "Gauging the Poisson sigma model," JHEP 0805 (2008) 018 arXiv:0801.0655 [hep-th]].

[158] R. Zucchini, "The Lie algebroid Poisson sigma model," JHEP 0812 (2008) 062 arXiv:0810.3300 [math-ph]].

[159] R. Zucchini, "The Gauging of BV algebras," J. Geom. Phys. 60 (2010) 1860 arXiv:1001.0219 [hep-th]].

[160] R. Zucchini, "AKSZ models of semistrict higher gauge theory," JHEP 1303 (2013) 014 arXiv:1112.2819 [hep-th]]. 